\title{
The physics of premelted ice and its geophysical consequences
}

\author{
J. G. Dash \\ Department of Physics, University of Washington, Seattle, Washington 98195-1560, USA
}

A. W. Rempel

Department of Geological Sciences, University of Oregon, Eugene, Oregon 97403, USA

\section{J. S. Wettlaufer}

Departments of Geology \& Geophysics and Physics, Yale University, New Haven, Connecticut 06520-8109, USA

(Published 12 July 2006)

\begin{abstract}
The surface of ice exhibits the swath of phase-transition phenomena common to all materials and as such it acts as an ideal test bed of both theory and experiment. It is readily available, transparent, optically birefringent, and probing it in the laboratory does not require cryogenics or ultrahigh vacuum apparatus. Systematic study reveals the range of critical phenomena, equilibrium and nonequilibrium phase-transitions, and, most relevant to this review, premelting, that are traditionally studied in more simply bound solids. While this makes investigation of ice as a material appealing from the perspective of the physicist, its ubiquity and importance in the natural environment also make ice compelling to a broad range of disciplines in the Earth and planetary sciences. In this review we describe the physics of the premelting of ice and its relationship with the behavior of other materials more familiar to the condensed-matter community. A number of the many tendrils of the basic phenomena as they play out on land, in the oceans, and throughout the atmosphere and biosphere are developed.
\end{abstract}

DOI: 10.1103/RevModPhys.78.695

\section{CONTENTS}

I. Overview: Liquid Films on Premelted Ice

A. Accounts and speculations on snow crystals and ice growth

696

B. Slippery if wet

C. Faraday, ice, and surface melting

D. Background: Relevant phenomena

1. Adsorption and wetting

2. Porous media

3. Premelting and phase change

4. Crystal shapes and roughening

II. Theory

A. Thermodynamics of premelting

B. Lifshitz theory of ice premelting

1. Pure ice at the vapor surface; incomplete surface melting

2. Pure ice at chemically inert walls: Interfacial melting

3. Grain-boundary melting in pure ice

C. Effects of nonideality

1. Nonplanar substrates

2. Internally disordered solids

3. Impurities

4. Summary, basic consequences, and caveats regarding impurity effects

D. Dynamical effects

1. Thermomechanical pressure

2. Flow by thermomolecular pressure

III. Computer Studies of Ice Premelting

IV. Laboratory Studies of Premelted Ice
PACS number(s): 64.70.Dv, 68.08.Bc, 92.40.Vq

A. Vapor surface of ice: Surface melting 715

B. Interfaces with solid substrates: Interfacial melting 716

C. Interfaces between two crystals: Grain-boundary melting

D. Probing ice in porous media $\quad 717$

E. Crystal growth 718

V. Environmental Phenomena $\quad 719$

A. Ice on land 719

1. Frost heave 719

2. Ice and weathering 722

3. Glacier motion 722

4. Veins and nodes in polycrystalline ice 724

5. Ice core climate proxies 724

6. Metamorphosis of snow 726

B. Ice on the oceans $\quad 727$

C. Ice in the atmosphere $\quad 728$

1. Ozone chemistry on polar stratospheric cloud particles

2. Electrical charging in thunderstorms 729

a. Inductive charging 729

b. Freezing potentials 729

c. Thermoelectricity 729

d. Contact potentials 729

e. Charging involving surface melting 729

f. Collisional charging and surface disorder $\quad 730$

g. Charging involving particle shape 731

h. Charging involving chemical impurities 732

i. Relationship to field studies 732

D. Extraterrestrial ice 732

VI. Interfacial Melting Sank an Aircraft Carrier 733

VII. Summary 734 
Acknowledgments

References

734

734

\section{OVERVIEW: LIQUID FILMS ON PREMELTED ICE}

The premelted liquid layers on ice are unique aqueous films. Borne by ice as both substrate and source, these films exist over a wide range of environments, from the depths of glaciers to stratospheric ice clouds. Their sensitivity to temperature, contaminants, and crystalline disorder makes them active participants with external conditions; they both influence and respond to their environments. They control the growth rates and shapes of snowflakes and the sintering of snowfields, and their directed transport of impurities along grain boundaries makes them major players in the dynamics of ice sheets. During collisions between ice particles and hail in thunderstorms the liquid films transport the electrical charges that power the lightning. Premelted films exist in all classes of solids, where they facilitate crystal growth from vapor and the coarsening of polycrystals. They are catalysts of crystallization, and they ease the transformation from solid to liquid, by removing the nucleation barrier to melting.

We begin with a historical survey; observations of snowflakes and speculations on the origins of their remarkable symmetry, and a review of the growth mechanism, which may depend, in most environments, on the existence of unfrozen surface layers. The earliest ideas on liquid surface layers were of a supposedly unique phenomenon of ice, but much later it was understood that premelting is common to all solids. Liquidlike layers exist, at equilibrium, on the "wrong" side of the bulk phase boundary, at interfaces between solids and vapor or at foreign substrates, and between grains of the same substance.

The general theory of premelting is described in detail, beginning with the thermodynamics of pure, wellordered materials. It is shown that the thickness of the liquid film and its temperature dependence are controlled by the fundamental interactions of the material and its substrate. Dynamic effects result from temperature gradients, producing important transport and pressure changes. The influence of various perturbations on the static and dynamic properties are studied; impurities and crystalline disorder extend the range of premelting, and contribute to dynamical phenomena. Ionizing impurities are especially complex, due to the combination of chemical and electrical effects. We also review the theory of premelting of materials governed by dispersion forces, and its application to the premelting of ice, which has been successful in describing the incomplete surface melting of pure ice at vapor interfaces.

An extended account is given of laboratory results for the premelting of ice at various interfaces, for it has been examined with a wide variety of techniques by many investigators. A considerable amount of disagreement exists among the results, which we conclude testifies to the great sensitivity to impurities and other perturbations. The experiments are discussed, with a view toward determining a set of reliable coefficients.

The environmental effects of premelted layers on ice form the last major section of the review. Many play significant roles in geophysical and in atmospheric phenomena. The review describes how the large-scale phenomena can depend essentially on the fundamental properties of premelting. These interactions are for the most part still under active investigation, and they continue to offer challenging fields for further research.

\section{A. Accounts and speculations on snow crystals and ice growth}

The most obvious and yet most compelling feature of crystalline matter is its shape. Commonplace and technical admiration of the form taken by a snowflake needs to be informed by the fact that what we see is typically not an equilibrium shape, but rather a form resulting from a disequilibrium process and subsequent isolation from efficient kinetic pathways that allow the material to equilibrate. Historically, our understanding of the relation between growth and equilibrium shapes emerges out of basic questions of geometry and the desire to control matter. At a sufficiently basic level the general issues are entirely independent of the material under consideration, but both technical and aesthetic considerations draw our attention to specific substances. Here, the latter dominate and we delve into these phenomenon as they bear on ice.

Ice dominates the crystal growth phenomena we observe on the surface of the earth and in the atmosphere. Most of the observed patterns arise out of the uncontrollable influence that the natural environment renders on growth forms (Wettlaufer, 2001; Adam, 2005). Modern research into the mechanisms underlying pattern formation in snowflakes is traced to the experiments of $\mathrm{Na}$ kaya (Nakaya, 1954; Furukawa, 1997) in which the first systematic laboratory studies correlated growth conditions with crystal shapes. As we shall see, most of the surface-specific structural phase transitions that occur in other materials are observed to occur in ice, where, among a plethora of other things, they influence equilibrium and growth forms. However, as opposed to many engineered materials, ice exists in the terrestrial environment at temperatures relatively close to its melting point. Moreover, through their influence on the interfacial structure, these surface phase transitions can control the adsorption potential, growth shapes, and surface transport properties of ice. Furthermore, because surface phase transitions occur in most materials, what we learn in ice is broadly relevant in condensed-matter science.

During the winter of 1635 Descartes made strikingly detailed observations of snow and ice that highlight the role of ice surfaces in growth, melting, and adhesive behavior. Some of these observations were translated from French by Sir Charles Frank (1974):

"But what astonished me the most was that among the grains that fell last night I noticed some which 
had around them six little teeth, like clock makers' wheels...but so perfectly formed in hexagones, and of which the six sides were so straight, and the six arms so equal, that it is impossible for man to make anything so exact."

"I was not surprised, either, to see often two stars of unequal size joined together...I judged that the cause was that the heat, having been stronger around the small one than the other, had to a greater extent melted and blunted its points."

Several centuries later, in a series of investigations in which he questioned the role of the surface in the slipperiness of ice, Michael Faraday initiated thoughts on the essential differences of the surface from the bulk (Faraday, 1933) (see Sec. I.C). Today we continue to explore, unravel, and marvel at the mysteries exposed by Descartes.

\section{B. Slippery if wet}

Ever since prehistoric times, snow and ice have enabled people to travel great distances; for hunting, migrating, and commerce between settlements. Ground freezing and snowfalls were welcomed after summer rains had turned trails and roads into quagmires. Today, we enjoy snow and ice for winter sports, while they are nuisances or worse for road travel. Easy sliding is both the boon and the bane.

The slipperiness of snow and ice is one of their most dominant and familiar features, but not if the temperature is very low. Here are two observations on polar travel. First, Fridtjof Nansen (1994), crossing Greenland:

"The severe cold we experienced made things, in this respect, unusually bad; the snow, as we were fond of saying, was as heavy as sand to pull upon."

And Donald MacMillan (1925), living among Eskimos in Alaska:

"At 36 below zero, the sledges (with steel runners) dragged hard over young ice covered with an inch of granular snow, sand could hardly have been worse."

Temperature is only one of the significant variables, another is pressure. In the 19th century James Thomson (1849), noting that the density of ice is lower than that of water, predicted that therefore ice would melt under pressure. His brother William, who was later raised to the peerage as Lord Kelvin (Thomson, 1861), applied the pressure melting mechanism to explain Michael Faraday's observation of regelation (Faraday, 1850), the welding of two blocks of ice, after contact pressure was relieved. Several years later, John Joly suggested that pressure melting could also be a simple explanation of why ice is slippery; the pressure applied by the ice skate melts the ice below it, and the water film acts as a lubricant (Joly, 1887). However, Joly also warned that the mechanism had limitations; it could not be effective for even the bite of a hollow ground blade if the ice is colder than a few degrees below $0{ }^{\circ} \mathrm{C}$. Pressure melting became the standard textbook explanation, and it has been propagated through generations of students since then, although without Joly's warning. Indeed, since the slope of the ice/water phase boundary is over $130 \mathrm{bars} / \mathrm{K}$, it cannot explain the common hazard of walking on ice, if the temperature is lower than $a$ few $m K$ below $0{ }^{\circ} \mathrm{C}$. But ice is slippery even at temperatures well below the freezing point, so something else besides pressure melting has to be operating. In fact there are two other mechanisms: surface melting and frictional melting.

Surface and interfacial premelting can produce liquidlike layers at temperatures as low as a few tens of degrees below zero, especially if there are appreciable surface impurities (see Sec II.C.3). However, the liquid films stemming from premelting are too thin to account for the very low friction coefficients that occur in certain cases; for example, in speed skating a value as low as 0.005 at $-17^{\circ} \mathrm{C}$ has been measured (De Koning, 1992). In speed skating and in many more common circumstances the dominant lubrication is meltwater, produced by frictional heating. This process was first suggested and tested in a laboratory study by Bowden and Hughes (1939). Many subsequent studies have confirmed it to be the controlling mechanism of ice friction over a wide range of temperatures, sliding speeds, and materials (Bowden, 1953; Jellinek, 1967; Evans et al., 1976; Barer et al., 1980; Colbeck, 1988, 1992; Colbeck and Warren, 1991; Bluhm et al., 2000; Liang et al., 2003, 2005). Samuel Colbeck has shown that snow friction is much more complicated because it also involves the work of compression and plowing (Colbeck, 1988, 1992; Colbeck and Warren, 1991). Colbeck has collected many pertinent references into a comprehensive bibliography of articles published up to 1993 (Colbeck, 1993). Since then, an experimental study by Liang and colleagues (Liang et al., 2003, 2005) and its analysis by Dash (2003) have shown that meltwater lubrication may persist down to very low ambient temperatures provided that the frictional energy is sufficient to maintain the local temperature close to the bulk melting point.

Lubrication by the melt liquid may occur in many other materials. For example, recent experimental evidence for dramatic reductions in the frictional resistance of mineral systems at high-slip speeds have been attributed to localized "flash weakening" at highly stressed asperity contacts, which momentarily approach the melting transition even though the average surface temperature remains much lower (Rice, 2006).

A study by Jellinek (1967) was the earliest to evaluate the viscosity of unfrozen layers of water, from the measurements of ice moving over polished surfaces of quartz and steel. At $-4.5^{\circ} \mathrm{C}$ he obtained very high viscosity values: $15-150 \mathrm{P}$ for ice quartz and $70-700 \mathrm{P}$ for ice steel. By contrast, the viscosity of water at $0{ }^{\circ} \mathrm{C}$ is approximately $0.01 \mathrm{P}$. Barer et al. (1980) developed a different method; the motion of an ice plug in a smooth capillary, driven by a pressure difference. The impedance of the plug was modeled by an annular liquid film of thickness $h$ and uniform viscosity $\eta$, combined into 
the parameter $h / \eta$. The authors calculated $\eta$ from the ratio by assuming the theoretical values of $h$ that had been predicted by Fletcher $(1962,1968)$; their values of $\eta$ relative to the viscosity $\eta_{w}$ of supercooled water at the same temperature appeared to increase linearly with temperature difference $\Delta T=T-T_{m}$, reaching $\eta / \eta_{w}=20$ at $-2{ }^{\circ} \mathrm{C}$. Fletcher's approximate formula for thickness $d$ in $\AA$ varies as $d=C-25 \log _{10}(\Delta T)$, with the constant $C$ between 20 and $50 \AA$. However, the thickness of the liquid layer due to surface melting varies as a power law rather than logarithmically; this difference plus the very wide range of $C$ makes the calculated values of $\eta / \eta_{w}$ problematic. The directly measured ratios $h / \eta$ are more trustworthy.

Bluhm et al. (2000) employed atomic force microscopy (AFM) to study ice friction. The ice was deposited from purified water vapor as a thin film on in situ freshly cleaved mica. The temperature range was -24 to $-40{ }^{\circ} \mathrm{C}$, and measurements were carried out under equilibrium vapor pressure. The lateral drag force on ice was found to be greater than that on the bare mica surface; a friction coefficient of 0.6 over the entire temperature region indicated that there was no lubricating water layer. Further tests and calculations indicated that, under the conditions of the experiment, there should have been no effects from pressure melting, surface premelting, or frictional heating. Thus the final conclusion was that the drag measured the "dry friction" of ice.

\section{Faraday, ice, and surface melting}

On 8 September 1842 Michael Faraday recorded his thoughts about snow and ice, and he then began a series of investigations that were to last 20 years. Excerpts from his diary, published many years later (Faraday, 1933) record the beginning of the first scientific investigation into what we now know as surface melting:

"When wet snow is squeezed together, it freezes into a lump (with water between) and does not fall asunder as so much wetted sand or other kind of matter would do."

"In a warm day, if two pieces of ice be laid one on the other and wrapped up in flannel, they will freeze into one piece."

"All this seems to indicate that water at $32^{\circ}$ will not continue as water, if it be between two surfaces of ice touching or very near to each other."

"The ice probably acts as a nucleus, but it appears that the effect of one surface of ice on water is not equal to the joint effect of two."

Although experiments by Faraday and by Tyndall (1856) were proof to them of a liquid film at equilibrium, some contemporaries were unconvinced. As described earlier in this review, James Thomson argued that regelation of ice results from the lowering of the melting temperature due to the temporary increase of pressure at the points of contact, and refreezing when the pressure is relieved.

In an entirely independent line of inquiry, speculations that all solids might begin melting at their surfaces, even under equilibrium conditions, were given by Tammann (1909) and Stranski (1942). The physical motivation was proposed on the basis of a qualitative argument; the reduction of surface energy. The idea was developed years later, and is the basis for the modern theory, as we describe in Sec. II. But apart from theory, Yakov Frenkel was sure that the phenomenon must exist; all one has to do is observe. He wrote (Frenkel, 1946):

"It is well-known that under ordinary circumstances an overheating (of a crystal), similar to the overheating of a liquid, is impossible. This peculiarity is connected with the fact that the melting of a crystal, which is kept at a homogeneous temperature, always begins on its free surface. The role of the latter must, accordingly, consist in lowering the activation energy, which is necessary for the formation of the liquid phase, i.e., of a thin liquid layer, down to zero."

Frenkel's argument seemed plausible, but it remained untested until techniques became adequate to prepare well-ordered crystal surfaces and to detect extremely thin layers of the incipient liquid. We describe some of the principal features of these studies in the following; more detailed descriptions are given in several reviews (Dash, 1988, 1989a; Pluis and denier van der Gon, 1988; Dash et al., 1995; Suzanne and Gay, 1996; Tartaglino et al., 2005).

A highly detailed series of experiments on single crystals of $\mathrm{Pb}$ gave quantitative measures of the depth of surface melting and its temperature range (Frenken and van der Veen, 1985). These studies used the technique of proton backscattering. The backscattered intensity at certain angles is extremely low in good crystals because of blocking by the ordered rows of atoms, so the backscattered intensity is primarily due to surface structural disorder such as in a melted layer. Experiments gave clear evidence of surface disorder on the (110) facet, beginning as one or two atomic layers at $500 \mathrm{~K}$, some $100 \mathrm{~K}$ below the bulk melting point $T_{m}$, and increasing smoothly with temperature and diverging at $T_{m}$. The functional form of the temperature dependence was logarithmic, as $\ln \left(T-T_{m}\right)$, which theory indicates as due to short-range interactions. Similar studies were carried out on $\mathrm{Al}$ and other crystals, with similar results. Calorimetric techniques were used in studies of surface melting of thick films of Ar and Ne (Zhu and Dash, 1986). The films were adsorbed on exfoliated graphite, a substrate that has been useful in many studies of adsorbed films, since it provides high areas of well-ordered basal plane facets. The high area made it possible to detect thermal signals due to melting of the adsorbed films, even as it began with the transition of the topmost layers. The thermal signals indicated that melting began as low as $0.8 T_{m}$ in both Ar and Ne. Analysis indicated that 
the thickness of the melted layer varied as a power law on temperature as $\left(T-T_{m}\right)^{a}$; the exponent $a$ was approximately equal to the value $1 / 3$, indicating nonretarded dispersion forces driving the melting.

One of the first experiments to give a clear indication of surface melting also showed distinct variations with orientation. Stock (1980) observed the thermal radiation from spherical crystals of copper as they were heated. Since the optical constants of metals are discontinuous at the melting point, surface melting was evident in the changes in the light emission. These changes did not occur uniformly over the surface: the areas around the (100) and (111) poles remained free of liquid up to the melting point of the entire crystal. The method is very sensitive; it was estimated that the liquid films on the melted facets were from 2 to 7 atomic layers thick at 1 to 2 degrees below the melting point. A more detailed study of facet dependence was later carried out by Pluis et al. (1987), using proton backscattering. These studies showed strong variations with orientation, and agreed with Stock's finding that (111) planes showed no signs of surface melting even at temperatures close to $T_{m}$. Heyraud and Métois (1987) gave a dramatic demonstration of the stability of (111) planes. They produced microscopic $\mathrm{Pb}$ crystals with shapes completely bounded by (111) planes, and showed that the crystals survived heating to a temperature several degrees above $T_{m}$. Maruyama (1988) also studied orientational variations of surface melting, as it affected the shapes of $\mathrm{Kr}$ and $\mathrm{Xe}$ crystals heated near their melting points. His results indicated that all surface planes melted, in contrast to the anisotropic surface melting of metal surfaces. Studies of surface melting have been carried out on many types of materials, including molecular solids, semiconductors, and organic crystals, and virtually all techniques that have been developed for studies of bulk materials have proven suitable in studies of surface melting. It is now known that surface melting is the way virtually all solids melt. Variants of surface melting-premelting-occur at interfaces with solid substrates, where it is called interfacial melting and at the grain boundaries of polycrystalline materials, denoted as grain-boundary melting.

The physical mechanism of surface melting is described on several levels. Microscopically, it results from the weaker binding of atoms near a free surface, which makes them more susceptible to thermal disorder. As temperature rises the crystalline structure begins to break down in the surface layers, and the increasing number of defects allows atoms to diffuse more readily. As the temperature continues to rise; disorder and mobility increase and propagate inward. When the melting point is neared the melted layer grows into a thick film. It retains some crystalline order within a few molecular distances of the solid interface, but in its upper layers is indistinguishable from the liquid phase. In the transitional zone the liquidlike disorder and fluidity evolve with distance from the solid interface, and in some detailed studies one can see that the changes proceed stepwise, layer by layer. Initially the surface melted film is a quasiliquid, an intermediate state between a solid and a true liquid. As $T$ nears the melting point the thickness increases rapidly, and finally diverges at $T_{m}$. On a macroscopic level, surface melting is a wetting phenomenon, the wetting of a solid surface by its melt liquid. The theory of surface melting from this perspective is given in Sec. II.A.

\section{Background: Relevant phenomena}

\section{Adsorption and wetting}

Adsorption and wetting are closely related to premelting. They all describe fundamental and practical surface phenomena, involving the chemistry and physics of thin films, and the technology of surfactants and interfaces. Adsorption and wetting have a relatively independent scientific history, reaching back to the 19th century, and each has advanced considerably in the modern era. A significant part of their recent development has come from modern studies of adsorbed films. One of the principal motivations is great interest in the physics of lower dimensional matter, for in some cases monolayer films display properties expected of two-dimensional phases and phase transitions. Multilayers are intrinsically more complicated; the rich systematics of multilayer adsorption on attractive substrates has been described by Pandit et al. (1982). A graphic description of the phases has been given by Michael Schick (1990):

"I envisage a container of which one wall is a piston so that the pressure within it can be varied. A known amount of gas is introduced at the temperature $T$ which produces a pressure $P$, or equivalently, a chemical potential $\mu$. We expect that the attractive interaction between the atoms...will cause a film of dense phase, of either solid or liquid, to form on the walls. (To be specific, let the temperature be such that a film of liquid forms.) Now let the pressure, or chemical potential, in the system be increased so that coexistence between bulk gas and liquid is approached. Either the film of fluid becomes macroscopically thick at coexistence or it does not. If the former occurs, then the interface between the wall and the gas is said to be wetted by the liquid.... This happens by the gradual thickening of the film of fluid as coexistence is approached."

If the walls are not wet at coexistence, the system is incompletely wet; the film will only grow to a finite thickness before bulk coexistence is reached, whereupon bulk droplets or crystals appear on top of the finite thickness film. The transition between the two regimes occurs at the wetting temperature. We see a strong correspondence between the two classes of wetting, and complete and incomplete premelting, which are exhibited by ice under some circumstances (see Sec. II.B.1).

\section{Porous media}

William Thomson explained that small samples of a material melt at a lower temperature than the bulk sub- 
stance, due to the combination of surface curvature and surface energy (Thomson, 1871). The shift, now known as the Gibbs-Thomson effect, has been studied with a variety of substances dispersed on inert substrates or confined in porous media. A sampling is given in the references in four groups: weakly interacting molecular species such as rare gas atoms in porous media (Brewer et al., 1978, 1990; Tell and Maris, 1983; Liezhao et al., 1986; Shimoda et al., 1986; Kondo et al., 1987; Brown et al., 1988; Bruschi et al., 1988; Hiroi et al., 1989; Jackson and McKenna, 1990; Rall et al., 1991; Duffy et al., 1995; Beaudoin et al., 1996), ice in porous media (Blachere and Young, 1972; Gay et al., 1992; Maruyama et al., 1992; Ishizaki et al., 1996; Mori et al., 1996), dispersed metal particles (Takagi, 1954; Gladkich et al., 1966; Wronski, 1967; Coombes, 1972; Peppiatt and Sambles, 1975; Buffat and Borel, 1976), and ice in soils (Hoekstra and Delaney, 1974; Konrad and Morgenstern, 1981; Smith and Tice, 1988).

The typical behavior of the first group is that melting begins appreciably below the bulk transition when the pores are filled. Although the first one or two adsorbed layers prefreeze, due to substrate attractive forces stronger than the interactions within the material. The adsorbed layers are disordered for two reasons; the gradient of the substrate attraction, and the curvature of the pore. At moderate distances from the substrate the decreased attraction allows the solid to take on its normal ordered crystal structure. The behavior of ice differs because its cohesive forces are much stronger, and hence adsorption is relatively weak; there may be chemical bonding of the first layer, but no strong adsorption beyond that. Beyond the adsorbed layers, ice and other materials behave similarly.

The solid may be finely polycrystalline immediately after filling and freezing, but it gradually develops into one or a small number of distinct crystals, driven by the tendency to reduce its grain-boundary energy. In the limit, each pore will be filled with one or a small number of crystals inside a cavity lined with a dense film of the same substance, which acts as a thermodynamically distinct, inert wall. Whereas the equilibrium shape of a free crystal of fixed volume, for example, in contact with the bulk liquid phase, is determined by minimizing the total surface free energy $\gamma_{s \ell}(\phi)$ which depends on the orientation $\phi$ of the surface with respect to the underlying lattice, the shape of the confined solid is determined by the pore geometry. The solid's equilibrium orientation minimizes the interfacial energy, which is a convolution of the polar angle dependence of the surface coefficient of the solid-wall boundary $\gamma_{s w}(\phi)$ with the geometry of the pore. The regions with large interfacial coefficient $\gamma_{s w}$ have strongly negative wetting coefficients $\Delta \gamma$, and it is at these regions where premelting begins when the crystal is warmed. Where the core is composed of a number of crystals, and at the junctions between adjacent pores, premelting may occur in the grain boundaries as well as at the wall interface. As the temperature rises melting spreads to other orientations, and the remaining solid tends toward a free growth shape, floating within, and convex toward its melt liquid. In this limit the solid melts abruptly at a temperature $T$ below the bulk transition $T_{m}$. The shift of the melting temperature for an "isotropic" solid with interfacial curvature $\mathcal{K}$ is given by the equation

$$
T_{m}-T=\frac{\kappa_{v} \gamma_{s \ell} T_{m}}{\rho_{s} q_{m}} \mathcal{K}
$$

where, as above, $\gamma_{s \ell}$ is the interfacial free energy between the liquid and solid. The effect of second-order terms is treated by Mori et al. (1996). The term $\kappa_{v}$ arises from the pressure dependence of $T_{m}$; if the system is in equilibrium with the vapor,

$$
\kappa_{v}=1-\frac{(d P / d T)_{s v}}{(d P / d T)_{s \ell}},
$$

where the pressure slopes are the values at the solidvapor and solid-liquid phase boundaries. The ratio is typically quite small; in $\mathrm{H}_{2} \mathrm{O}$, for example, $\kappa_{v}$ differs from unity by about $3 \mathrm{ppm}$. Thus, we see from Eq. (1) that for a solid-liquid interface convex $(\mathcal{K}>0)$ into the melt phase the freezing point is depressed and there is an enhancement for concave interfaces. The case of a sphere of radius $r$ provides the case most commonly known as the Gibbs-Thomson equation (Thomson, 1871):

$$
T_{m}-T=\frac{\gamma_{s \ell} T_{m}}{\rho_{s} q_{m}} \frac{2}{r}
$$

Many experiments have demonstrated that the melting temperatures of materials are depressed in porous media, due to the Gibbs-Thomson effect. Such experiments (noted in Sec. IV.D, which includes a review of a highly detailed analysis of the melting of ice in powders) obtained close quantitative agreement with the measured liquid fraction. A host of studies of freezing soils have been motivated by the serious hazards of frostheave to engineered structures in temperate and cold climates. See Sec. II.D.1 for the theory of frost-heave pressure, and Sec. V.A.1 for a description of its environmental effects.

\section{Premelting and phase change}

It is important to distinguish premelting, with its varieties surface, interfacial, and grain-boundary melting, from other types of premonitory melting, where liquid appears on the wrong side of the bulk phase boundary. The sharpness of the bulk melting transition can be broadened and shifted by impurities, dislocations, polycrystallinity, and other forms of disorder. In contrast, premelting is an equilibrium phenomenon of wellordered crystal surfaces; indeed, it was only when surfaces of sufficient purity and perfection were available that the effect could be definitely observed in isolation.

Surface melting is not a distinct phase change, but is instead a surface aspect of the bulk transition; a "surface phase-transition." Hence, although melting is a firstorder phase change, it is initiated with the gradual de- 
velopment of surface disorder and mobility. As the temperature is raised toward the bulk transition $T_{m}$ the disorder and mobility increase and progress deeper into the solid. The disordered region is a quasiliquid: when it is microscopically thin it retains the structure of the underlying solid, but when it thickens the outer layers become identical to the bulk liquid. In the intermediate zone the liquidlike disorder and fluidity evolve with distance; in some cases the evolution is stepwise, in monolayer increments (Chernov and Mikheev, 1989; Phillips, 1989). It is even possible to see that the transition advances as a kind of two-dimensional melting within each layer. And even within this progression, there is a lowerdimensional premelting-edge melting-as each layer undergoes its transition (Pengra and Dash, 1992). When the temperature reaches $T_{m}$ the transition zone migrates inward, leaving bulk liquid in its wake. Thus the fully developed quasiliquid obtains the width or diffuseness greater than that of the solid-liquid interface (Burton et al., 1950; Jackson, 1958; Cahn, 1960). Crystal-liquid interfaces have been studied by molecular dynamics (Broughton et al., 1981; Phillips, 1989) and a variety of theoretical techniques (Haymet and Oxtoby, 1974; Curtin and Ashcroft, 1985; Karim and Haymet, 1987; Shih et al., 1987). A molecular-dynamics simulation of a Lennard-Jones fcc crystal found that the crystalline order falls to 1/e in approximately four atomic layers (Karim and Haymet, 1987), and an experimental measurement of the latent heat of $\mathrm{Ar}$ and $\mathrm{Ne}$ films found that the entropy change has a decay length of five atomic layers (Zhu and Dash, 1988). Measurements of ice growth (Maruyama et al., 1997) have shown that the width of the water-ice interface is anisotropic; in the direction of the $c$ axis it is molecularly sharp, whereas more rapid growth normal to the $c$ axis indicates a rougher and greater interface width (Cahn et al., 1964).

The diffuseness of the solid-liquid interface limits the mean-field theory range of surface melting, which approximates the surface melt as a thin slab of the bulk (see Sec. II.A). Although the approximation is strictly valid for melt layer thicknesses greater than the interface width, the theory in some cases can provide a moderately successful description of the quasiliquid down to thicknesses of one or two layers (Frenken and van der Veen, 1985; Zhu and Dash, 1986).

Further connections between surface melting and the bulk transition are discussed in a recent review (Dash, 2002), the role of surface melting on crystal growth is discussed in Sec. IV.E, and the closely related issue of surface roughening is discussed presently.

\section{Crystal shapes and roughening}

The foundation of our understanding of the equilibrium forms of ice crystals arose from attempts to predict their growth shapes (Wulff, 1901). As mentioned in Sec. I.D.2, the shape that minimizes the total interfacial free energy at fixed volume is the equilibrium crystal shape. An ideal, dislocation-free crystal is fully faceted at absolute zero, and becomes more rounded, or locally rough, as its temperature increases [reviews can be found in Weeks and Gilmer (1979), Lipson and Polturak (1987), and Balibar et al. (1993, 2005)]. Using experiment and exact solutions of microscopic models allows one to construct the surface free energy of a crystal from the orientation-dependent bond energies. Hence, whether the crystal is in contact with the vapor or the liquid phases, the interfacial free energy $\gamma(\phi)$ is considered as the sum of the energies of all bonds broken per unit area in the creation of the surface of an orientation $\phi$ relative to the underlying crystalline lattice. Were one given the surface free energy of the crystal as a function of all orientations present, the Wulff construction would provide the equilibrium crystal shape (Wulff, 1901; Herring, 1951a). On the other hand, were one to measure the shape of a crystal in equilibrium, Wulff's construction produces the surface free energy for all orientations present on that shape (e.g., Heyraud and Métois, 1986). Ice in contact with the melt phase and negative crystals of ice beautifully exhibit these points (Koo et al., 1991; Furukawa and Kahota, 1993).

How then does the equilibrium crystal shape evolve from fully facetted to rounded and rough as the temperature rises? The surface is described by the free energy of the distribution of configurations, viz., faces, ledges, corners, edges, and point defects such as admolecules and vacancies. A given state of the surface weighs the cost of the formation enthalpies of the sites against the reduction of the total free energy derived from increasing the configurational entropy of the surface: this is the driving force for the thermodynamic roughening transition. The creation of surface sites is an activated process and the coherence length $\xi(T)$ is a measure of the distance, relative to the mean orientation of the surface, over which fluctuations at two points are correlated (e.g., Lipson and Polturak, 1987). As the temperature $T$ increases, the step-free energy $\sigma_{s}(T)$ decreases. For an infinite two-dimensional surface $\sigma_{s}(T) \rightarrow 0$ at the roughening temperature $T_{r}$ and hence the coherence length $\xi(T)=\gamma(\phi) / \sigma_{s}(T)$ diverges; thermal fluctuations liberate the surface from the ordering influence of the underlying crystalline lattice, they are correlated on all length scales and therefore the surface roughens. The process is reigned in by the finite size of the crystal; as the temperature rises and $\xi(T)$ approaches the facet size, a given facet will roughen at $T=T_{f}<T_{r}$ and hence if $T>T_{f}$ for all facets present, an equilibrium shape will be completely rough and rounded.

Roughening transitions have been observed in ice against two relevant bulk phases, water and vapor. The roughening transition of the prism facet of ice $I_{h}$ was found under vapor conditions by Elbaum (1991a), and Maruyama et al. (1997) performed a series of experiments over a large range of pressure and temperature for ice in contact with water. Indeed, both the roughening transition of the prism facet of ice $I_{h}$ and changes of the equilibrium crystal shape were found by Maruyama et al. (1997) through careful control along the solidliquid coexistence line from near the triple point to 
$-21{ }^{\circ} \mathrm{C}$ at $200 \mathrm{MPa}$. They discovered that $T_{f}=-16^{\circ} \mathrm{C}$ at $165 \mathrm{MPa}$ for prism facets. Because their approach provides accurate control of the growth drive very near equilibrium it offers a reliable test of theoretical predictions of crystal shapes growing and melting near equilibrium (Sec. IV.E). Some of the same ideas involved in surface roughening are involved in surface melting, the thermodynamics of which we describe presently.

\section{THEORY}

\section{A. Thermodynamics of premelting}

The physical motivation for surface melting is the reduction of interfacial energy. The general mechanism was proposed long ago, on the basis of qualitative arguments (Tammann, 1909; Stranski, 1942; Frenkel, 1946), but the principles of the modern theory were explicitly stated only many years later (Kristensen and Cotterill, 1977; Kuroda and Lacmann, 1982; Broughton and Gilmer, 1983a, 1983b, 1983c, 1984a, 1984b; Lipowsky and Speth, 1983; Furukawa et al., 1987; Nenow and Trayanov, 1989). Premelting is the general term for the phenomenon which can occur at three different classes of interface: surface melting between a solid and its vapor or gaseous atmosphere, interfacial melting in contact with foreign solid or liquid, and grain-boundary melting between crystals of the same material. Several extensive reviews have been presented from a variety of perspectives (Dietrich, 1988; Sullivan and Telo da Gama, 1988; van der Veen et al., 1988; Lowen 1994; Dash et al., 1995; Tartaglino et al., 2005); presently, we review the theory from the standpoint of the thermodynamics of wetting (Schick, 1990).

If the interface is wetted by the liquid phase (meaning that a layer of the liquid phase intervenes between the solid and vapor or foreign substrate), it implies that the free energy of the wetted boundary is lower than it would be without the liquid. We then infer that a wetting layer will persist over some finite temperature range below the normal melting point. For if the state of the system slightly below the bulk melting temperature were to be initially dry, the system could lower its free energy by converting a layer of the solid to liquid. The cost of this conversion involves the free energy change due to melting, but if the layer is thin enough and the temperature sufficiently close to the bulk melting point the cost will not be prohibitive. This competition between surface and conversion terms establishes the actual thickness of the liquid; it is the value at which the free energy of the system is at a global minimum. It is important to note that in the theory which follows, it is assumed that the layer is sufficiently thick that its chemical potential is approximated as that of the bulk liquid. As discussed in Sec. I.D.3, the melt layer retains a certain amount of solidlike order when it is very thin, and gradually loses its remnants as it thickens. The change typically extends over several layers; in the case of $\mathrm{Ar}$ and $\mathrm{Ne}$ it is exponential, with a decay length of five or six atomic layers. The change is more rapid in ice; parallel to the $c$ axis it is approximately three layers, and somewhat longer in normal directions (Cahn, 1960; Cahn et al., 1964; Furukawa and Nada, 1997; Nada and Furukawa, 1997). Until the vestiges of solidlike order are lost the melt is a quasiliquid. In the following treatment the distinction between the quasiliquid and a true liquid is suppressed. However, as noted in the discussion of laboratory studies, the approximation is quite successful in extending the theory down to very thin melts.

Consider a solid in equilibrium with a vapor at temperature $T$ and pressure $P$. If the interface is wetted by a macroscopic quasiliquid layer $(q \ell \ell)$, the free energy of the layer is composed of bulk and surface terms. The free energy per unit area can be written as

$$
G_{q \ell \ell}(T, P, d)=\left[\rho_{\ell} \mu_{\ell}(T, P)\right] d+F_{\text {total }}(d),
$$

where $d$ is the film thickness, $\rho_{\ell}$ and $\mu_{\ell}$ are the molecular density and chemical potential of the bulk liquid $(\ell)$, and $F_{\text {total }}(d)$ is the total excess surface free energy per unit area [the effective interfacial energy in mean-field theory (Schick, 1990)]. Its value varies from $\gamma_{s v}$, the coefficient of the unwetted solid-vapor interface, at $d=0$, to the sum of the solid-liquid and liquid-vapor coefficients $\gamma_{s \ell}+\gamma_{\ell_{v}}$ at $d=\infty$. Its functional dependence on $d$ reflects the type of basic interactions in the material. A phenomenological description that brings out the variation more explicitly can be written as

$$
F_{\text {total }}(d)=\Delta \gamma f(d)+\gamma_{s v},
$$

where the wetting parameter $\Delta \gamma$ is the difference between the coefficients of the dry and wetted interface:

$$
\Delta \gamma \equiv \gamma_{\ell v}+\gamma_{s \ell}-\gamma_{s v} .
$$

Premelting requires that $\Delta \gamma<0$ (see the Appendix of Wettlaufer and Worster, 1995). The film-thicknessdependent contribution $f(d)$ ranges from 0 to 1 as $d$ increases from 0 to $\infty$.

In thermodynamic equilibrium, the chemical potential of the melted layer is equal to that of the solid. Substituting Eqs. (5) and (6) into Eq. (4) and differentiating with respect to $d$,

$$
\mu_{q \ell \ell}=\mu_{\ell}(T, P)+\left(\frac{\Delta \gamma}{\rho_{\ell}}\right) \frac{\partial f}{\partial d}=\mu_{s}(T, P) .
$$

The interfacial term in Eq. (7) introduces a difference between the chemical potentials of the solid and bulk liquid, which displaces the location of the thermodynamic coordinates from the normal phase boundary. The displacement can be calculated by a Taylor series expansion of the chemical potential in powers of temperature and pressure about the normal equilibrium coordinates at the melting point $T_{m}, P_{m}$. To first order,

$$
\Delta \mu(T, P)=\left[\frac{\partial \Delta \mu}{\partial T}\right]_{T_{m}}\left(T-T_{m}\right)+\left[\frac{\partial \Delta \mu}{\partial P}\right]_{P_{m}}\left(P-P_{m}\right)
$$




$$
=\frac{q_{m}}{T_{m}}\left(T-T_{m}\right)-\left(\frac{1}{\rho_{\ell}}-\frac{1}{\rho_{s}}\right)\left(P-P_{m}\right),
$$

where $q_{m}$ is the latent heat of melting per molecule and $\Delta \mu(T, P) \equiv \mu_{s}(T, P)-\mu_{\ell}(T, P)$. The pressure shift can be expressed in terms of the temperature shift and the slopes of the sublimation and melting lines, through the Clausius-Clapeyron relation. Equation (8) can then be transformed to

$$
\Delta \mu=\kappa_{v} q_{m}\left(T-T_{m}\right) / T_{m},
$$

where $\kappa_{v}$ is the same as in Eq. (2). We noted there that for ice $\kappa_{v}$ is essentially unity.

Equation (10), together with the specific form of $f(d)$, leads to the theoretical expression for thickness. In typical molecular substances with unretarded dispersion, or van der Waals forces, the long-range potentials fall off quadratically with distance, and thus we can write

$$
f(d)=1-\frac{\sigma^{2}}{d^{2}},
$$

where $\sigma$ is a constant on the order of a molecular diameter. In this case, the temperature dependence of thickness in the range $d \gg \sigma$ leads to

$$
d=\left(-\frac{2 \sigma^{2} \Delta \gamma}{\rho_{\ell} q_{m}}\right)^{1 / 3} t^{-1 / 3},
$$

where $t=\left(T_{m}-T\right) / T_{m}$ is the reduced temperature. In the case of short-range forces, $\partial f / \partial d \propto \exp (-c d)$ where $c$ is a constant. The thickness then varies logarithmically with temperature as $d \propto|\ln (t)|$. Other interactions, such as those due to ions, can produce other similarly specific temperature dependences. We discuss these interactions in more detail in the following section.

\section{B. Lifshitz theory of ice premelting}

\section{Pure ice at the vapor surface; incomplete surface melting}

In this section we focus on a theory of premelting for those systems whose interactions are dominated by dispersion forces. The so-called Lifshitz theory (Dzyaloshinskii et al., 1961) has had remarkable success in predicting the nature of the surface melting of ice, providing the distinction between complete and incomplete surface melting. When melt layers at certain solid surfaces diverge smoothly at the bulk transition the melting is complete but where retarded potential effects intervene and attenuate the intermolecular wetting forces the film growth may be blocked and thereby be finite at the bulk transition. This latter circumstance, in which the behavior is discontinuous, is referred to as incomplete melting.

The entire range of experimental studies in ice is detailed in Sec. IV, but in order to demonstrate the basic effects of dispersion forces-frequency-dependent van der Waals interactions - on the surface of ice, we point out a key experimental finding, leading to the conclusion that ice does not surface melt completely at all orientations. In two experiments (Ketcham and Hobbs, 1969;
Knight, 1971) direct visual observation was made of standing droplets of water, with small but finite contact angle, at temperatures just below the triple point. A study using optical reflectometry and interference microscopy (Elbaum et al., 1993) examined the surfaces of prism and basal facets, in pure water vapor and in air. Basal facets in pure vapor showed surface melting beginning at or slightly below $-2{ }^{\circ} \mathrm{C}$ whereas above $-2{ }^{\circ} \mathrm{C}$ thickness increased with $T$ up to a few hundredths of a degree below bulk melting, when droplets, also with small contact angle, suddenly appeared on the surface. The droplets were stable and reproducible on temperature cycling. The experiment demonstrated the incomplete wetting of the quasiliquid film by the bulk liquid. When air was introduced into the chamber the surface melted completely. We use this as the backdrop to explain the theory of wetting under frequency-dependent van der Waals interactions.

When the polarizability of the substrate is greater than that of the film, wetting occurs. Thus, generally speaking, when dispersion forces dominate, the wetting of any ice surface by water at temperatures below $T_{m}$ will be facilitated when the polarizability of the water lies between that of the ice and the other material, be it a gaseous phase, a chemically inert solid, or ice. The net wetting forces depend on the entire range of frequencies that underlie the polarizability of the layered system. Indeed, a particular novelty of the system, first pointed out by Elbaum and Schick (1991) in their study of the surface melting of ice, is that the appropriately transformed polarizability of ice is greater than that of water at frequencies higher than the ultraviolet (approximately $2 \times 10^{16} \mathrm{rad} \mathrm{s}^{-1}$ ), whereas it is smaller at lower frequencies. Hence, so long as the surface melted layer of water is thin, the polarizabilities at all frequencies contribute additively to the wetting forces, whereas upon thickening retardation - the attenuation of the interaction due to the finite speed of light-reduces the high-frequency contributions and favors those in which the polarizability of water dominates over that of the ice. Then cohesion, the self-attraction of the water, wins out, and the adhesion to the ice stops at some limiting thickness; wetting is incomplete. Thus the frequency dependence of the wetting forces explains the coexistence of droplets of water on surface melted films. Many solids have dielectric properties that lead to complete interfacial premelting whereas the pure vapor phase of ice does not. We now detail the underlying theory.

As described above, complete interfacial melting is determined by the competition between bulk and surface-free energies. It requires that the total excess surface-free energy per unit area $F_{\text {total }}(d)$ be a positive monotonically decreasing function of the film thickness with a global minimum at infinite film thickness. In general, it is conceptually useful to consider the thicknessdependent contributions to $F_{\text {total }}(d)$ to arise from ostensibly short- and long-ranged intermolecular interactions: namely, as $F_{\text {total }}(d)=\gamma_{s \ell}+\gamma_{\ell v}+F_{\text {short }}(d)+F_{\text {long }}(d)$, where the interfacial coefficients make implicit reference to the crystallographic orientation present at the interface. 
Hence, by definition, at large enough distances, the long-range interactions always dominate over shorterrange interactions. If interactions underlying $F_{\text {long }}(d)$ are attractive and hence represented by a negative monotonically increasing function of $d$, then $F_{\text {total }}(d)$ can never have a global minimum at $d=\infty$. However, if a short-range interaction $F_{\text {short }}(d)$ favors melting, it is possible that it may prevail over $F_{\text {long }}(d)$ out to large film thicknesses; $\left|F_{\text {short }}\left(d^{\prime}\right)\right| \geqslant\left|F_{\text {long }}\left(d^{\prime}\right)\right|$ for $0 \leqslant d \leqslant d^{\prime}$. Thus the magnitude of the global minimum in $F_{\text {total }}(d)$ may be quite small but is, nonetheless, realized at a large, but finite, value of $d \equiv d_{\min }$. In other words, $F_{\text {total }}\left(d_{\min }\right)$ $\equiv \min \left[F_{\text {total }}(d)\right] \equiv \Gamma$. If this were the case, most physical observations of the system would quite possibly be, depending on the nature of the probe used, virtually indistinguishable from those expected for complete melting. In this section we review the understanding of the longranged contributions to the total wetting force $F_{\text {long }}(d)$, and we bring in the role of the short-ranged intermolecular interactions $F_{\text {short }}(d)$ in the context of grainboundary melting and impurity effects.

When only nonretarded van der Waals forces contribute to the total excess surface free energy per unit area, they commonly take the form $F_{\text {long }}(d) \equiv F_{\mathrm{vdW}}^{\mathrm{NR}}(d)$ $=-A_{H} / 12 \pi d^{2}$, where $A_{H}$ is the Hamaker constant (e.g., Israelachvili, 1992). In general, for dissimilar materials, the van der Waals contribution can be both attractive and repulsive and one can observe oscillations in the force versus distance/film thickness curve leading to the possibility of the system being trapped in a local rather than global minimum (Elbaum and Schick, 1991; BarZiv and Safran, 1993; Wilen et al., 1995; Fenzl, 2003). Such is the case of the surface and interfacial melting of ice (Elbaum and Schick, 1991; Wilen et al., 1995) which demands a more complete calculation of dispersion or van der Waals forces.

The frequency-dependent dispersion force contribution to the free energy of a surface consisting of a pure liquid water layer of thickness $d$ between bulk ice and the vapor phase is treated within the context of the socalled Lifshitz theory. The approach is a continuum quantum electrodynamic theory due to Dzyaloshinskii, Lifshitz, and Pitaevskii (1961) (DLP). The result of this theory is an integral expression for $F_{\mathrm{vdW}}(d)$, in terms of the frequency-dependent dielectric polarizabilities of ice $(s)$ and water $(\ell)$. As described above, were surface melting under dispersion forces alone to be considered, then $F_{\text {total }}(d)=\gamma_{s \ell}+\gamma_{\ell v}+F_{\text {long }}(d)$ where here $F_{\text {long }}(d)$ $\equiv F_{\mathrm{vdW}}(d)$. Complete surface melting would be indicated by a global minimum of $F_{\mathrm{vdW}}(d)$ at $d \rightarrow \infty$ so that $F_{\text {total }}(d)=\gamma_{s \ell}+\gamma_{\ell v}$ at bulk coexistence. Incomplete melting under dispersion forces is indicated by a minimum at finite $d$, with $F_{\mathrm{vdW}}(d)$ negative there.

Although it has appeared in a variety of forms in the literature (Dzyaloshinskii et al., 1961; Elbaum and Schick, 1991; Bar-Ziv and Safran, 1993; Wilen et al., 1995; Benatov and Wettlaufer, 2004), for the sake of selfcontainment we briefly present the DLP expression for
$F_{\mathrm{vdW}}(d)$. The frequency-dependent dielectric response of the layered system is described by

$$
\begin{aligned}
F_{\mathrm{vdW}}(d)= & \frac{k T}{8 \pi d^{2}} \sum_{n=0}^{\infty} \int_{r_{n}}^{\infty} d x x\left\{\ln \left[1-\left(\frac{x-x_{s}}{x+x_{s}}\right)^{2} e^{-x}\right]\right. \\
& \left.+\ln \left[1-\left(\frac{\epsilon_{s} x-\epsilon_{\ell} x_{s}}{\epsilon_{s} x+\epsilon_{\ell} x_{s}}\right)^{2} e^{-x}\right]\right\},
\end{aligned}
$$

where

$$
x_{s}=\left[x^{2}-r_{n}^{2}\left(1-\frac{\epsilon_{s}}{\epsilon_{\ell}}\right)\right]^{1 / 2},
$$

and the material $(s, \ell)$ dielectric functions, corresponding to ice $\left(\epsilon_{s}\right)$ and water $\left(\epsilon_{\ell}\right)$, are evaluated at the sequence of imaginary frequencies $i \xi_{n}=i(2 \pi k T / \hbar) n$. The prime on the sum indicates that the $n=0$ term is weighted by $1 / 2$. The lower limit of integration is $r_{n}$ $=2 d\left(\epsilon_{\ell}\right)^{1 / 2} \xi_{n} / c$, and $k, \hbar$, and $c$ have their usual meaning. The dielectric function required in the integral $\epsilon(i \xi)$ is the analytic continuation of the material dielectric function $\epsilon(\omega)$ to imaginary frequencies. Lacking complete spectra for ice and water, as can be obtained in highmelting temperature materials (e.g., French, 2000) we must generate the function by fitting the dielectric response of the material to a damped-oscillator model of the form

$$
\epsilon(\omega)=1+\sum_{j} \frac{f_{j}}{e_{j}^{2}-i \hbar \omega g_{j}-(\hbar \omega)^{2}},
$$

where $e_{j}, f_{j}$, and $g_{j}$ are fitting parameters (Parsegian, 1975). Each term in the sum corresponds to an absorption band of frequency, width, and oscillator strength, $e_{i}$, $g_{j}$, and $f_{j}$, respectively. Substitution of $i \xi$ for $\omega$ gives $\epsilon(i \xi)$, a well-behaved, monotonically decreasing, real function of $\xi$. We note that Eq. (13) assumes implicitly that $\epsilon_{s}$ is isotropic. Although the effect of the crystal orientation has been studied theoretically (Dal Corso and Tosatti, 1993), the relevant polarization data are not available over the full frequency range. However, judging from known values at optical frequencies (Hobbs, 1974), it is estimated to be small, and hence one generally treats ice crystals as continuum dielectrics.

Retardation in this context can be clearly described by considering a general ice/water $/ X$ interface in which $X$ denotes a "substrate" which may be pure water vapor, for example (Parsegian, 1975; Elbaum and Schick, 1991; Bar-Ziv and Safran, 1993; Wilen et al., 1995; Benatov and Wettlaufer, 2004). When $\epsilon_{\ell} \approx \epsilon_{s} \approx \epsilon_{X} \approx 1$, one can approximate Eq. (13) as

$$
F_{\mathrm{vdW}}(d) \approx-\frac{k T}{8 \pi d^{2}} \sum_{n=0}^{\infty},\left(\frac{\epsilon_{s}-\epsilon_{\ell}}{\epsilon_{s}+\epsilon_{\ell}}\right)\left(\frac{\epsilon_{X}-\epsilon_{\ell}}{\epsilon_{X}+\epsilon_{\ell}}\right)\left(1+r_{n}\right) e^{-r_{n}} .
$$

The term $e^{-r_{n}}$, due to retardation, acts as a highfrequency cutoff to the sum which is inverselydependent on $d$. 


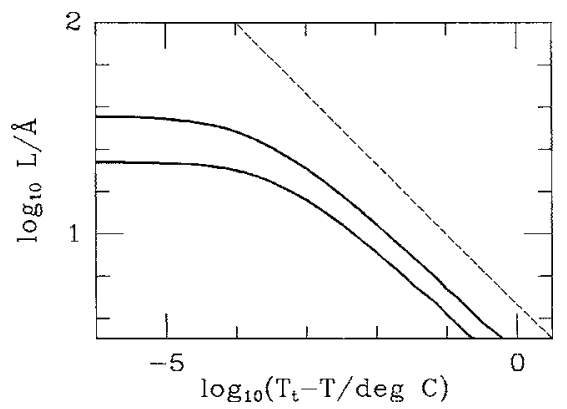

FIG. 1. Calculations of the film thickness vs $T-T_{t}$, with $T_{t}$ the triple point temperature referred to in this paper as $T_{m}$. The dashed line is drawn to show the $\left(T-T_{t}\right)^{-1 / 3}$ power law expected when retardation is unimportant. The upper curve corresponds to use of the data of Daniels (1971), and the lower to data of Seki et al. (1981). From Elbaum and Schick, 1991.

When the substrate is pure water vapor $\epsilon_{X}$ may be taken equal to 1 . In this case, it is clear that were $\epsilon_{s}$ $-\epsilon_{\ell}<0$ at all frequencies, $F_{\mathrm{vdW}}(d)$ would be a monotonically increasing function of $d$ and the film would not grow. Conversely, if $\epsilon_{s}-\epsilon_{\ell}>0$ at all frequencies, then $F_{\mathrm{vdW}}(d)$ would be a monotonically decreasing function and the film thickness would diverge as the melting temperature is approached. Because, as described above, $\epsilon_{s}-\epsilon_{\ell}$ changes sign at frequency $\xi_{c}$, the resulting melting behavior is intermediate between these cases. In particular, for sufficiently large $d$, where the sum is dominated by low-frequency terms, surface melting is inhibited, as is shown in Fig. 1 from Elbaum and Schick (1991).

\section{Pure ice at chemically inert walls: Interfacial melting}

Of broad relevance in environmental and geophysical phenomena is the case when the vapor phase is replaced by a substrate. This is a basic aspect of the physics of relevance in settings ranging from heterogeneous nucleation to ground freezing, and hence allows one to isolate the role of long-ranged wetting forces when substrate heterogeneity and impurity effects are absent. Thus the formalism provided by Eqs. (13) and (16) is appropriate.

For substrates with arbitrary dielectric properties, the results can be complicated due to the fact that the function $\epsilon_{X}$ depends on frequency $\xi$, and $\epsilon_{\ell}(i \xi)-\epsilon_{X}(i \xi)$ may change sign. A systematic study has been carried out for cases in which materials in contact with ice are conductors (gold, copper, silver, tungsten, silicon), dielectric crystals ( $\mathrm{MgO}$, sapphire, fused quartz), and polymers (polyvinylchloride, Teflon, polystyrene, among others) (Wilen et al., 1995). For materials studied previously (Wilen et al., 1995), we observed that $\epsilon_{X}>\epsilon_{s, \ell}$ in the frequency range $\xi<\xi_{c}$. Hence we found that for $d$ large enough $(\approx 30 \AA)$ to allow retardation to come into play, $F_{\mathrm{vdW}}(d)$ will be a positive monotonically decreasing function of $d$, implying fulfillment of the necessary condition for complete interfacial melting. Whether or not complete melting occurs in a given experimental setting for a given substrate will depend on the interactions at short range, $F_{\text {short }}(d)$. When taken alone, the van der
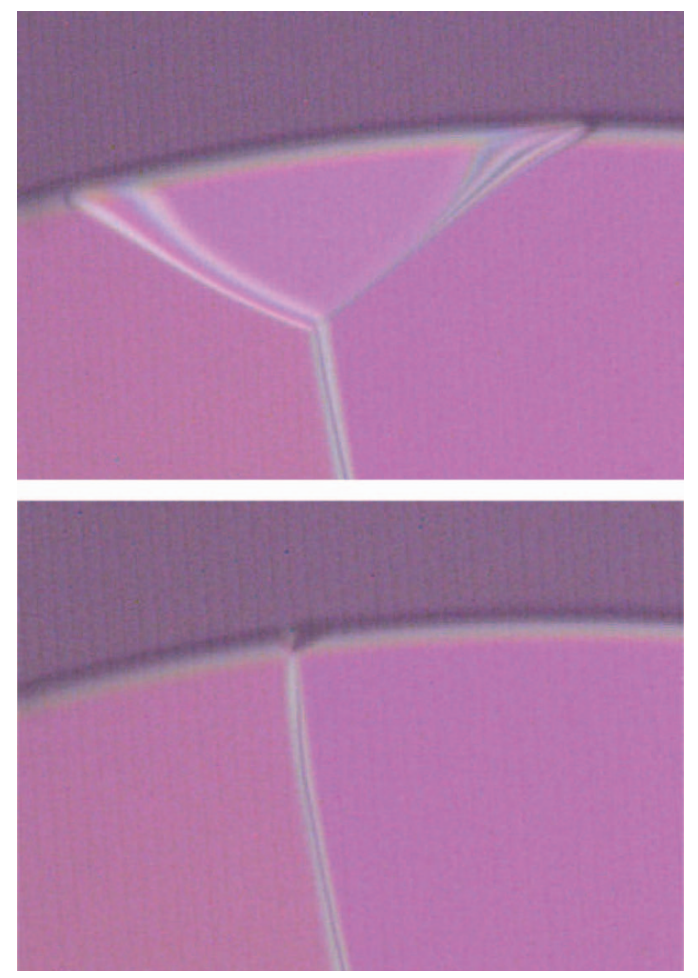

FIG. 2. (Color) Top image: A photograph of a tricrystal of ice in contact, at $T_{m}$, with the bulk liquid (the dark band above the crystal). Under polarized light the birefringence of ice delineates different crystallographic orientations by color. Bottom image: The bicrystal coarsened from the tricrystal after approximately $7 \mathrm{~h}$. The scene is $3 \mathrm{~mm}$ in width. Ice can be held near its melting temperature for a host of experimental studies. From Thomson et al., 2005.

Waals interaction predicts incomplete melting, via a global minimum at finite $d$, for some substrates, and complete melting for others. Where complete melting is indicated, it is found that the film thicknesses are small at temperatures below $-0.1^{\circ} \mathrm{C}$. Indeed, specific types of electrical interactions modeled, for example, using Poisson-Boltzmann theory, can be shown to be much stronger than the van der Waals interaction. Such interactions are required in order to explain grain-boundary melting.

\section{Grain-boundary melting in pure ice}

Grain-boundary melting figures prominently in the sintering, coarsening, transport behavior, and many other bulk properties in ostensibly all materials (Smith, 1948). Indeed, because most materials exist in a polycrystalline state, the implications are vast, ranging from the mechanical and thermal properties of glaciers (Nye and Frank, 1973; Nye, 1991a; Mader, 1992a) to the reduction in the critical current density in hightemperature superconductors (Thompson et al., 2004). Despite the recognition of this fact, the great difficulty of directly accessing a grain-boundary in thermodynamic equilibrium has seriously limited experimental tests. However, the birefringence of ice allows the distinction 
of grains of differing orientations and with optical methods it is hoped that a clear signal of grain-boundary melting in thermodynamic equilibrium will be realized (Thomson et al., 2005). Figure 2 provides such an example.

At temperatures near but below $T_{m}$, polycrystalline matter is threaded by the liquid phase. This liquid is present solely due to the impurity and curvature depressions of the freezing point [see, e.g., Mader (1992a); Nye (1991a), for ice; and Smith (1948), for metals]. The unfrozen water is observed in $10-100 \mu \mathrm{m}$ veins where three grains abut and at nodes separating four grains (see Fig. 11). Nye and Frank (1973) predicted the geometry of the network for isotropic interfacial energies: In exact analogy with the classical Young-Dupré equation for the force-balance used to determine the contact angle of a partially wetting fluid on a substrate or the meniscus in capillary rise (e.g., de Gennes, 1985; Schick 1990), they considered the force-balance at the trijunction where three grains come together. This provides an expression for the dihedral angle $2 \theta_{0}$ into which water intrudes, in terms of the simple ratio of the grainboundary $\gamma_{s s}$ and solid-liquid $\gamma_{s \ell}$ interfacial energies:

$$
2 \cos \theta_{0}=\frac{\gamma_{s s}}{\gamma_{s \ell}}
$$

thereby determining the shape and cross section of a vein. Grain-boundary melting links the concept of interfacial premelting and the vein-node network, for during complete grain-boundary melting the dihedral angle vanishes and the single grain boundary is replaced by two interfaces; $\gamma_{s s} \rightarrow 2 \gamma_{s} \ell$. This process is equivalent to the growth of a liquid film at the grain boundary. In Sec. V.A.5 we describe the environmental implications of this network.

Experimental studies of grain-boundary melting are centered on dihedral angle measurements, principally due to the difficulty of direct access to the equilibrium interface of a bicrystal in a manner free of compromises associated with the proximity of surfaces associated with apparatus. Although computer simulations and theory support the notion of disorder at a grain boundary (Kikuchi and Cahn, 1980; Broughton and Gilmer, 1986; Schick and Shih, 1987; Lobkovsky and Warren, 2002), experimentally it is often stimulated using impurities and then quenching a system before probing the boundary (e.g., French, 2000; Luo and Chiang, 2000). Experiments in aluminum using electron microscopy have shown that as the temperature rises, the boundary structure remains epitaxial until $T=T_{m}$ when the grain boundary has the signature of bulk liquid (Hsieh and Balluffi, 1989). Dihedral angle experiments on bismuth bicrystals placed in contact with the melt were performed for various crystallographic mismatches by Glicksman and colleagues [see Vold and Glicksman (1972), and references therein]. They interpreted their data to indicate a discontinuous grain-boundary melting transition as a function of grain mismatch. The dual importance of grain-boundary melting in materials in gen- eral and its propitious geophysical importance make the problem alluring.

In the context of the DLP approach, $F_{\text {total }}(d) \equiv \gamma_{s s}(d)$ $=2 \gamma_{s \ell}+F_{\mathrm{vdW}}(d)$, which allows one to estimate both the dihedral angle and temperature at which the film thickness would saturate; each as a function of the thickness $d_{\text {min }}$. The angle $\theta_{0}$ is given by $\cos \theta_{0}=1+\Gamma / 2 \gamma_{s \ell}$, and hence, in the pure case, we estimate that the temperature at which the film thickness saturates is given by $T$ $-T_{m} \approx\left(T_{m} / \rho_{s} q_{m}\right) \Gamma / d_{\min }$, where $\rho_{s}$ and $q_{m}$ are the density and heat of fusion of ice. For the grain boundary, and indeed, any system of identical substrates (e.g., $A / B / A$ ) separated by distance $d$ the Hamaker constant is positive, producing an attraction and consequently grainboundary melting under dispersion forces must be incomplete (Lipowsky, 1986; Schick and Shih, 1987). In a $Z(N)$ model calculation of a twist mismatch grain boundary (Schick and Shih, 1987) it was found that at coexistence the boundary may be wetted not by a liquid, but by a solid of intermediate orientation. The implication is that this is a less costly configuration than wetting by a liquid. Schick and Shih (1987) pointed out that their result is consistent with some molecular-dynamics simulations, and that for van der Waals materials a calculation of the Hamaker constant using dispersion force theory indicates incomplete wetting. The importance is that although a given calculation may indicate that the dry grain boundary should decompose at coexistence, whether or not the new configuration is liquid or a solid of intermediate orientation may depend on the nature of the relaxation, and hence also on the details of the model.

As is shown in Sec. II.B.3, when the film contains an electrolyte, the solid grains may be held at bay by repulsive screened Coulomb interactions against the attractive interactions of dispersion forces. The problem does distinguish itself from many other systems for, as we shall see, in the general case there is no strict separation of the thickness dependence of $\gamma_{s s}(d)$ because $F_{\text {short }}(d)$, which originates in the Coulomb interaction, can be quite long range and thereby effectively compete with $F_{\mathrm{vdW}}(d)$.

\section{Effects of nonideality}

The sensitivity of premelting to imperfections, impurities, and other deviations from pure and perfectly ordered surfaces motivates special attention. Essentially all varieties of imperfection promote premelting by increasing the magnitude of the wetting coefficient or by changing the chemical potentials of the solid or its melt liquid. Disorder has a particularly important influence in the surface melting of ice, owing to its extremely narrow temperature range in pure and ordered crystals. Their sensitivity to perturbations is shown by the qualitative change-from incomplete to complete surface melting when the atmosphere was changed from pure water vapor to humid air (see Sec. II.B.1). Although the exact mechanism for the change, whether pressure shift or 
chemical impurity, is not known, the extreme sensitivity of ice premelting is beyond question. Its great susceptibility to disturbances explains the wide discrepancies among measurements of onset temperatures and melt thickness (see Sec. IV). In the following subsections we examine the effects of specific types of disorder.

\section{Nonplanar substrates}

The effects of deviations from smooth planar substrates depend on the scale of the deviation. The most fine-grained phenomena are at the atomic scale, such as chemisorption and epitaxy, and are treated under the rubric of adsorption (see Sec. I.D.1). Their range is typically limited to the first few layers next to the substrate, which for the present we consider part of the substrate itself.

The next coarser scale involves irregular variations in profile that are described as "roughness" which affects a wide class of wetting phenomena (Beaglehole and Wilson, 1994; Netz and Andelman, 1997). Roughness increases the surface area $A$ of the solid-substrate interface, thereby raising the solid-wall interfacial coefficient by an amount $\gamma_{s w}(\delta A / A)$. The area of the liquid-wall interface is similarly affected, but the solid-liquid interface tends to be smooth. Therefore the roughness changes the wetting coefficient $\Delta \gamma$ by an amount $\delta \gamma=\left(\gamma_{\ell w}-\gamma_{s w}\right)(\delta A / A)$. Since $\gamma_{s w}>\gamma_{\ell w}$, the magnitude of the wetting coefficient is thereby raised.

The effect of roughness can be described in greater detail. Premelting varies along the surface, according to the sign and magnitude of the local curvature. Enhancement of melting occurs where the geometry forces the curvature of the solid-liquid interface to be positive, as viewed from the solid side. Relative to the planar case, when the radius of curvature of the solid $r$ is large compared to the melt thickness $d$, the change in thickness $\delta d$ at spherical indentations (protuberances) in the substrate is enhanced (reduced) by a fraction that depends on the departure from bulk coexistence as

$$
\frac{\delta d}{d}=\frac{\gamma_{s \ell}}{\rho_{\ell} q_{m} t} \frac{2}{3 r},
$$

where $t$ is the reduced temperature (Baker and Dash, 1989). ${ }^{1}$

The effect can be described as a result of the GibbsThomson shift of melting temperature, discussed in Sec. I.D.2, and a complete variational treatment of the geometry of the interface from planar regions into the crevasses bears out this intuition (Rempel, 2000; Wettlaufer and Worster, 2006). As the temperature rises the interfacial melting begins in pits and scratches, then expands along clefts and the inside corners of steps. Connectivity of the liquid increases, but depending on the detailed geometry many regions remain unmelted until the tem-

\footnotetext{
${ }^{1}$ We note that Eq. (2.14) of our previous review (Dash et al., 1995) should have the undercooling in the denominator.
}

perature rises closer to $T_{m}$. In this way the melting progressively smooths the interface.

\section{Internally disordered solids}

We consider disorder as a shift of the thermodynamic state of the system relative to the ordered state. The bulk thermodynamic equilibrium between a solid $(s)$ and a liquid $(\ell)$ is characterized by the intensivity of chemical potential, which requires that $\mu_{s}(T, p)=\mu_{\ell}(T, p)$ for the locus of temperatures and pressures along coexistence. Hence, by definition, departures from coexistence take the system into the bulk region of either phase by varying the temperature or pressure or both. In the case of interfacial premelting in the absence of disorder, the long-ranged intermolecular interactions provide the field energy per molecule $\tilde{\mu}$ that shifts the equilibrium domain of the liquid phase into the solid region of the bulk phase diagram and hence $\mu_{s}(T, p)-\mu_{l}(T, p) \equiv \Delta \mu>0$. Thus, for an interfacial film of thickness $d$, the chemical potential of the film $\mu_{f}$ is

$$
\mu_{f}(T, p, d)=\mu_{\ell}(T, p)+\tilde{\mu}(d)=\mu_{s}(T, p),
$$

where, for example, in the case of nonretarded van der Waals forces a phenomenological description is given by

$$
\tilde{\mu}(d)=-\frac{2|\Delta \gamma| \sigma^{2}}{\rho_{l} d^{3}},
$$

where the parameters are the same as described earlier. Thus $\tilde{\mu}(d)$ represents a general external field energy/ particle that lowers $\mu_{f}$ relative to the chemical potential of the bulk liquid $\mu_{\ell}$.

Several possible forms of internal disorder include polycrystallinity, point defects, dislocations, and strains (Pengra et al., 1991; Beaglehole and Wilson, 1994; Netz and Andelman, 1997). The effect of disorder is to increase the chemical potential of the disordered solid $\mu_{\text {dis }}$ above the value that the relatively ordered solid would have $\mu_{s}$. If disorder lies in the neighborhood of the interface, it enhances premelting by raising the local free energy of the solid. In general terms, disorder affects premelting via an increase in the local energy density $\Delta \mathcal{E}$ (Dash et al., 2001). Hence $\tilde{\mu}=-\Delta \mathcal{E} / \rho_{\ell}$, and a state theory in which disorder is described by a quasistatic process (Dash et al., 2001) can be used to describe the effect which is to convert the solid phase to liquid at a lower temperature than in the absence of disorder as

$$
\mu_{\mathrm{dis}}(T, p, d)=\mu_{\ell}(T, p)-\frac{\Delta \mathcal{E}}{\rho_{\ell}}=\mu_{f}(T, p, d) .
$$

For dispersion forces the thickness relation is changed from Eq. (12) to

$$
d=\left(\frac{2 \sigma^{2}|\Delta \gamma|}{\rho_{\ell} q_{m} t-\Delta \mathcal{E}}\right)^{1 / 3},
$$

and for short-range forces the argument of the thickness relation is similarly modified. Therefore the film, in the presence of the external field of the intermolecular interactions, and the solid, in the presence of disorder, 
equilibrate to a state with a new thickness given by Eq. (22) above.

Equation (22) was derived in a theory of the collisional melting of ice, which occurs during the electrification of thunderstorms (see Sec. V.C.2). It may also be relevant in other situations causing local inelastic loss of energy, such as sliding on ice and snow, sintering, and collisional adhesion in astrophysical ices. At sufficiently high-energy density $\Delta \mathcal{E}$ compared to the term $\rho_{\ell} q_{m} t$ in Eq. (22) diverges, evolving from a thickened premelted film to bulk melting at a lower temperature.

\section{Impurities}

Impurities can greatly enhance premelting through a classical colligative or "solute effect" lowering the chemical potential of the solvent phase (Beaglehole and Wilson, 1994; Wettlaufer, 1999a; Doppenschmitt and Butt, 2000; Ewing, 2001). The insolubility of most impurities in ice maximizes the chemical potential difference between solid and liquid, and in the case of freezing from solution, impurities are driven from the solid, tending to form highly concentrated solution at the interface. Therefore extremely small concentrations of impurities in the parent liquid can exert major changes in surface melting, and may extend the temperature range down to the eutectic temperature.

While the experimental probing of surface melting of ice provides robust evidence for its existence, that evidence also displays the great variation in the magnitude and temperature dependence of the melting behavior [see Fig. 3 here, and Fig. 1 of Elbaum et al. (1993); Furukawa et al. (1987); and see also Beaglehole and Nason (1980)]. Hence although different experimental methodologies probe different structural aspects of the surface, the variability among experimental data obtained using the same methodology on ice motivated an attempt to gain a basic understanding of the sensitivity of surface melting to the ubiquitous presence of electrolytic impurities (Wettlaufer, 1999a). Moreover, the fact that all environmentally relevant phenomena involve contaminants in some form makes the general question of broad utility. For this reason we describe the approach in some detail, which considers the thermodynamic conditions necessary for the stable existence of an impure film disjoining a solid from its vapor, from air, from a foreign wall or between two crystal grains at temperatures below the bulk melting temperature (Wettlaufer, 1999a; Benatov and Wettlaufer, 2004). Thus this provides the underpinning of a substantial span of the environmental applications that we discuss in this review, and doubtless many others which we hope to learn about in the future.

The interfacial film is doped with an electrolyte; for example, a monovalent ionic species such as $\mathrm{NaCl}$. The missing bonds at any ice surface give rise to an increase in the Bjerrum defect density and hence the surface can be considered to have a finite charge density $q_{s}$ (e.g., Dosch et al., 1996; Dash et al., 2001). Therefore any ions present in the film will screen the Coulomb interaction with an efficiency that depends on their number density.

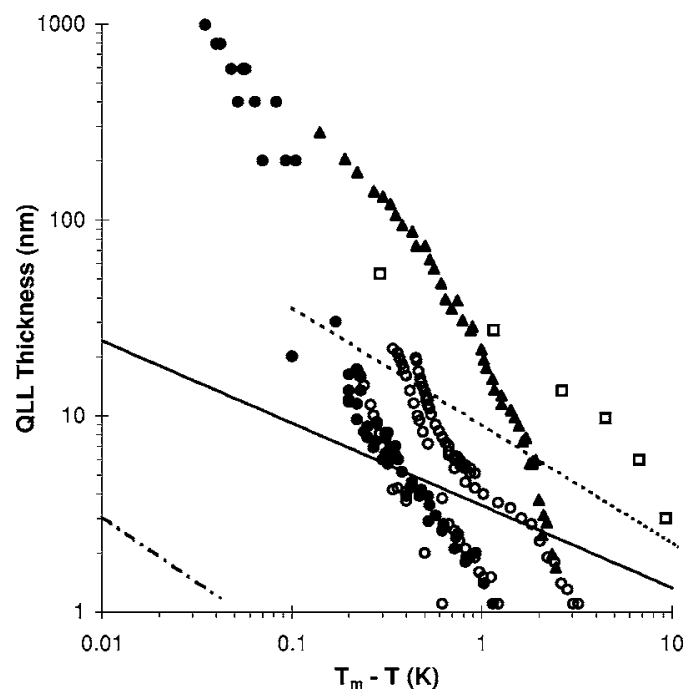

FIG. 3. Measurements of surface melting of ice, film thickness (nm) vs undercooling (K). From Pittenger et al., 2001. Solid triangles, ice in air, ellipsometry from Furukawa et al., 1987; circles, optical reflectometry from Elbaum and co-workers (Elbaum, 1991b; Elbaum et al., 1993); open circles are measurements in water vapor, and filled circles are in air; open squares are for glancing angle $\mathrm{x}$-ray scattering from Dosch et al. (1995, 1996). The solid line describes the wire regelation experiments (Gilpin, 1980c), shown in Fig. 8, the dashed line derives from experiments on ice/glass interfaces using NMR (Ishizaki et al., 1996) and the dot-dashed line is from experiment on interfacially melted ice/PVC interfaces (Wilen and Dash, 1995a). The substantial variation illustrates the great sensitivity of ice premelting to impurities and small perturbations of surface structure.

The ubiquitous influence of long-ranged van der Waals interactions manipulates the melting behavior and, as described above, their detailed influence at the surface of ice is rather rich, often requiring a complete frequency-dependent treatment (Dzyaloshinskii et al., 1961; Ketcham and Hobbs, 1969; Daniels, 1971; Knight, 1971; Seki et al., 1981; Elbaum and Schick, 1991; Elbaum et al., 1993; Wilen et al., 1995; Benatov and Wettlaufer, 2004). Ostensibly the same physical phenomena underlie Derjaguin-Landau-Verwey-Overbeek (DLVO) theory, which assesses the competition between the screened Coulomb and attractive van der Waals interactions (Derjaguin and Landau, 1941; Verwey and Overbeek, 1948). Moreover, DLVO theory has been extremely successful in describing forces that act in colloidal suspensions (e.g., flocculation), examples of which pervade technological and biological problems (Israelachvili, 1992). However, the detailed consequences in the case of ice are largely controlled by the unique complexity of frequency-dependent dispersion forces in this system and the peculiar functional dependence of the range and amplitude of the repulsive Coulomb interaction on the dopant concentration. We discuss these two effects in turn.

For the case of dispersion forces, ice presents us with three possibilities, which are discussed in more detail in Sec. II.B. There, we distinguished between the nonre- 
tarded van der Waals contribution to the total excess surface free energy per unit area, written as $F_{\mathrm{vdW}}^{\mathrm{NR}}(d)$ $=-A_{H} / 12 \pi d^{2}$, where $A_{H}$ is the Hamaker constant, or in the more phenomenological form which leads to $\tilde{\mu}(d)$ as described by Eq. (20) above. ${ }^{2}$ As noted in Sec. II.B, retarded potential effects influence the qualitative nature of melting in ice in a manner often not captured in the above formulation. Indeed, whereas the surface melting of ice has been observed to be incomplete, and grainboundary melting is theoretically predicted to be so, slight impurity doping leads to the observation of complete surface melting (Elbaum and Schick, 1991; Elbaum et al. 1993), and the prediction of discontinuous grainboundary melting (Benatov and Wettlaufer, 2004). The neglect of retardation can lead to qualitatively and quantitatively different predictions. Therefore, depending on the circumstances, it may be necessary to calculate the full frequency-dependent dispersion force response of the system $F_{\mathrm{vdW}}(d)$. This is particularly acute when long-ranged interactions, of different types, are in competition.

In a planar geometry, the screening of surface charge is described by calculating the electrostatic potential $\psi$ created by the distribution of ions of number density $n(z)$ in the film, through the solution of the PoissonBoltzmann equation:

$$
\frac{d^{2} \psi}{d z^{2}}=-\frac{\mathcal{Z} e n(z)}{\epsilon \epsilon_{o}}=-\frac{\mathcal{Z} e n_{o}}{\epsilon \epsilon_{o}} e^{-\mathcal{Z} e \psi(z) / k_{b} T},
$$

where $\mathcal{Z}$ is the valency, $z$ measures the distance normal to the plane of the film which has dielectric function $\epsilon$, and midplane ion density $n_{o}$. The free-space permittivity is $\epsilon_{o}$ and $e$ is the elementary charge. For a monovalent electrolyte, and a surface potential $\psi_{s}$ less than $25 \mathrm{mV}$, the Poisson-Boltzmann equation can be linearized in the Debye-Hückel limit which yields

$$
\psi(z)=\psi_{s} e^{-\kappa z},
$$

where

$$
\kappa^{-1}=\left(\frac{\epsilon \epsilon_{o} k_{b} T}{e^{2} n_{b}}\right)^{1 / 2}
$$

is the Debye screening length, which captures the characteristic falloff of the ion field. The bulk ion density is $n_{b}$, and $k_{b} T$ is the usual thermal energy. The repulsive force between two charged surfaces originates in the restriction of the configurational entropy of ions as surfaces are brought closer. The resulting excess interfacial free energy per unit area across a film of thickness $d$ is written

\footnotetext{
${ }^{2}$ Note that the coefficients in both approaches are related with no free parameters (Wettlaufer and Worster, 1995). Hence the Hamaker constant is related to the difference if interfacial free energies of Eq. (6) as $\sigma^{2} \Delta \gamma=A_{H} / 12 \pi$. In addition, $\Delta \gamma$ $=-\mathcal{S}$, where $\mathcal{S}$ is the spreading parameter of de Gennes (1985).
}

$$
F_{D H}(d) \approx \frac{2 q_{s}^{2}}{\kappa \epsilon \epsilon_{o}} e^{-\kappa d}
$$

where $q_{s}$ is the surface charge density associated with the Bjerrum defect density (e.g., Dosch et al., 1996).

In the classical context of DLVO theory, the Debye length is a constant, and Eq. (25) can be considered in the same sense as $F_{\mathrm{vdW}}^{\mathrm{NR}}(d)$ above. Indeed, the experimental paradigm of surface force measurements (Israelachvili, 1992) provides the film under scrutiny an "infinite" supply of solution at fixed concentration. Hence it is an experimental realization that the Debye length can be held fixed while an interfacial film thickness is varied. In contrast, during surface, interfacial, and grainboundary melting the following scenario needs to be considered. We prepare a surface at a temperature where, in the absence of impurities, a film is present. We then dope the film with $N_{i}$ moles per unit area of a single species of (for simplicity monovalent) nonvolatile impurities. Because the ice/solution equilibrium segregation coefficient is extremely small, of order $10^{-6}$ (effectively zero), and because of the low ion volatility in the case of surface melting, there are a fixed number of electrolyte ions in the film, maintaining equilibrium with the interfacial charge. Hence an increase (decrease) in film thickness is accommodated by melting (freezing) of the solid so that, up to the solubility limit, the impurity concentration is simply inversely proportional to the film volume. Therefore, at low temperatures, a surface film will thin, increase in impurity concentration, and decrease the Debye screening length: $\kappa^{-1} \propto d^{1 / 2}$.

For surface, interfacial, and grain-boundary melting, we can write the total free energy of the system as

$$
\begin{aligned}
G_{T}\left(T, P, d, N_{i}\right)= & \rho_{\ell} \mu_{\ell}(T, P) d+\mu_{i}(T, P) N_{i} \\
& +R T N_{i}\left[\ln \frac{N_{i}}{\rho_{\ell} d}-1\right]+\mu_{s}(T, P) N_{s} \\
& +\mathcal{I}(d),
\end{aligned}
$$

where $\rho_{\ell}, \mu_{\ell}$, and $N_{\ell}=\rho_{\ell} d$ are the molar density, chemical potential per mole, and the number of moles per unit area of the solvent, $\mu_{i}$ is the chemical potential of the impurity, $R$ is the gas constant, and $\mu_{s}$ and $N_{s}$ are the chemical potential per mole and the number of moles per unit area of the solid. $\mathcal{I}(d)$ is the total effective interfacial free energy and, depending on whether one is concerned with surface, interfacial, or grain-boundary melting, the construction of this function will vary (Wettlaufer, 1999a; Benatov and Wettlaufer, 2004). The entropic term $R T N_{i}\left[\ln \left(N_{i} / \rho_{\ell} d\right)-1\right]$ is written in the dilute limit of ideal solution theory, and in previous versions of Eq. (26) the $-R T N_{i}$ term was ignored because it vanishes upon minimization. Note that the analysis can proceed without the assumption of dilute solution theory but the nonideality does not change the essential behavior of the system.

We noted above that slight impurity doping leads to the observation of complete surface melting (Elbaum et $a l ., 1993)$ and hence one can capture the asymptotic be- 


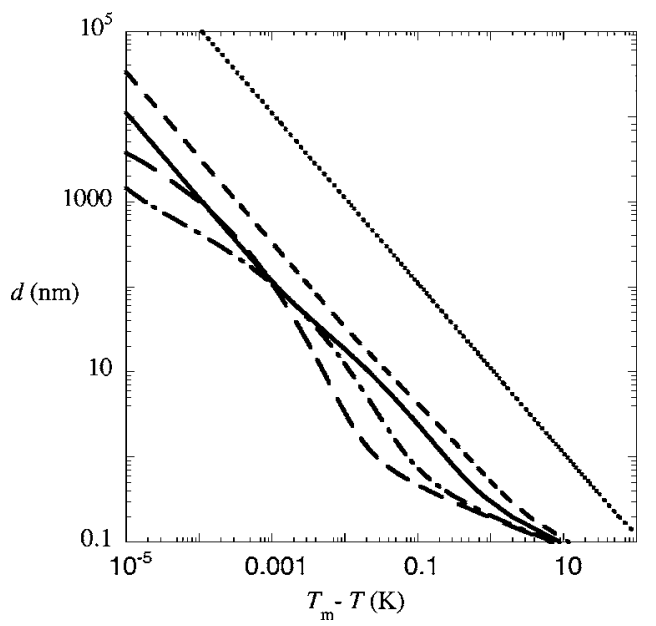

FIG. 4. Film thickness (nm) vs undercooling (K) at the interface between ice and water vapor. $N_{i}=6 \times 10^{-4}$ (long dashed line), $6 \times 10^{-3}$ (dash-dotted line), 0.06 (solid line), 0.18 (small dashed line), and $6 \mu M \mathrm{NaCl}$ (dotted line) per square meter. See the text for discussion. Adapted from Wettlaufer, 1999a.

havior of the system phenomenologically, without having to compute the full frequency-dependent van der Waals interaction $F_{\mathrm{vdW}}(d)$ from Eq. (13). Thus we write

$$
\begin{aligned}
\mathcal{I}(d) & =F_{\mathrm{vdW}}^{\mathrm{NR}}(d)+F_{D H}(d) \\
& \equiv \gamma_{d}+\Delta \gamma\left(1-\frac{\sigma^{2}}{2 d^{2}}-\frac{e^{-\kappa(d-\sigma)}}{2}\right),
\end{aligned}
$$

in which the dry interfacial energy, as noted above, is $\gamma_{d}$, and $\Delta \gamma=\gamma_{s \ell}+\gamma_{\ell_{v}}-\gamma_{d}$. The form of the second equality, in principle, allows for the extraction of the impure wetting coefficients from experiment. In contrast, for grainboundary melting the situation is more complex because the system is attractive at long range under dispersion forces and repulsive at all ranges under screened Coulomb interactions, but with an effectively rangedependent fall off and amplitude as described above. Thus it was recognized that the complete DLP theory is required for the grain-boundary and hence

$$
\mathcal{I}(d)=2 \gamma_{s \ell}+F_{\mathrm{vdW}}(d)+F_{D H}(d)
$$

is the total effective interfacial free energy. In either case, as the temperature is raised or lowered material is exchanged between the solid and liquid, and the condition for equilibrium is

$$
\frac{\partial G_{T}}{\partial N_{\ell}}=\frac{\partial G_{T}}{\partial N_{s}} .
$$

Now we present some examples deriving from these two treatments of these effective interfacial free energies.

In Figs. 3-5 below we illustrate the motivation for, and the importance of, a thorough treatment of impurity effects. The former provides the experimental evidence for the wide quantitative variation in surface melting and the latter uses the theoretical edifice described above to investigate the underlying causes.

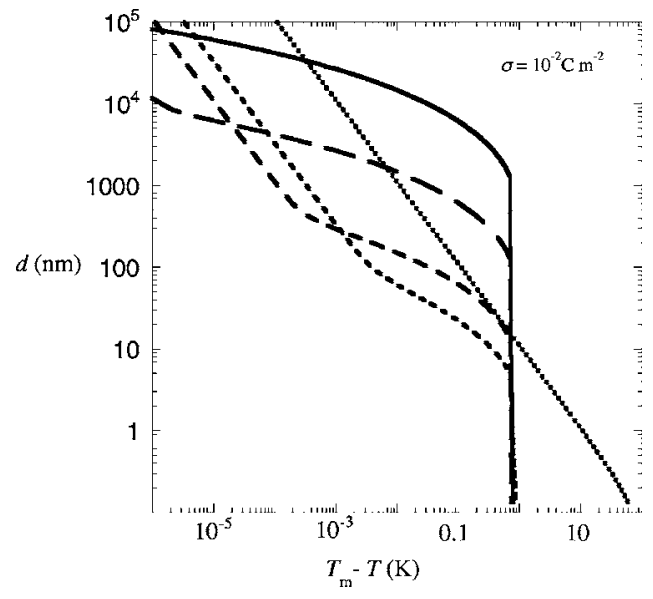

FIG. 5. Film thickness (nm) vs undercooling (K) for various monovalent electrolyte $(\mathrm{NaCl})$ concentrations at an ice grainboundary with a solid/film surface charge density $q_{s}$ of $0.01 \mathrm{C} \mathrm{m}^{-2} . N_{i}=6 \times 10^{-5}$ (solid line), $6 \times 10^{-4}$ (long dashed line), $6 \times 10^{-3}$ (medium dashed line), $1.8 \times 10^{-2}$ (small dashed line), and $6 \mu \mathrm{M} \mathrm{m}^{-2}$ (dotted line). See the text for discussion. Adapted from Benatov and Wettlaufer, 2004.

Figures 4 and 5 show the film thickness versus undercooling at the interface between ice and water vapor and another grain of ice, with different values of $N_{i}$. First, we note that for sufficiently large contamination at any interface, the entire range of melting behavior is dominated by a kind of surface Raoult's law, or colligative based freezing point depression, which is responsible for the power law $d \propto \Delta T^{-1}$. As the dopant level decreases, the situation becomes more interesting. In the vapor case, as $N_{i}$ increases, the film thickens, as expected, and the temperature at which this power law $\left(d \propto \Delta T^{-1}\right)$ begins to dominate the melting behavior decreases. The effect is particularly sensitive in the vapor case where it may overwhelm the incomplete melting controlled by van der Waals interactions in the pure case (Elbaum and Schick, 1991; Elbaum et al., 1993) and drive complete surface melting, thereby reconciling the difference between experimental observations made in different laboratories (Beaglehole and Nason, 1980; Elbaum et al., 1993; Dosch et al., 1996; Furukawa and Nada, 1997). As the temperature decreases, the first crossover to interfacial melting controlled by van der Waals interactions appears $\left(d \propto \Delta T^{-1 / 3}\right)$. For sufficiently low concentrations the range of undercooling of this van der Waals plateau is limited, and a Coulombic shoulder appears which rapidly steepens the thinning; the slope exceeding that of the purely solutal effect. The effect of impurities on the surface melting of ice is strong, but extremely subtle. Two points are worth emphasizing. First, the increasing strength of the solutal effect extends the colligative control of melting to lower temperature. Second, through its influence on the Debye length, as $N_{i}$ decreases, the range of the Coulombic interaction increases and its strength can begin to dominate that of the van der Waals interaction of a film of a higher concentration. Thus we see an interesting crossover, within the van der Waals 
plateau, between films with different values of $N_{i}$; at higher temperature, the film of larger $N_{i}$ can take a smaller thickness at the same undercooling. This type of behavior is unique to situations wherein the Debye length depends on the film thickness.

Figure 5 shows predicted effects of impurities on the grain-boundary melting of ice. Consider first the upper left-hand corner of the figure at high-temperature. The solid line has the lowest dopant level of 6 $\times 10^{-5} \mu \mathrm{M} \mathrm{m}^{-2}$. Here, the Debye length is extremely long and hence the repulsive Coulomb interaction has a longer range than the attractive van der Waals interaction; a very thick film of liquid exists at the ice grainboundary. As the temperature is lowered, the rejection of electrolytes enriches the film with ions, and hence the Debye length decreases until the attractive tail of the van der Waals force begins to come into its own. A temperature is reached where abruptly the van der Waals interaction dominates and drives a discontinuous transition to zero film thickness. The transition moves to higher temperatures as the surface charge density and dopant levels decrease thereby showing the expected behavior for melting driven solely by dispersion forces-no grain-boundary melting. Clearly, an increase in the initial dopant level reduces the Debye length. Therefore the plateau where the van der Waals and screened Coulomb interactions compete will shift to lower film thicknesses. An important aspect of these plots is that at fixed temperature the film thickness is not a monotonically increasing function of ion concentration until a threshold dopant level is surpassed. Thus here again there is a transition from melting dominated by dispersion and ionic force interactions to that dominated by the more commonly considered colligative effects and hence the bulk free energy. An exploration of variations in dopant levels and surface charge density reveals the same qualitative behavior (Benatov and Wettlaufer, 2004). Finally, at high dopant levels it is predicted that a measurable film thickness in an easily accessible range of temperature from 0 to $-10{ }^{\circ} \mathrm{C}$ exists which is the focus of present research (Thomson et al., 2005).

\section{Summary, basic consequences, and caveats regarding impurity effects}

For all ice interfaces, we find that upon addition of a small amount of impurity three different dependencies of film thickness on temperature result. Within a given type of dependence, the magnitude of the film thickness is extremely sensitive to the impurity level, and at low concentrations the Coulomb interaction can effectively compete with the van der Waals interaction at rather long range. This is especially important for grain boundaries. Figure 3 displays the variation in the magnitude and slope of the film-thickness/temperature relations and this behavior is easily bracketed by the behavior shown in Figs. 4 and 5. Therefore the results provide a reasonable explanation for the differences observed among various experimental groups as well as the changes in slope exhibited within a single experiment
(Wettlaufer, 1999b). We note that Beaglehole (1991) studied the effects of impurities on the melting of ice particles, but restricted interfacial interactions to a very short range and did not consider how the impurities extend the range of these interactions.

Although there is variability among species, their solubility in ice is generally negligible. Thus for interfacial and grain-boundary melting, impurities remain in the film, and the same is true for nonvolatile or weakly volatile species at the vapor surface. Therefore each of the interfacial coefficients in $\Delta \gamma$ will be modified in a manner that depends on, among other things, the magnitude of the surface charge density and bulk electrolyte concentration. This latter effect is due to interfacial adsorption of impurities as described by the classical Gibbs-adsorption isotherm (Schick, 1990). Thus, for example, an increase in $|\Delta \gamma|$ will enhance melting relative to the pure case, and this is demonstrated for the case of ice against air (Elbaum et al., 1993; Furukawa and Nada, 1997). We note, however, that in the case of electrolytes, the dependence of a given surface energy coefficient on concentration is complex. For example, $\gamma_{\ell v}$ increases with electrolyte concentration due to an image charge effect, which is the opposite of the case of alcohols. Thus while it is known that impurity segregation influences surface structural phase-transitions by reducing the surface energy (van der Eerden, 1993), we only have experimental evidence for the impurity dependence of the solution/vapor interface. Hence the exact dependencies of the constituents of $\Delta \gamma$ on $N_{i}$ are presently unknown to us and introduction of a more elaborate theoretical treatment is an exercise unwarranted by data. The form of our result for the vapor case (Wettlaufer, 1999a) is amenable to an experimental estimate of this effect, and the detailed sensitivity of grain-boundary predictions provides a similarly robust experimental target. In the latter case there exist no direct observations, but we note that the difficulty of direct access has recently been overcome (Thomson et al., 2005) so that we are enthusiastic about the future tests of the theory.

Finally, it is important to point out that, although we have laid bare what we think are the essential physics of impurities in surface films, there are many limitations. Israelachvili (1992) gives a thorough analysis of the limitations of Poisson-Boltzmann theory, and there are additional modifications beyond the obvious simplifications associated with the linearization, or weak-coupling limit (e.g., Netz, 2001a), that lead to Eq. (24). (i) This is a mean-field theory, and hence ion-ion correlations and discrete ion-solvent interactions are not captured; the solvent is treated as a continuous medium (Borukhov et al., 1997; Burak and Andelman, 2000). (ii) There are dispersion forces between ions and surfaces that bound them, which can be of much longer range than DebyeHückel theory would predict although not of significant magnitude at a range beyond about $3 \mathrm{~nm}$ where retardation becomes important (Edwards and Williams, 2004). For high dopant levels or multivalent ions, such ion dispersion forces can influence the static van der Waals interactions in the system significantly (Netz, 
2001b). (iii) At molecular length scales steric, or finite ion size, effects are important (Burak and Andelman, 2001). Although these phenomena may play a role in one or another regime of the predictions described, we do not expect them to be responsible for a qualitative difference in the principal features described. Perhaps even more important are other, equally distinct, effects that manifest themselves. For example, in the case of the ion dispersion phenomena, the fact that the chemical potential of the ions in ice is effectively infinite results in the film concentrating or diluting as a function of the temperature and this leads to a film thickness dependence of the Debye length and the amplitude of the interaction described by Eq. (25). Thus it is found that this leads to a long-range repulsive interaction. As for steric influences, at a grain-boundary, for example, proximity effects associated with the crystallographic mismatch will become equally important at short range. In the present experimental landscape, with the unambiguous signature of surface melting but its extreme variation between laboratories and methods, the relatively fewer and more disparate experimental approaches to interfacial melting, and the lack of a single experiment on grain-boundary melting, the simplest rigorous theory of the essential role of impurities is warranted. As the experimental picture becomes more refined, a theory that systematically includes all effects may become a compelling need. Depending on the motivations and background of the reader, such refinements and complexities may draw them into the field. Our purpose in this paragraph is to provide a roadmap to the numerous complications and limitations and we direct the reader to thorough topical books and reviews (Parsegian, 1975; Mahanty and Ninham, 1976; Israelachvili, 1992; Oxtoby, 1999).

We leave this section by highlighting the leading-order effects of impurities in films, as shown in the figures: (a) the extreme sensitivity of the film thickness to dopant level, (b) the range dependence of the relevant interactions as displayed in the temperature-dependent slopes of the various curves, (c) the "noncolligative" nature of the melting at rather low impurity level, and (d) the discontinuous transition in grain-boundary melting due to the detailed nature of long-ranged interactions in the system. We suggest that in the future rather than attempting to prepare absolutely pure samples, it is hoped that the magnitude of the impurity sensitivity can be used as a guide for future experimental work toward an approach of systematic doping.

\section{Dynamical effects}

\section{Thermomechanical pressure}

A thermomolecular pressure gradient associated with a temperature gradient produces the phenomenon known as "frost-heave" in moisture-containing porous media, e.g., frozen ground (see Sec. V.A.1). Although frost-heave is notorious in moist ground, thermomolecular pressure is a general phenomenon that can occur in any material. The basic requirement is a perturbed re-
TABLE I. The maximum thermomolecular pressure coefficients $d P_{m} / d T=\rho_{s} q_{m} / T_{m}$ for various substances, according to Eq. (33).

\begin{tabular}{ccc}
\hline \hline Substance & $T_{o}(\mathrm{~K})$ & $d P_{m} / d T(\mathrm{bars} / \mathrm{K})$ \\
\hline Argon & 83 & 5.7 \\
Gallium & 292 & 2.6 \\
Hydrogen $\left(\mathrm{H}_{2}\right)$ & 13.8 & 3.2 \\
Lead & 600 & 4.5 \\
Mercury & 234 & 7.1 \\
Water & 273 & 11.2 \\
Helium $\left({ }^{4} \mathrm{He}\right)$ & 4 & 2.4 \\
\hline \hline
\end{tabular}

gion of solid-liquid phase equilibrium, such as in Eq. (19), extending over a span of temperatures, such as thin liquid films coexisting with solid due to surface melting. There are several theoretical discussions of thermomolecular pressure from quite different viewpoints (Miller, 1978; Derjaguin and Churaev, 1980; Gilpin, 1980b; Forland et al., 1988; Dash, 1989b). Here we follow the thread of thermodynamics and fluid mechanics as done in two comprehensive reviews (Dash et al., 1995; Wettlaufer and Worster, 2006).

Referring to the discussion in Secs. II and II.C.2 we note that the interfacial term in Eqs. (7) and (19), and hence Eq. (20), is equivalent to a pressure difference $\delta P_{q \ell \ell}=P_{\ell}-P_{s}$ between the melted layer and solid. As described in Secs. II and II.C.2, this results from the thermodynamic effect of a change in the external conditions on the chemical potential of a system. The associated perturbation changes the energy per molecule by an amount $\tilde{\mu}$ that shifts the equilibrium domain of the liquid phase into the solid region of the bulk phase diagram. Hence the interfacial term introduces a pressure difference as

$$
\delta P=P_{\ell}-P_{s}=\rho_{\ell} \tilde{\mu}=\Delta \gamma(\partial f / \partial d)=\Delta \mu(T, P) .
$$

At the transition temperature $T_{m}$ of the bulk material $\Delta \mu(T, P)=0$ and hence $\delta P$ vanishes. However, the chemical potentials differ at $T<T_{m}$ as demonstrated in Eq. (8) from which we obtain a general thermodynamic relation for the thermomolecular pressure difference:

$$
\delta P=-\rho_{\ell} q_{m} t-\left(1-\rho_{\ell} / \rho_{s}\right)\left(P_{s}-P_{m}\right) .
$$

Thus a temperature gradient parallel to a surface melted interface induces a pressure gradient, which tends to drive the flow of the liquid layer.

Two limiting cases of Eq. (31) are interesting. Liquid transport ceases when the liquid layer is at uniform pressure, $P_{\ell}=P_{m}$. Solving for the pressure in the solid then yields

$$
P_{s}-P_{m}=\rho_{s} q_{m} t .
$$

Therefore liquid transport causes a pressure buildup in the solid until the flow ceases. In the case of water this maximum frost-heave pressure coefficient is approximately $1.1 \times 10^{5} \mathrm{~Pa} / \mathrm{deg}$ (see Table I). This is the maxi- 
mum pressure which can build up as a result of the flow of unfrozen water to the freezing front. When the pressure in ice reaches this value, opposed by an equal pressure from the wall, the flow ceases. The pressure exerted on ice by the wall is referred to as the overburden pressure and in natural soils is the result of the weight of ground above the ice front plus the cohesive strength of the soil. In the absence of an overburden pressure from the wall unfrozen water in the layer will flow to the ice front. It is the freezing of water to maintain the equilibrium film thickness [Eq. (12)] that is responsible for the deformation-heaving - of the wall. The pressure driving the flow in the liquid layer can be found by setting $P_{s}=P_{m}$ :

$$
P_{\ell}-P_{m}=-\rho_{\ell} q_{m} t,
$$

and we notice that the pressure decreases for decreasing temperature, causing water to flow to the colder region. The negative sign reflects the fact that the interaction between the boundary surfaces of the melt liquid is equivalent to a "disjoining pressure" which tends to thicken the liquid layer, but is opposed by the free energy of conversion of solid to liquid (see, e.g., Wettlaufer and Worster, 2006).

The most general situation prevails when the overburden pressure is between $P_{m}$ and the maximum frostheave pressure; then the pressure in the melted layer can be expressed as

$$
P_{\ell}-P_{m}=-\rho_{\ell} q_{m} t+\frac{\rho_{\ell}}{\rho_{s}}\left(P_{s}-P_{m}\right) .
$$

The essence of transport driven by this effect of intermolecular interactions is expressed as the thermomolecular pressure gradient in the melted layer:

$$
\frac{\rho_{s}}{\rho_{l}} \nabla P_{l}=\rho_{s} \frac{q_{m}}{T_{m}} \nabla T+\nabla P_{s} .
$$

The effect can occur with any force that causes premelting; it exists whenever phase equilibrium is perturbed such that liquid coexists with solid in the bulk region of the phase diagram of the latter. Liquid may exist at temperatures and pressures below the normal phase boundary for a variety of reasons: surface melting, capillarity, surface disorder, solutes, and confinement. When that occurs, a thermomolecular pressure gradient will attend a temperature gradient in the material, regardless of its constitution.

The universality of the theory has been tested by experimental studies. Several measurements of the maximum frost-heave pressure in frozen soils and other porous media have shown close agreement with the theory (Radd and Oertle, 1973; Takashi et al., 1981). In the most unusual example, thermomolecular pressures have been measured in ${ }^{4} \mathrm{He}$ solid in porous glass of several pore sizes (Hiroi et al., 1989). In these studies the thermomolecular pressure coefficient was measured at several constant pressures in the range $26-60$ bars and at temperatures above $1.3 \mathrm{~K}$. Others are shown in Table I.

\section{Flow by thermomolecular pressure}

When a system undergoing surface melting is subjected to a temperature gradient parallel to the interface, mass transport is carried by vapor as well as by the melt layer. The surface profile evolves to a nonplanar shape, as material is carried to colder regions, and continues until surface or temperature gradient conditions establish dynamic equilibrium (Dash, 1989b; Wilen and Dash, 1995a; Zhu, 2000).

The driving force for flow is illustrated nicely with reference to Eq. (30). We define the thermomolecular pressure as $P_{T} \equiv \rho_{\ell} \tilde{\mu}$. The case of relevance is when $\Delta \gamma$ $<0$ so that there is a force of repulsion (disjoining pressure) between the media bounding the unfrozen liquid film. In general we consider that the film is bounded on one side by its solid phase and our thermodynamic description requires only that it be bounded on the other side by a phase that has interactions that can be characterized. Thus the external pressure applied to the third phase $P_{3}$ is equal to that applied to the solid $P_{s}$ and this must balance the thermomolecular pressure $P_{T}$, and the hydrodynamic pressure $P_{\ell}$, giving

$$
P_{3}=P_{S}=P_{T}+P_{\ell} .
$$

As the temperature rises the film thickens and hence the thermomolecular pressure decreases and the forcebalance requires the hydrodynamic pressure $P_{\ell}$ to increase thereby driving liquid toward lower temperatures.

A clear illustration of this premelting dynamics was provided by an experimental study (Wilen and Dash, 1995a) which is compared and contrasted to other thinfilm flows in a recent review (Wettlaufer and Worster, 2006). The setup consisted of a disk of solid ice sitting on a rigid plate and covered above by a flexible membrane. A cold finger was used to cool the disk center, while the outer rim was heated to the bulk melting temperature to establish a radial temperature gradient $\nabla T$ and thereby the thermomolecular pressure between the membrane and ice increased towards the center of the disk. Since the membrane was under tension, it exerted a pressure $P_{s}$ that was proportional to the curvature of its surface (because the film thickness is much less than the radius of curvature the force exerted by the membrane was equated with $P_{s}$ ). Initially, the membrane had zero curvature so that the gradient in solid pressure vanished and the temperature gradient produced a fluid pressure gradient that caused liquid to flow from the reservoir at the disk rim and through the premelted film towards lower temperatures. In order to maintain the equilibrium film thickness, solidification occurred along the flow path, and this caused a distortion of the membrane surface. By comparing the measured evolution of the membrane height with the predictions of a complete theoretical treatment (Wettlaufer et al., 1996), it was possible to infer how the film thickness varied with tem- 
perature, assuming that the liquid viscosity was not altered by the effects of confinement.

A similar analysis for the case of an elastic capillary tube subject to an axial temperature gradient highlights the way in which dynamic effects can become localized within a finite-temperature window (Wettlaufer and Worster, 1995). At temperatures that are only slightly below $T_{m}$, gradients in the volume flux are the largest because the film is thinning rapidly and hence the thermomolecular pressure drives significant wall deformation. At much colder temperatures, deformation is limited by severe restrictions to fluid flow that are associated with reduced film thicknesses. The interplay between these regimes is changed by transients in the temperature field, a consideration that becomes important in many natural systems, as discussed further in Sec. V.

The examples just described share many similarities with other wetting problems, in which the interfacial geometry must adjust to satisfy constraints imposed by local force equilibrium. The situation is modified somewhat when interfacial premelting occurs against a rigid substrate. In such circumstances, since both ice and the substrate can support differential stresses, the forces need not be balanced locally, but a global force-balance constraint must be satisfied instead. Similar situations commonly occur in other lubrication flows, notably the classic textbook calculation of the forces required to squeeze fluid out from a thin film [see, e.g., Batchelor (1973), p. 221]. From a modeling perspective it is important to recognize that any attempt to describe the forces in such a system using local field equations implies a further degree of approximation.

As discussed in Sec. II, liquid is found at equilibrium within the solid region of the bulk phase diagram for a variety of reasons in addition to the substrate interactions that promote interfacial premelting. This can lead to confusion over whether any of these other phenomena contribute towards producing fluid flow and related dynamic effects. Moreover, the contorted interfacial geometries that are a common feature of many freezing systems make treatments requiring the evaluation of surface integrals impractical. We note that only surface interactions that cause interfacial premelting produce a force per unit area that is oriented perpendicular to the ice-liquid interface and hence capable of performing work when the interface motion continues in that same direction. By contrast, effects of surface energy, for example, produce a traction that is oriented parallel to the solid ice surface. This observation is particularly useful when considering the net force exerted over a closed surface. No matter what the surface shape, the curvature $\mathcal{K}$ integrates to zero. Therefore when premelted liquid is present because of the combined effects of substrate interactions and interfacial curvature we can calculate the net thermomolecular force as

$$
\begin{aligned}
F_{T} P_{T} d A & \approx \oint\left[\frac{\rho_{s} q_{m}}{T_{m}}\left(T_{m}-T\right)-\gamma_{s l} \mathcal{K}\right] d A \\
& =-\frac{\rho_{s} q_{m}}{T_{m}} \int \nabla T d V
\end{aligned}
$$

and the divergence theorem has been used to write the final expression on the right (Rempel, Wettlaufer, et al., 2001). This indicates, for example, that when the temperature gradient is constant, the net thermomolecular force over the surface of a substrate is simply proportional to the mass of ice that could occupy that volume. The ability to express the combined effects of surface interactions as an equivalent body force prompts an analogy with Archimedes's principle, in which the net thermomolecular force might be regarded as a form of "thermodynamic buoyancy" (Rempel, Wettlaufer, et al., 2001).

\section{COMPUTER STUDIES OF ICE PREMELTING}

Computer simulations have presented unique views of structural changes that occur during surface melting. Our brief survey begins with a molecular-dynamics study of a small crystallite during its melting process. Weber and Stillinger (1983), in their examination of a 250molecule hexagonal ice crystal, found that melting, i.e., molecular disorder and mobility, began at the surface and proceeded inward. The transition had no sharp onset; rather there was a smoothly increasing deviation from harmonic motion, beginning at $150 \mathrm{~K}$ below the melting point of the system. Although surface curvature effects play a large role in such a small system, the results evidently showed that the phase change begins with surface melting.

Kroes (1992) avoided complications of small size by studying a flat slab, modeled by 12 layers of moving $\mathrm{H}_{2} \mathrm{O}$ molecules, held in place by four layers of stationary water molecules underneath. To simulate an infinite surface, periodic boundary conditions were imposed in the horizontal directions. The molecular-dynamics simulation found the onset of surface melting of the (0001) face began with high molecular rotational and translational mobility, at about $-40{ }^{\circ} \mathrm{C}$. The discrepancy with experimental results (Beaglehole and Nason, 1980; Ocampo and Klinger, 1983; Furukawa et al., 1987; Mizuno and Hanafusa, 1987), which found that onset is much closer to $T_{m}$, is usually ascribed to limitations of the pointcharge model of the water molecule which is used. Nevertheless, this study affords a detailed view of the interlinked changes of molecular orientation and diffusion attendant on surface melting of ice.

Furukawa and Nada (Furukawa and Nada, 1997; Nada and Furukawa, 1997) applied molecular-dynamics to examine the microscopic structures of ice-vapor and icewater surfaces and their relation to growth kinetics (see Fig. 6). Their simulation showed that the surface melting of the (0001) and (1010) faces differs in onset temperature and temperature dependence of melt layer thickness, consistent with the ellipsometry measurements 


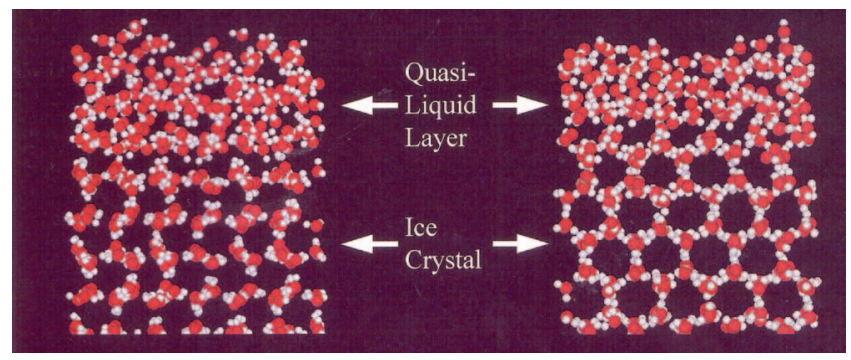

FIG. 6. (Color) Molecular-dynamics computer simulations from Furukawa and Nada (1997) show sections of ice structure during surface melting at a simulation temperature of $265 \mathrm{~K}$. The upper arrows indicate the liquid layer and lower arrows the bulk crystal. The basal plane is on the left and the prism plane on the right. Adapted from Furukawa, 1997.

(Beaglehole and Nason, 1980; Furukawa et al., 1987), discussed in Sec. IV. Recent simulations by IkedaFukazawa and Kawamura (2004) using an atom-atom potential model also find anisotropy in surface melting and argue that they are due to the enhancement of the rotational motion of water molecules driven by free $\mathrm{O}-\mathrm{H}$ bonds at the surface. Thus, in their simulations the coupling between rotational vibrations and lattice vibrations underlies the anisotropy.

The evolution from stable solid structure to mobile disordered liquid advanced layer by layer. In one highly detailed view solidlike and liquidlike regions coexisted in one molecular layer, suggesting that the evolution from solid to liquid takes place via two-dimensional phase-transitions in each molecular layer. This stepwise progress is thought to be typical of the melting of crystalline matter [see Sec. I.D.3 and Dash (2002)].

\section{LABORATORY STUDIES OF PREMELTED ICE}

\section{A. Vapor surface of ice: Surface melting}

To preface our review of laboratory studies of the surface melting of ice, we note that measurements from different laboratories differ widely; there are disagreements as to the temperature range, thickness, and functional dependence of the premelted films. The disparities may be due in part to different experimental probes, but a major cause is the great sensitivity of the ice surface to perturbations. The surface melting of all materials may be affected by variations in the atmosphere, the method of preparation, and disturbances arising from the measurement process. But the surface melting of ice is particularly delicate, due to the near balance between adhesive and cohesive forces, evidenced by its very narrow temperature range. After discussing several studies, we remind the reader of the data sets that illustrate this sensitivity as described in Sec. II.C.3.

In an early indirect study, motivated by the question of the aggregation of snowflakes, Hosler et al. (1957) performed the same type of experiment as did Faraday and quantified the force required to pull ice spheres apart: from 10 to $3330 \mu \mathrm{N}$ as the temperature rose from
$-26{ }^{\circ} \mathrm{C}$ to $T_{m}$ at equilibrium vapor pressure (see their Table I). However, the surface melting of pure, wellordered ice surfaces at equilibrium in clean environments is limited to a span of only a few degrees below $T_{m}$. Three experimental studies used optical methods (Beaglehole and Nason, 1980; Furukawa et al., 1987; Elbaum et al., 1993): the first two, ellipsometry, and the third, reflectivity at the Brewster angle. Particular care was taken to assure crystal perfection in all three, but the two older studies were conducted in air-filled chambers. Beaglehole and Nason (1980) used samples cut from a single crystal, and then annealed; Furukawa et al. (1987) studied the surfaces of "negative crystals," grown in cavities of a single ice crystal; Elbaum et al. (1993) prepared single crystals vapor deposited on freshly cleaved mica. The results agreed that surface melting depends on orientation; for example, the lowest temperatures at which Furukawa et al. could detect liquid were $-0.2{ }^{\circ} \mathrm{C}$ on the (0001) face but -2 to $-4{ }^{\circ} \mathrm{C}$ on the (1010) face. Elbaum (1991b) also found a remarkable sensitivity to the atmospheric environment. In pure water vapor, the surface melting of both the basal and prismatic planes was incomplete, with the film thickness being far larger on the former than on the latter. On basal planes, where films were thicker the melt layer increased smoothly as it was warmed, but halted a few hundredths of a degree below the bulk transition temperature at approximately $200 \AA$ after which the reflection signal showed droplets on the wetted surface with contact angle of about $0.6^{\circ}$. Thus within that narrow interval below $T_{m}$ the melted layer no longer wetted the bulk ice. The behavior was reversible on thermal cycling. It was especially notable that the general effect had been predicted; as we note in Sec. II.B, both the temperature and thickness of the incompletely surface melted film was in reasonable accord with theory where the contact angle was calculated to be $0.2^{\circ}$ (Elbaum and Schick, 1991). The incomplete surface melting explained puzzling reports we described earlier; Ketcham and Hobbs (1969) and Knight (1971) using optical microscopy, had reported water droplets on ice surfaces at temperatures slightly below $T_{m}$. Elbaum and co-workers (Elbaum, 1991b; Elbaum et al., 1993) found that the behavior changed to complete surface melting, i.e., smoothly increasing to divergence at $T_{m}$ when air was leaked into the chamber (Fig. 7). This was consistent with the earlier work (Beaglehole and Nason, 1980; Furukawa et al., 1987), which had been carried out in atmospheres of humid air, and had found that the surface melting smoothly increased all the way to $T_{m}$.

Other experimental studies of ice premelting at vapor interfaces have been carried out with a variety of techniques, including proton channeling (Golecki and Jaccard, 1978), nuclear resonance (Kvlividze et al., 1974; Mizuno and Hanafusa, 1987), adsorption isotherms (Ocampo and Klinger, 1983), x-ray scattering (Kouchi et al., 1987; Dosch et al., 1995, 1996; Maruyama et al., 2000), atomic force microscopy (Bluhm and Salmeron, 1999), sum frequency generation (Wei et al., 2002), and infrared spectroscopy (Sadtchenko and Ewing, 2002). 


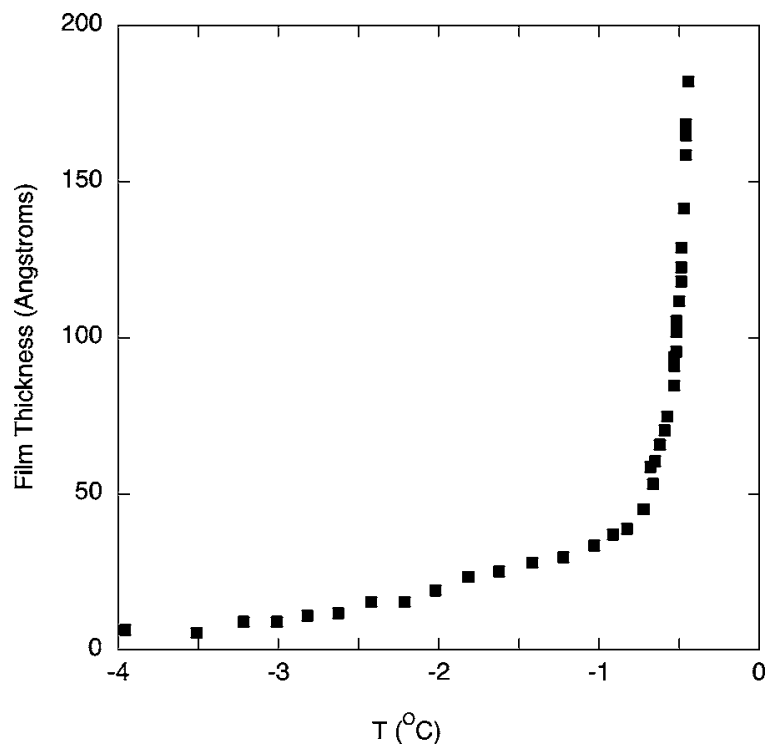

FIG. 7. Optical reflectivity measurements of the surface melting of the basal plane of ice in air. Replotted from the thesis research of Elbaum, 1991b. See also Elbaum et al., 1993.

Figure 3 compares the results of several of these studies.

\section{B. Interfaces with solid substrates: Interfacial melting}

The curves in Fig. 3 further illustrate the important differences between premelting at vapor interfaces, i.e., surface melting, and premelting at solid interfaces, i.e., interfacial melting. Many laboratory studies have explored interfacial melting at different substrates: quartz (Barer et al., 1977; Fu and Dash, 1993), metals (Gilpin, 1980a; Tsionsky et al., 2002), graphite (Gay et al., 1992; Maruyama et al., 1992), talc (Maruyama et al., 1992), glass (Furukawa and Ishikawa, 1993; Beaglehole and Wilson, 1994), silica (Ishizaki et al., 1996; Engemann et al., 2004), polystyrene (Maruyama et al., 1992), and PVC (Wilen and Dash, 1995a). A variety of techniques have been used: atomic force microscopy (Eastman and Zhu, 1995; Pittenger et al., 1998, 2001; Doppenschmitt and Butt, 2000), mercury porosimetry (Fu and Dash, 1993), quartz microbalances (Tsionsky et al., 2002), and neutron (Gay et al., 1992; Maruyama et al., 1992), x-ray (Kouchi et al., 1987; Furukawa and Ishikawa, 1993; Dosch et al., 1995, 1996; Engemann et al., 2004), and helium-atom scattering (Braun et al., 1998). Most have found that interfacial melting begins at much lower temperatures than in surface melting; instead of a range of a few $\mathrm{K}$, the span of interfacial melting may be several tens of degrees. For example, for interfacial melting against graphite, premelting could be detected as low as $-30{ }^{\circ} \mathrm{C}$ (Maruyama et al., 1992).

One of the earliest observations of interfacial melting of ice was by the technique of wire regelation, in which a wire under load sinks through an ice block (Bottomley, 1872). Wire regelation has been studied several times since then (Telford and Turner, 1963; Nunn and Rowell, 1967; Townsend and Vickery, 1967; Gilpin, 1980a). In

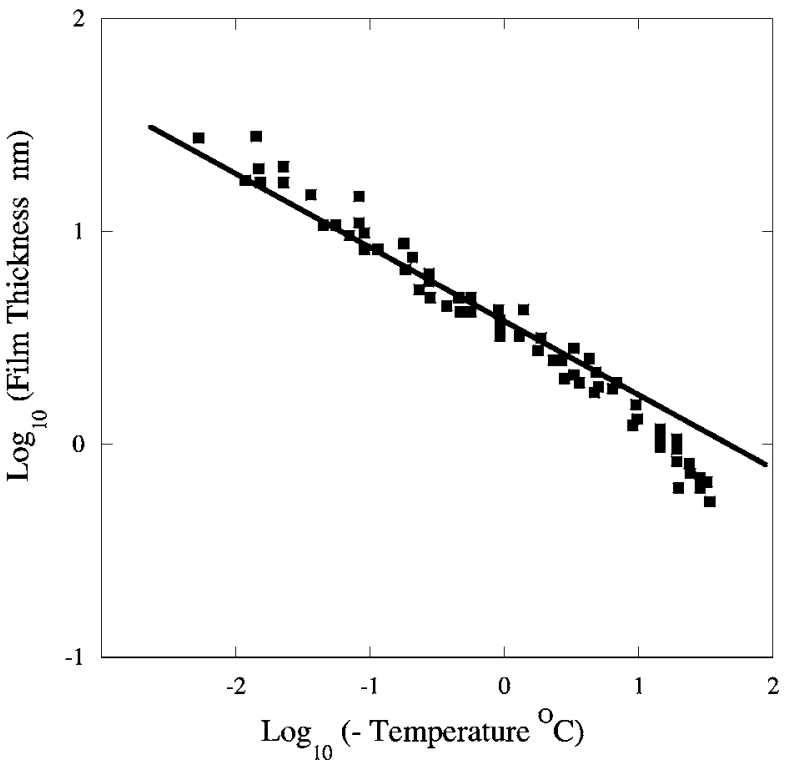

FIG. 8. The calculated thickness of water film around wires, deduced from the rate of passage of a weighted wire through ice; "wire regelation" (Gilpin, 1980c). Gilpin's data are compared with the theoretical power-law temperature dependence of thickness of interfacial melting under dispersion forces (Dash, 1989a). The more rapid decrease of the apparent film thickness at lower temperatures may be due to the effects of proximity, effectively enhancing the viscosity.

some experiments, the temperature of ice was maintained fairly close to $0{ }^{\circ} \mathrm{C}$, so that the mechanism for the motion was dominated by pressure melting. In this case ice melts under the wire, and the heat required for melting is provided by the freezing on top of the wire. The speed of passage is largely controlled by heat conduction through the wire. However, Telford and Turner (1963) found that the motion continued even when the applied pressure and temperature of the ice were too low to produce pressure melting, whereupon they suggested that there was a persistent liquid film around the wire. In 1980, Gilpin (1980a) studied the phenomenon in greater detail. He analyzed the motion, ascribed the impedance to hydrodynamic flow around the wire, and deduced the thickness of the layer by assuming that its viscosity was that of bulk water at least down to a layer thickness of $1 \mathrm{~nm}$. Dash (1989a) reanalyzed the results, and showed that the thickness-temperature data fit a 1/3 power-law, consistent with interfacial melting under dispersion forces. The comparison is shown in Fig. 8 where it is possible to see the deviation from the $1 / 3$ power-law at low temperatures, most likely caused by the loss of liquidity in very thin films.

\section{Interfaces between two crystals: Grain-boundary melting}

This section is the shortest in the review; we have deliberately singled the topic out because it represents one of the most important and least studied aspects of our subject. As noted in Sec. II.B.3, grain-boundary melting is important in the evolution of polycrystalline materials 
(Smith, 1948; Herring, 1951b; Sutton and Balluffi, 1995), for melted boundaries allow rapid transport of matter in sintering, transport, and crystal growth. We presented a detailed theory of what we expect the essential physics and sensitivity to be (Benatov and Wettlaufer, 2004) and in Fig. 2 the apparent ease with which a grain boundary can be prepared and controlled was illustrated. Although the principal optical physics of a measurement are within reach (Thomson et al., 2005), no experiment has yet directly exposed the structure of an ice grain boundary in thermodynamic equilibrium. The importance is basic to condensed-matter science and essential for an enormous range of problems in geology, geophysics, atmospheric, and oceanic sciences, which we shall demonstrate in Sec. V. We close this section by noting that a recent theoretical treatment by Style and Worster (2006) on grain-boundary grooving in the presence of surface melting provides the framework for a compelling experimental test in a more accessible geometry.

\section{Probing ice in porous media}

There is an enormous bibliography on ice in porous media, much of it involving frozen and partially frozen soil, owing to its technological and environmental importance. Regions affected by permanently or seasonally frozen soils cover a significant fraction of the Earth's land area. Their agriculture, cities, roads, and resource recovery are all conditioned by the physical properties of the frozen soil. It has been the focus of fundamental and applied research for many years; the amount and variety of this activity can be judged from a list of books, journals, and conferences cited in recent books (Johnston, 1981; Andersland and Ladanyi, 2004). We postpone discussing particular environmental effects until Sec. V; in this section we focus on the fundamental aspects explored in laboratory studies.

Basic issues concern the influence of the medium structure on the texture of the ice and liquid fraction. The primary effect of the confinement in small pores is a depression to lower temperature of the melting transition, due to the Gibbs-Thomson effect (see Sec. I.D.2). The geometry of typical porous media presents a range of surface curvature, which causes a broadening as well as a shift. Blachere and Young (1972) used porous glass as the medium; since then there have been numerous studies employing a variety of media and detection methods (Cahn et al., 1992; Maruyama et al., 1992; Belissent-Funel, 1993; Li, 1993; van Miltenburg and van der Eerden, 1993; Hills, 1994; Baker et al., 1997; Morishige, 1997, 2005; Czihak, 2000; Watanabe and Mizoguchi, 2000; Jiang, 2001; Slovak, 2001; Valiullin and Furo, 2002; Rault, 2003; Talamucci, 2003). A detailed theory of ice premelting in porous media (Cahn et al., 1992) found agreement with measurements of the unfrozen fraction versus temperature in nearly monosized powders: graphitized carbon black and polystyrene (Maruyama et al., 1992). The analysis assumes that ice undergoes complete interfacial premelting at the pore surfaces. Ice crystals at the apertures between pores are subject to the
Gibbs-Thomson effect, and the melt liquid completely wets the pore surfaces. The melting of ice is of two types: premelted films, controlled by van der Waals forces, which produce the power-law temperature dependence $d(T)=d_{o} t^{-1 / 3}$ described in Sec. II.A, and melting of the remaining bulk ice, dictated by the local curvature imposed by the pore geometry. Calculations for the two powders were made with independently determined parameters; the measured mean diameters of the powder grains and the thickness parameter $d_{o}$ obtained from analysis (Dash, 1989a) of wire regelation measurements (Gilpin, 1980a). Figure 9 shows the comparison between calculated and measured liquid fraction in both powders. Cahn et al. (1992) note that the quality of the agreement provides only a weak confirmation of the value of $d_{o}$ from regelation measurements due to the fact that the contribution of surface melting to the liquid fraction is small relative to that of the Gibbs-Thomson effect. Here we note that, having recalculated these curves, the dependence of this term in the equations on the radius of the powder particles makes the latter a sensitive fitting parameter.

New methods that have emerged from a variety of areas in soft condensed-matter physics hold promise for understanding the phase fraction in closed-packed materials over a broad range of packing fractions; the range from dilute to highly packed suspensions is of interest. In the dilute case, the role of fluid mechanical interactions between particles has been studied on a number of fronts (e.g., Leshansky and Brady, 2005), and experimentally the directional solidification of systems of hardsphere suspensions has been shown to exhibit the type of behavior found in binary alloys (Peppin et al., 2006). Highly packed suspensions occur in nature, they act as models for other industrial processes, and they provide a model setting for the investigation of proximity effects.

Significant progress in understanding the real time dynamics of such dense suspensions may be on the horizon through the use of both bright $\mathrm{x}$-ray and visible light scattering. Both methods are designed to extract information regarding the real time restricted Brownian motion of particles as yielded from the analysis of speckle patterns in transmission. In the former case, multispeckle $\mathrm{x}$-ray photon correlation spectroscopy has successfully characterized the slow dynamics of a dilute clay suspension (Bandyopadhyay et al., 2004). The method provides the dynamical structure factor from which the time constant of the aging suspension is extracted. It is hoped that at high-packing the approach will provide both the phase fraction (ratio of ice to water in the pore space) and, from the Bragg peaks, the packing geometry of the particles. The other methodology of note employs laser light and thin optical cells of the Wilen-Dash (Wilen and Dash, 1995a) variety, and it exploits the multiple scattering limit through a modification of nonergodic diffusing-wave spectroscopy (Viasnoff et al., 2002). The success of conventional diffusing-wave spectroscopy relies on the stationarity of the scattered intensity and hence the particle dynamics must be slow relative to the observation time of the speckle pattern. This must be 

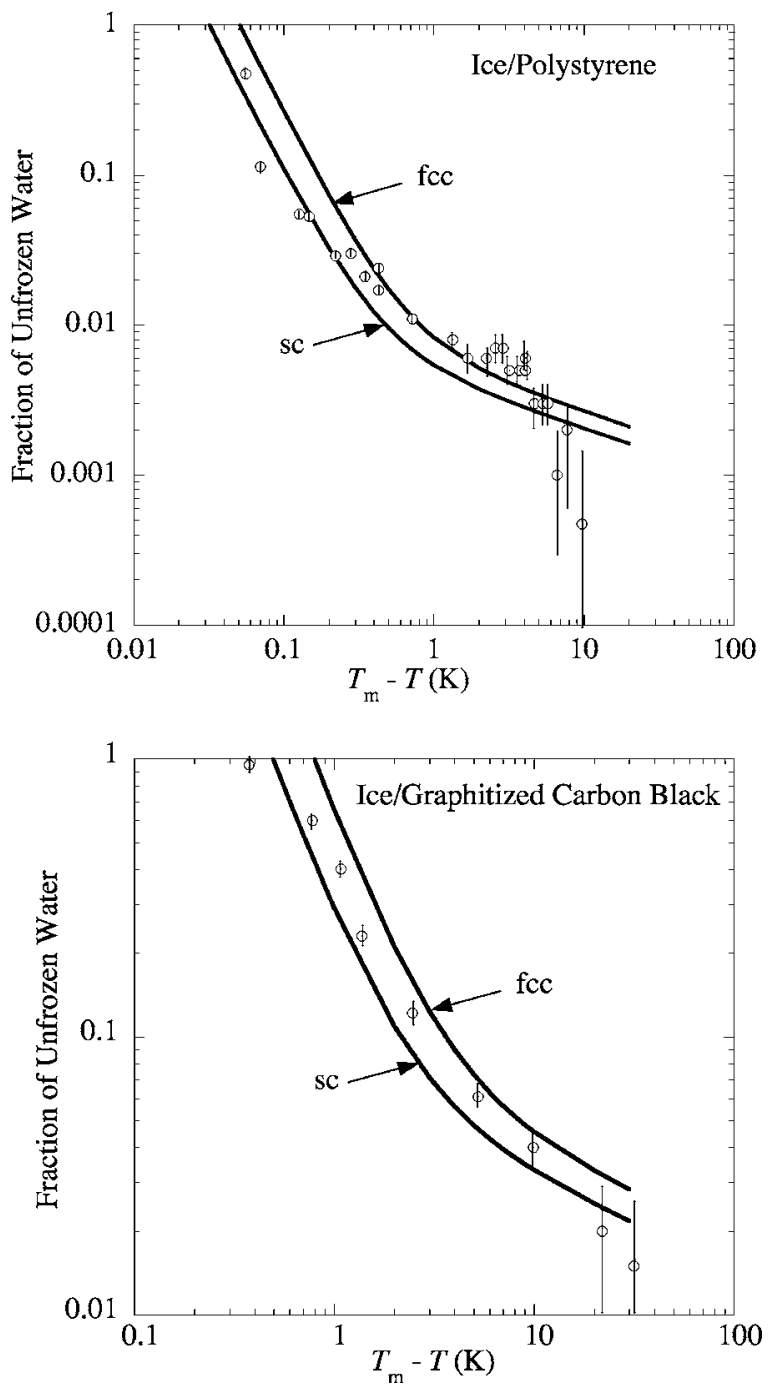

FIG. 9. Comparison between measured and calculated liquid fraction of $\mathrm{H}_{2} \mathrm{O}$ in close-packed powders. The fraction of unfrozen water is the ratio of the melted volume to the interstitial volume in the powder. The top panel shows the comparison for a powder of graphitized carbon black of average particle diameter observed in the range of $0.19-0.24 \mu \mathrm{m}$. The two curves have been recalculated using the theory of Cahn et al. (1992) and are for an assumed simple cubic (sc) and face-centeredcubic (fcc) packing of spheres using a particle radius of $0.1 \mu \mathrm{m}$, which has been used as a fitting parameter. The lower panel displays the recalculated comparison in a close-packed powder of polystyrene spheres, again for assumed simple cubic and face-centered-cubic packing of spheres, using a particle radius of $1.5 \mu \mathrm{m}$. Note that the original paper (Cahn et al., 1992) states in the text that the typical dimension of polystyrene spheres under electron microscopy is $\sim 5 \mu \mathrm{m}$ and in the associated figure caption (Fig. 3) that the average particle diameter is $10 \mu \mathrm{m}$ and that the theoretical curves are obtained with no fitting factors. We correct this here and note that the agreement shown requires the choice of the particle radius noted above.

weighed against the signal-to-noise ratio, which depends on the number of fluctuation periods required to develop sufficient statistics to generate the intensity correlation function. The system under scrutiny need also ex- hibit ergodicity in the sense that each speckle obtained measures the same distribution of light intensities as the entire ensemble of speckles over the sampling time. This is quite possibly the most serious issue in the study of the dynamics of freezing porous media and provides one of the future experimental challenges in table-top approaches. As we will see in Sec. V, such approaches are ideal for extracting quantitative information regarding the dynamics of frost-heave in real and model porous media. Thus, from the physics perspective, the quantitative unification of the dilute and nondilute dynamics of particles in partially frozen suspensions is equivalent to solving core problems in frost-heave.

\section{E. Crystal growth}

Clearly, because it is responsible for a substantial interfacial restructuring, the roughening transition profoundly influences the dynamics of molecules on the surface. An intimately related nonequilibrium transition, of particular relevance to a high-vapor pressure material such as ice, is known as the kinetic or dynamic roughening transition, which occurs when the growth rate exceeds a critical value (Cahn, 1960).

In a laboratory system, crystal growth very close to equilibrium can be achieved [see, e.g., Heyraud and Mètois (1986, 1987); Elbaum et al. (1993); and Maruyama et al. (1997)]. The principal regimes of growth are delineated in terms of the growth drive $\Delta \mu$, the free-energy difference between a molecule in the parent phase and that on the solid. At extremely small $\Delta \mu$, the accretion of mass is much slower than all available relaxation processes in the system, so that the crystal surface is indistinguishable from the equilibrium crystal shape. This "thermodynamically slow" or "shape preserving" regime has been observed at temperatures above, below, and very near the roughening transition of the prism facet of ice growing from water (Maruyama et al., 1997) and can be described via a continuous expansion of the Wulff shape (Elbaum and Wettlaufer, 1993). As $\Delta \mu$ becomes larger but, nonetheless, smaller than the activation barrier for nucleation on the facets $\Delta \mu^{*}$, the facets remain pinned and the rough orientations accrete mass to take the Wulff shape of an "equilibrium" crystal of increasing size (Elbaum and Wettlaufer, 1993). Ultimately the rough orientations grow themselves out of existence leaving a fully faceted growth form behind. If we further increase $\Delta \mu$ to a value that is above $\Delta \mu^{*}$ but below the threshold necessary to induce kinetic roughening on the facets $\Delta \mu_{\mathrm{kr}}$, the facets grow slowly by nucleation and spreading of monolayers (Wettlaufer et al., 1994).

When the growth drive is further increased so that $\Delta \mu>\Delta \mu_{\mathrm{kr}}$, the equilibrium structure of the surface cannot be maintained by the relaxation processes available to the surface. The process can dramatically change the structure of the surface and thereby the nature of the interfacial attachment kinetics [see, e.g., Cahn (1960) and Balibar et al. (1993, 2005)]. The underlying competition involved in the transition is that between a crys- 
tallization fluctuation, which may arise from the formation and subsequent decay of a subcritical surface nuclei, or a growth/melt wave induced by thermal fluctuations, and a periodic lattice potential. Because the fluctuation carries with it a latent heat the relaxation of the system requires thermal diffusion on a time scale $\tau_{T}$. All the while the interface is moving normal to itself and it takes a time $\tau_{C}$ to add a lattice plane. If $\tau_{T}<\tau_{C}$ then the heat of the fluctuation dissipates before layer completion and hence the lattice potential influences the entire history of the disturbance. In contrast, when $\tau_{T}>\tau_{C}$ the growth of the crystal is sufficiently fast that the influence of the lattice potential on the fluctuation is time periodic and hence averages to zero. Therefore the growth process itself can render the anchoring effect of the lattice ineffective, and the surface roughens dynamically. In summary, when $\Delta \mu=0$ an equilibrium form is faceted up to the thermodynamic roughening transition. However, for growth at a fixed temperature and fixed coupling strength to the underlying lattice, a facet will dynamically roughen within a range of growth drives $\Delta \mu$ $\gtrsim \Delta \mu_{\mathrm{kr}}$ where $\tau_{T} \gtrsim \tau_{C}$. Roughening is less likely in crystals with stronger intermolecular bonds. Finally, thermodynamic roughening is "smeared out" under nonequilibrium and finite size conditions, making the transition temperature less abrupt.

A qualitatively different scenario for kinetic roughening which is relevant to our subsequent discussion of collisional charging in ice (Sec. V.C.2) is manifest as follows: If the rate of step spreading is much greater than the nucleation rate, then a single layer can cover the facet before the subsequent nucleation event occurs. As $\Delta \mu$ is increased, this condition is more difficult to satisfy, and the facet becomes decorated with multiple supercritical nuclei simultaneously. But the monolayer can be completed by the spreading and coalescence of these nuclei. At still larger growth drives, the increase in the surface density of molecules increases the nucleation rate, and secondary nuclei may form on existing islands. The picture is that of islands growing upon islands and effectively roughening the surface. The process also results in a crossover from activated, or layer-by-layer growth, to continuous growth [see, e.g., Cahn (1960) and Weeks and Gilmer (1979)].

Such processes must ultimately come into contact with surface melting. When a film is present and growth is initiated the difference in the attachment kinetics between the quasiliquid/vapor and solid/quasiliquid interfaces will change the film thickness. The underlying solid may then be subject to the thermodynamic roughening transition for the solid/liquid interface or may kinetically roughen into the liquid phase. Indeed, an example of the sensitivity of ice premelting is gleaned from a study by Kuroda and Lacmann (1982). Their study explored the effects of a premelted layer on the growth rate, orientation dependence, and shape of ice crystals. They were able to correlate growth forms of distinctive crystal habits that occur in certain ranges of temperature and supersaturation with growth moderated by surface melted layers. The parameters of the melt layers were in turn derived from observations interpreted according to wettability. The melt thicknesses and temperature extend over much greater ranges than measured in the optical studies discussed above. Although the issues still need rigorous theoretical underpinning, it seems evident that the results of Kuroda and Lacmann were increased by dynamical growth effects, because both thermal gradients and kinetic roughening enhance surface melting (see Sec. II.C). A recent review treats many aspects of the role of surface physics and snow crystal morphology (Libbrecht, 2005).

\section{ENVIRONMENTAL PHENOMENA}

Where ice is found in nature, premelted liquid controls environmental phenomena to an extent that belies its tiny mass fraction in comparison to the solid. In this section we describe some of the consequences of the premelted liquid that is associated with ice in the ground, on the Earth's surface, in the atmosphere, and beyond.

\section{A. Ice on land}

Ice in the form of permafrost, which seasonally covers some $50 \%$ of the northern hemisphere, glaciers and ice sheets, which record essential clues about our past climates, and the seasonal snowpack, which is more closely coupled to weather, all involve the same basic processes at the varied interfaces involved.

\section{Frost heave}

Frost heave is the term given to the upwards displacement of the ground surface that is caused by the formation of ice within fine-grained soils. For inhabitants of temperate climates, perhaps the most familiar manifestations of frost-heave phenomena are the needle ice crystals that can be found near the surface of moist sediments on crisp winter mornings [see Fig. 10(a)]. Typically a few centimeters in height and a millimeter or so in width, these crystals often form in arrays that are capped by pebbles and small clumps of soil, which are lifted as the ice grows and draws water from the unfrozen region below. Where subzero temperatures penetrate further into porous sediments, more significant heave is associated with the formation of segregated ice lenses that are aligned parallel to the isotherms [see Fig. 10(b)]. Over time scales of weeks and months, frost action causes larger objects, such as stones and fence posts, to be pushed upwards past the surrounding fine-grained material. When left undisturbed over many seasons in polar regions, still more exotic behavior occurs-for example, causing stones to become organized in circular rings as a form of patterned ground [see Fig. 10(c)]. A complete understanding of these phenomena relies upon a quantitative description of the processes that drive premelted water towards the solidification front and determine the morphology of ice growth. 


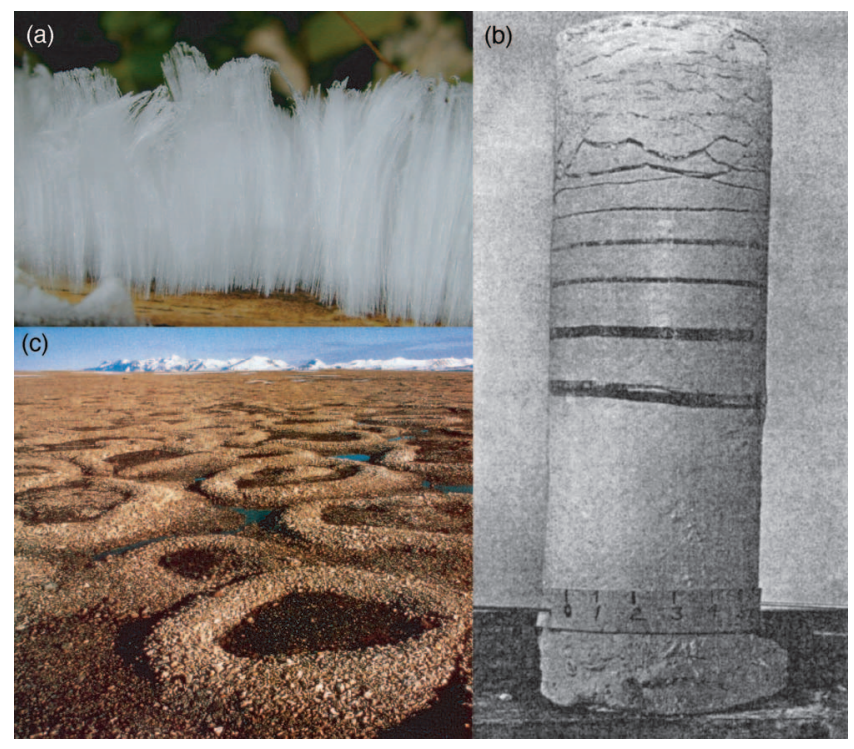

FIG. 10. (Color) Examples of frost heaving phenomena. (a) Needle ice growing out of dead wood (N. Page photo). (b) Ice lenses (dark) formed during solidification of water-saturated clay (modified from Taber, 1930, and reprinted with permission from the University of Chicago Press). (c) Stone circles in Spitspergen (B. Hallet photo, circles are 1-2 m across).

The cost of frost damage to railways and roads in northern Sweden motivated Beskow (1935) to begin the first comprehensive field investigations of frost-heave phenomena. He noted that the susceptibility of a particular soil to heaving behavior is largely determined by the particle size. Coarse-grained sands and gravels rarely demonstrate any surface deformation at all, whereas siltsized particles are highly frost susceptible, and the heave associated with fine-grained clays is comparatively slow, but capable of displacing larger loads. In North Carolina around this time, the donation of an early model commercial refrigerator enabled Taber $(1929,1930)$ to perform the first laboratory studies aimed at unravelling frost-heave dynamics. Taber recognized that the 10\% density difference between liquid water and ice is far too small to explain the magnitude of typical deformations to the ground surface. In a series of experiments, he froze benzene and nitrobenzene in porous media to demonstrate that solid lenses form and heave occurs even when the solidified pore constituents are more dense than their liquids. Indeed, more recent experiments have examined aspects of the dynamics of heave using helium (Mizusaki and Hiroi, 1995) and argon (Zhu et al., 2000) as the solidifying fluid. The magnitude of surface displacement, the total heave, is accounted for by the combined thicknesses of the solid lenses, which exclude the sediment particles as they grow. As we shall see, it is the repulsive forces associated with interfacial premelting that cause this freezing behavior.

When heat is removed from the ground surface, frostheave is driven by solidification at the base of the lowermost, warmest lens. A key question concerns the mechanism by which the liquid that supplies this growth is made to flow upwards against gravity. As Beskow
(1935) noted, the sizes of particles within frostsusceptible soils make them also amenable to having thick capillary fringes - the term given to the region of partial water saturation that extends above the water table because of surface tension effects. Everett (1961) seized upon this observation to formulate a model for frost-heave that relies on the surface energy of the iceliquid interface to explain the liquid transport to the growing lens. Recall that the Gibbs-Thomson effect (see Sec. I.D.2) describes the depression of the equilibrium melting temperature that is experienced by small ice volumes with relatively high surface areas because of the surface energy of the ice-liquid interface. In fine-grained porous media the Gibbs-Thomson effect is important for determining the relative proportions of liquid water and ice at subzero temperatures (Blachere and Young, 1972; Cahn et al., 1992; Ishizaki et al., 1996; Swainson and Schulson, 2001; Watanabe and Mizoguchi, 2001; Valiullin and Furo, 2002). It also plays a significant role in determining the permeability of the partially frozen soil to fluid flow. However, we now know that the effects of surface energy do not contribute directly to the net vertical force that lifts material and produces heave (Rempel, Wettlaufer, et al., 2001). Moreover, depending on the sign of the curvature, the Gibbs-Thomson effect can actually reduce the thicknesses of the premelted films that separate the final layer of soil particles from the lens surface and provide the conduits that supply lens growth. A closer examination of the dynamics of iceparticle interactions illuminates the underlying cause of this fluid flow.

In a creative set of experiments, Corte (1962) grew ice upwards into a bath of liquid water that contained a dilute array of mineral particles. Two distinct types of behavior were observed. For larger particles and faster solidification rates, the ice-liquid interface grew around particles and captured them within the growing solid region. By contrast, when smaller particles interacted with ice grown at lower solidification rates, particles were pushed ahead of the interface and remained nearly surrounded by the bulk liquid. Subsequent efforts to explain these phenomena have been driven by their importance to solidification dynamics in industrial and biological processes in addition to ground freezing phenomena (Uhlmann et al., 1964; Hoekstra and Miller, 1967; Bolling and Cissé, 1971; Omenyi and Neumann, 1976; Gilpin, 1980c; Bronshtein et al., 1981; Pötschke and Rogge, 1989; Azouni et al., 1990, 1997; Shangguan et al., 1992; Asthana and Tewari, 1993; Lipp and Körber, 1993; Sen et al., 1997). A comprehensive description of the underlying mechanisms was first given by Chernov and his colleagues (Chernov and Mel'nikova, 1966; Chernov et al., 1976; Chernov and Temkin, 1977), and has since been generalized and placed within the context of our modern understanding of premelting behavior (Dash, 1989b; Rempel and Worster, 1999, 2001).

Intermolecular forces that act between the particle, ice, and liquid generate a premelted film and produce a net thermomolecular force that disjoins the particle from the ice surface. The distribution of fluid pressure 
over the particle surface adjusts to produce the net force that balances this thermomolecular force and the effects (often negligible) of gravity. As a consequence, the fluid pressure beneath the particle is reduced below ambient levels and liquid is drawn through the premelted film to supply the solidification front. At low solidification rates, a steady state is established in which the rate of liquid supply is sufficient to maintain the particle at a fixed distance from the solidification front so that it is pushed ahead of the growing ice. At higher solidification rates, the resistance to fluid flow through the premelted film is too great for the liquid supply to keep pace with the rate of interface motion, and the particle is encapsulated within the ice layer. The flow paths through the narrow premelted films beneath larger particles are longer than those beneath smaller particles. This leads to an inverse relationship between the particle size and critical solidification rate that marks the transition in behavior from particle pushing to capture.

The single-particle analysis can be generalized to examine the force and mass balance conditions that determine whether ice will grow as a lens and reject sediment particles or grow around them and penetrate into the pore space below. Worster and Wettlaufer (1999) investigated the special case in which the temperature at the lens boundary is not cold enough for ice to overcome the effects of surface energy and extend into the pore space beyond the adjacent layer of particles. They found that lens growth can begin only once the temperature at the boundary is low enough that the net thermomolecular force can support the weight, or overburden, of the overlying material. If the lens temperature is decreased further, the premelted films become thinner. This causes the net thermomolecular force to increase and exceed the overburden so that the fluid pressure beneath the lens must decrease just enough that its integrated effect over the lens surface completes the force-balance. The reduction in fluid pressure causes water to be drawn towards the interface and the rate of lens growth is determined by continuity. Support for this simple description of heave dynamics comes from a series of experiments in which a water-saturated porous medium was sandwiched between two microscope slides and pulled at a constant rate through a fixed temperature gradient (Mutou et al., 1998; Watanabe and Mizoguchi, 2000; Watanabe et al., 2001). In certain parameter regimes the rate of lens growth matches the pulling speed, or freezing rate, and a single lens can grow in steady state. The theory also predicts how at higher freezing rates the fluid supply through the narrow premelted films becomes too restricted to enable the lens to grow in steady state. In experiments, at higher pulling speeds the growing lens recedes relative to the isotherms so that the temperature at its boundary decreases over time. Ultimately, the water supply to this lens may be stopped by the formation and subsequent growth of a new lens at a warmer temperature. The repetition of such a sequence results in periodic lensing analogous to that which is commonly observed in the field [Fig. 10(b)].
In order to predict the magnitude of heave, as important as it is to understand how lenses grow, it is of at least equal importance to understand how lenses are initiated. The formation of a new lens requires that the net load or effective stress supported by particle contacts must vanish when ice first begins to separate them. Miller (1978) postulated the existence of a connected network of ice that extends through the pore space beneath the growing lens in a layer known as the frozen fringe. This frozen fringe has been a key ingredient in most subsequent models of frost-heave (Gilpin, 1980b; O'Neill and Miller, 1985; Nakano, 1990; Konrad and Duquennoi, 1993). Recent neutron-diffraction studies on cohesive porous media support the hypothesis that pore ice is connected to the overlying lens (Swainson and Schulson, 2004). In their rigid-ice model, O'Neill and Miller (1985) assumed that ice within the frozen fringe moves upward with the growing lens by melting and refreezing around the matrix of soil particles. The contorted geometry of the ice-liquid interface makes it impractical to determine the net thermomolecular force directly by integrating the thermomolecular pressure over the ice surface.

Typically, an empirical weighting function has been introduced to describe how the overburden is partitioned between the pore ice, sediment particles, and pore fluid. When appropriately tuned for particular soil types, such models can reproduce the major features observed during heave. The predictive capabilities of this approach have been greatly extended by the development of asymptotic methods to aid in the solution of the model equations (Fowler, 1989; Fowler and Noon, 1993; Fowler and Krantz, 1994). It is only recently, however, that a more fundamental understanding of the factors that determine how the overburden is supported has emerged.

Recalling the discussion surrounding Eq. (37), the net thermomolecular force produced by intermolecular interactions is insensitive to the detailed shape of the iceliquid interface. The action of thermodynamic buoyancy is like that of gravity on a body in water, but here it is the gradient in chemical potential associated with unfrozen liquid that produces a force that is proportional to the mass of displaced solid (Rempel, Wettlaufer, et al., 2001). Thus when ice extends below the lens boundary to form a frozen fringe, only its volume matters-not its shape. The net thermomolecular force per unit crosssectional area is proportional to the product of the temperature gradient and mass of ice that would otherwise occupy the volume between the lens boundary and bulkmelting-temperature isotherm. With this recognized, the force and mass balance conditions are easily formulated to predict the rate of lens growth as a function of its boundary temperature for porous media with ice saturation and permeability profiles that are known as a function of temperature (Rempel et al., 2004). A regime diagram classifies the different types of freezing behavior that occur within a given soil that is subjected to different freezing rates and overburdens. At the lowest overburdens and freezing rates, steady lens growth occurs, 
without the presence of a frozen fringe. This is the regime in which needle ice grows.

With more rapid freezing and/or higher overburdens, the temperature on the lens boundary decreases, ultimately leading to the formation of a frozen fringe. At still higher freezing rates, steady growth is no longer possible and the lens recedes to colder temperatures so that the extent of the fringe increases with time. As long as the overburden is below a threshold, sufficient ice accumulates within the fringe that the net thermomolecular force acting on the pore ice beneath a particular level eventually becomes sufficient to permit a new lens to form. Over time this leads to the periodic lensing that causes the most significant heave. When the overburden is increased further, lenses can no longer be initiated and the periodic lensing regime gives way to more mundane behavior, with ice forming only in the pore space and no lens initiation or heave.

The landscape of soil-freezing phenomena holds a wealth of interesting and important questions that remain the subject of active, ongoing research. For example, examination of soil microstructures and permeability changes reveal a sensitivity to freezing history that is partly tied to frost-induced poroelastic effects (Chamberlain and Gow, 1979; Viklander and Eigenbrod, 2000) that require further study. The way in which premelting and lens formation affect solute transport in general, and contaminants in particular, is of growing concern as industrial activities further exploit the northern frontiers and global warming causes permafrost to melt (Zhang et al., 2000; Ostroumov et al., 2001). Models for the large-scale pattern formation associated with soil-freezing behavior typically rely on phenomenological or empirical rules that require further testing and refinement to be placed more firmly within a rigorous physical context (Corte, 1963; Inglis et al., 1965; Hallet, 1990; Plug and Werner, 2001, 2002; Kessler and Werner, 2003; Peterson and Krantz, 2003; Sletten et al., 2003). At much smaller scales, when freezing takes place in the tiny pores of colloidal suspensions, the assumption of a connected frozen fringe is tenuous, as high degrees of supercooling increase the likelihood for pervasive ice nucleation in distinct, separated pores. The brief overview given here should provide sufficient background to embark on more detailed studies of these and related problems. More comprehensive reviews of the early frost-heave literature are given by O'Neill (1983), Black (1991), and Henry (2000).

\section{Ice and weathering}

It has long been recognized that the formation of ice is important for the weathering of rocks and other cohesive materials (Corte, 1969; Scherer, 1993, 1999). It is perhaps somewhat surprising that the modest increase in density as water is transformed into ice is still commonly invoked in textbook discussions of weathering mechanisms, while the obvious analogy with frost-heave in unconsolidated media is often neglected. Certainly, when there is no route of fluid escape, the freezing of water in cracks and other confined spaces can generate substantial pressures. Indeed, Davidson and Nye (1985) demonstrated such an effect by freezing water in notches cut into photosensitive acrylic sheets. Nevertheless, the relative prevalence of circumstances in which the liquid phase is so perfectly confined has been questioned. Walder and Hallet (1986) give persuasive arguments for the role of water infiltration and segregated ice growth within consolidated materials by the same mechanisms as those that cause lensing during frost-heave. A simplified model of this process based on the early frost-heave model of Gilpin (1980b) has been presented by Walder and Hallet (1985). Laboratory simulations of the process are reported to compare favorably with model predictions (Hallet et al., 1991). Field investigations of bedrock fractures (Matsuoka, 2001) and the distribution of scree deposits in alpine regions (Hales and Roering, 2005) are also consistent with ice segregation as a dominant mechanism for frost damage.

It is worth noting that freeze-thaw cycles have long been considered important in soil metamorphosis and rock weathering. In the context of a modern understanding of frost damage we recognize that the mobility of premelted water is increased when the temperature approaches bulk melting. We know that the pressure gradient that drives flow is directly tied to the temperature gradient. Hence the suggested correlation between freeze-thaw cycles and weathering rates may actually be caused by enhanced segregation freezing that is promoted by the more efficient supply of fluid.

\section{Glacier motion}

The largest volumes of terrestrial ice by far are contained within the great polar ice sheets and mountain glaciers. Accurate models of glacier flow are imperative for understanding the past and current distributions of glacial ice, and for predicting the evolution of sea-level, planetary albedo, and land forms as these vast freshwater repositories respond to and influence our changing climate. The movement of glacial ice is accomplished by a combination of plastic flow, sliding, and the deformation of underlying basal sediments. It has been suggested that premelted liquid plays an important role in each of these processes.

Polycrystalline ice in bulk may be regarded as a nonNewtonian fluid, with a shear-thinning rheology in which the strain rate is proportional to the applied deviatoric stress raised to an exponent $n>1$ (Glen, 1955; Weertman, 1983; Paterson, 1994). From experiments on single ice crystals it is clear that under typical laboratory conditions deformation is most readily accommodated by the motion of dislocations along the basal plane. To maintain a space-filling configuration, the flow of polycrystalline ice with an arbitrary collection of crystallographic orientations requires at least one additional deformation mechanism to be active (Hutchinson, 1977). Whether or not this involves grain-boundary processes is a contentious issue of ongoing research and debate (Duval et al., 1983; Goldsby and Kohlstedt, 1997, 2001, 2002; 
Duval and Montagnat, 2002; Goldsby, 2006). Mounting evidence from field and laboratory studies indicates that the deformation of polycrystalline ice is indeed sensitive to grain size under conditions that are relevant for both glacial and extraterrestrial ice (Durham et al., 1997, 2001; Stern et al., 1997; Cuffey et al., 2000; Marshall et al., 2002). Moreover, the tendency for polycrystalline ice to flow more easily at higher temperatures is known to follow an Arrhenius dependence with an activation energy that increases markedly at temperatures greater than approximately -10 or $-15^{\circ} \mathrm{C}$ (Weertman, 1983; Paterson, 1994; Goldsby and Kohlstedt, 2001). This change has been attributed to the emergence of a more temperature-sensitive deformation mechanism that might be associated with an increase in water mobility along grain boundaries at higher temperatures. Further evidence for changes in the mobility of water at grain boundaries comes from the abrupt increases in grain size that are typically seen in polar ice cores at depths where such temperatures are reached (Thorsteinsson et al., 1997; de la Chapelle et al., 1998). It has yet to be conclusively demonstrated whether or not grain-boundary melting (see Sec. II.B.3) contributes to the explanation for these phenomena.

The theory of glacier sliding is founded in the work of Weertman (1957), who examined how the rate of ice sliding at the local pressure melting temperature depends on the scale of roughness elements on the glacier bed. Two primary mechanisms were considered, both in this early work and in later extensions and refinements (Nye, 1969, 1970; Kamb, 1970). For obstacles with relatively long wavelengths, ice deforms internally so that the lowermost material flow lines trace the bottom topography. For obstacles with shorter wavelengths, internal deformation would require large gradients in strain rate and make deformation more difficult. However, the ice pressure is elevated locally on the upstream side of obstacles, and this causes the melting temperature to decrease slightly so that a portion of the ice melts. At the same time, the pressure is depressed locally on the downstream side of obstacles, which promotes solidification of liquid that is supplied by fluid flow from the newly melted region. This pressure regelation mechanism is most effective for obstacles with short wavelengths because of the lower total resistance to the associated transport of liquid water and latent heat. With pressure regelation and internal deformation operating in concert, the theory predicts that obstacles of intermediate wavelength [typically $\sim 0.5 \mathrm{~m}$ (Paterson, 1994, $\mathrm{p}$. 142)] control the rate of sliding over the glacier bed. While it is difficult to access the glacier bed and directly test the validity of sliding theories, the need for further theoretical refinements is readily apparent. In its simplest form, the model just described does not predict the increased sliding rates that are often observed to correlate with enhanced melt-water supply and the corresponding pore pressure increases that are a seasonal feature in many glacial settings (Zwally et al., 2002; Joughin et al., 2004). Neither does it treat the frictional stresses that are exerted by entrained sediment particles, which have been invoked as an important contributor towards the overall shear stress at the bed (Boulton et al., 1979; Hallet, 1981; Iverson et al., 2003; Cohen et al., 2005). Model extensions, based on Gilpin's (1979) treatment of a liquidlike layer separating the ice from the bedrock, enabled Shreve (1984) to predict the slow sliding of glaciers that are below the bulk melting temperature at their beds. Sliding at temperatures as low as $-17^{\circ} \mathrm{C}$ has since been observed in the field (Cuffey et al., 1999). Further efforts are needed to develop a self-consistent theory for glacier sliding that proceeds from a modern understanding of premelting behavior to include the mechanical and thermodynamic constraints on the thickness and fluid pressure of the liquid films that disjoin ice from entrained particles and the glacier bed.

Outlet glaciers and ice streams are the primary conduits responsible for draining the vast ice sheets of Greenland and Antarctica. These rivers of ice are characterized by their comparatively rapid flow rates, low surface slopes, and the presence of deformable till at their beds (Paterson, 1994). The rapid motion is accomplished by a combination of sliding and sediment deformation-plastic flow of ice itself is of secondary importance. The activity of ice streams is known to be transient on time scales ranging from days (Bindschadler, King, et al., 2003; Bindschadler, Vornberger, Joughin, et al., 2004) to centuries (Conway et al., 2002; Bougamont et al., 2003; Ng and Conway, 2004). Models of ice stream flow highlight the role of pore fluid pressure in controlling the effective stress that resists deformation of the glacial till (Kamb, 1991; Raymond, 2000; Tulaczyk et al., 2000). Schoof (2005) questioned the physical underpinning of the most commonly used parametrizations of sliding for both fast flowing glaciers and ice sheets. One consequence is that the classical lubrication approximation for the ice flow will then fail when water pressures at the bed are high and hence the appropriate sliding laws are more akin to those found in solid friction rather than in hydrodynamic lubrication.

A complete treatment of the basal hydrologic system must include the presence of premelted liquid along the ice-till interface. The hypothesis that ice-till interactions may be related to the lens-sediment interactions that occur during frost-heave (Christoffersen and Tulaczyk, 2003) has been bolstered by the visual similarities revealed by bore-hole camera observations of sediment layers incorporated into the basal regions of ice streams (Carsey et al., 2002). It should be emphasized that the normal stress imposed by the ice weight has little dynamic effect in such systems, since this merely sets the temperature of the bulk melting isotherm. The pore pressure within the premelted liquid films that disjoin the glacier bed from the underlying till is determined by a force-balance condition that involves only the overburden associated with deviations from hydrostatic pressure in the ice. This overburden is imposed by a combination of the weight of sediment incorporated within ice and dynamic effects of bed-normal flow. Efforts to incorporate frost-heave dynamics into descriptions of basal conditions that permit ice stream flow are at an early stage 

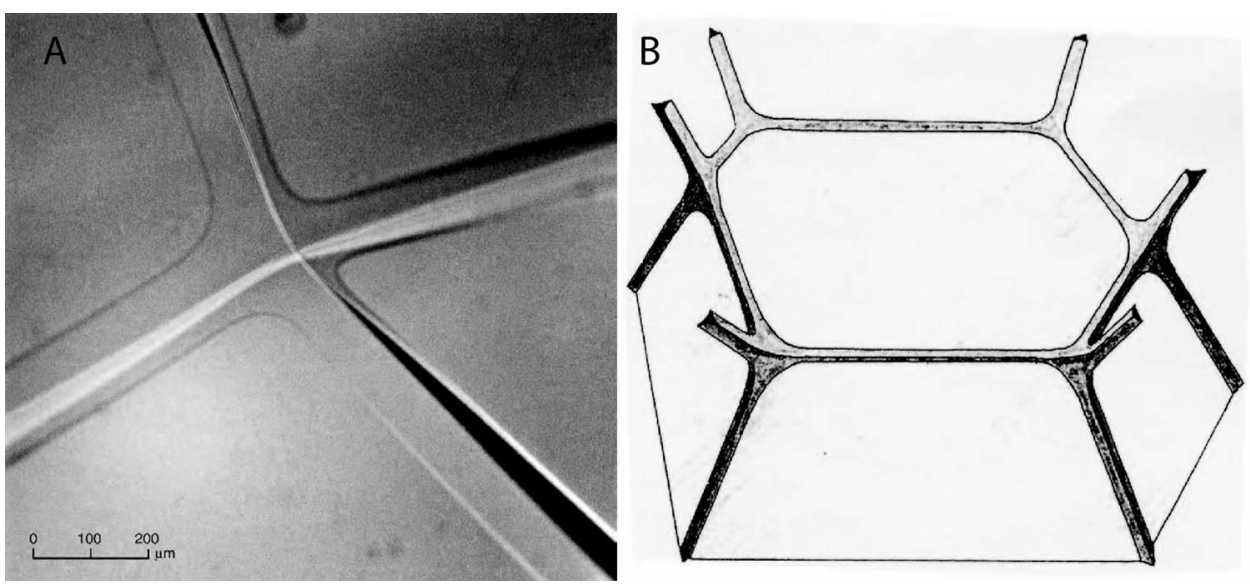

FIG. 11. (a) The intersection of four liquid veins in polycrystalline ice (H. M. Mader photo, published originally in Mader, 1992a). (b) Schematic diagram of the interconnected vein-node system (from Nye, 1989, where it was modified from Smith, 1948). Reprinted from the Journal of Glaciology with permission of the International Glaciological Society. of development. It remains to be seen how the role of frost-heave compares with those of the other mechanisms that are thought to cause sediment entrainment (Iverson, 1993, 2000; Iverson and Semmens, 1995; Alley et al., 1998, 2003).

\section{Veins and nodes in polycrystalline ice}

In polycrystalline materials that are near their melting temperatures unfrozen liquid is present in equilibrium with the solid at the nodes that mark the closest approach of four-grain intersections (Smith, 1948). For ice, a dihedral angle of less than $60^{\circ}$ is found between adjacent ice-water surfaces as they approach the line of points where two crystals contact (Walford and Nye, 1991). This implies that the overall surface energy is minimized when liquid fills veins along triple junctions where three grains meet and these veins join the nodes to form a connected network (Nye and Frank, 1973; Nye, 1991a, 1992) (see Fig. 11). Laboratory experiments confirm that the geometry of the liquid network takes this predicted form (Mader, 1992a, 1992b), which is homologous to the plateau borders in a foam. Viewed from the liquid, the vein walls are convex, with radii of curvature that decrease as the temperature falls further below the bulk melting temperature. The latent heat associated with the presence of unfrozen water is compensated by a combination of the effects of the solid-liquid surface energy and the reduction of the chemical potential of the liquid produced by dissolved impurities. The surfaceenergy effects are typically only important very close to bulk-melting conditions, and impurity effects are thought to control the overall liquid content at colder temperatures (Nye, 1991a).

In other terrestrial materials, the vein network has long been considered as an important potential conduit for material transport (Frank, 1968; Bulau et al., 1979; Shankland et al., 1981). Theoretical models for water flow through the veins of temperate glacial ice (Lłiboutry, 1971; Shreve, 1972; Fowler, 1984) suggest that this mode of englacial transport typically represents only a minor contribution to the total fluid flux, which is dominated by flow through moulins, channels, and fractures (Röthlisberger, 1972; Paterson, 1994; Fountain et al., 2005). Nevertheless, phase equilibrium conditions along vein walls are thought to represent an important buffer in controlling the temperature within glacial ice (Harrison, 1972). When placed adjacent to a pure liquid source, the rate of vein growth is in excellent agreement with model predictions based on the transport of heat and impurities that are dissolved within an idealized vein network (Nye, 1991b). However, observations of the liquid contained within natural glacial samples reveal the presence of liquid lenses along two-grain boundaries and suggest that flow through the vein network itself may often be impeded by the presence of bubbles (Raymond and Harrison, 1975). The liquid lenses are not thermodynamically stable under hydrostatic conditions. Laboratory experiments have produced lenses under moderate deviatoric stresses, but the conditions of their formation and evolution are not fully understood (Nye and Mae, 1972). Deep below the surface of the great ice sheets, where bubbles are no longer present and deviatoric stresses are low, the vein network is expected to conform more closely to the idealized foamlike case [see Fig. 11(b)]. Flow through the vein network is still expected to be relatively slow because of the small vein cross sections that can be supported by typical impurity loadings and temperature conditions. Very near the glacier base, where the temperature warms towards the bulk melting point, the permeability of the vein network increases to a level where fluid flow may become important, for example, in regulating material exchange with subglacial lakes (Rempel, 2005).

\section{Ice core climate proxies}

The record of ancient atmospheres lies deep in Greenland and Antarctic ice, and samples extracted by coring are being read today. The record was originally written in and on snow, by the adsorption of impurities on snowflakes as they drifted down through the atmosphere and by continued adsorption as it lay close to the surface. ${ }^{3}$ Then, with further snowfalls, compaction of the lower layers closed the channels and trapped the air in each layer. By seasonal variations and occasional telltale

\footnotetext{
${ }^{3}$ The oxygen isotopic ratio is trapped within and on ice rather than adsorbed in the strict sense of the term.
} 
signals such as ash from volcanic eruptions, the age of a layer can be determined. Thus the composition of the atmosphere during that early snowfall and the date of its deposit might be obtained by analysis of such a core. Until recently, it was assumed that the trapping of the air channels automatically trapped the impurities as well, but recent studies have called the assumption into question (Rempel, Waddington, et al., 2001). It has been suggested that over long time periods an offset may develop between the depths of the impurities and ice with which they were originally deposited. Also, there are quantitative uncertainties in the adsorption of certain chemicals on ice and snow, so that it is not clear to what extent samples are an accurate measure of their concentrations in the original atmosphere.

Research into the adsorption of gaseous impurities on ice and snow has been carried out in laboratory studies by many groups (Clapsaddle and Lamb, 1989; Valdez et al., 1989; Mitra et al., 1990; Conklin and Bales, 1993; Conklin et al., 1993; Chen and Crutzen, 1994; Tabazadeh et al., 1999; Domine et al., 2000; Huthwelker et al., 2001, 2004; Cho et al., 2002; Boxe et al., 2003; Khvorostyanov and Curry, 2004; Sadtchenko et al., 2004). Particular emphasis is on the uptake of $\mathrm{SO}_{2}$, which is a significant component of volcanic emissions and of the combustion of fossil fuels. These studies show that the uptake can be substantially greater than the amounts that can be adsorbed by dry solid surfaces, but are consistent with solution in liquid layers such as due to premelting. The effect depends on temperature and concentration; for example, measurements showed effectively dry adsorption at $-30^{\circ} \mathrm{C}$, but liquidlike sorption at $-8^{\circ} \mathrm{C}$ (Conklin et al., 1993). The relatively low temperature of premelting must be ascribed to the enhancement of surface melting by the chemical itself (see Sec. II.C.3).

The trace constituents extracted from Greenland ice cores provide continuous, high-resolution records that extend back more than 100000 years (Dansgaard et al., 1993; Severinghaus et al., 1998). The much slower accumulation of snow in Antarctica has helped to preserve clues to the history of climate change over the past 800000 years (Petit et al., 1999; Epica Community Members, 2004). Following the closure of air passages that connect with the glacier surface, for most of these extended residence times, alterations to the distribution of trace constituents depend critically on exactly where each species resides in relation to the boundaries of grains. Many of these constituents are soluble impurities that cannot fit easily within the crystal structure of ice, and are expected to be found at elevated concentrations within liquid-filled veins and nodes (Wolff, 1996). The manner in which the electrical properties of polycrystalline ice depend on temperature is consistent with this view (Wolff and Paren, 1984; Fujita et al., 2002). More direct evidence has come from energy-dispersive $\mathrm{x}$-ray microanalysis of veins containing $\mathrm{H}_{2} \mathrm{SO}_{4}$ (Mulvaney et al., 1988; Wolff and Mulvaney, 1988), and Raman spectroscopy of veins containing both $\mathrm{H}_{2} \mathrm{SO}_{4}$ and $\mathrm{HNO}_{3}$ (Fukazawa et al., 1998) in ice from Antarctica. Although the eutectic temperatures for these acids are extremely low $\left(-75{ }^{\circ} \mathrm{C}\right.$ for $\mathrm{H}_{2} \mathrm{SO}_{4}$ and $-43{ }^{\circ} \mathrm{C}$ for $\left.\mathrm{HNO}_{3}\right)$ and their presence in the veins comes as little surprise, questions have been raised as to how prevalent they are in solution under in situ conditions. It is difficult to resolve the issue of "in situ detection" due to the fact that existing methodologies radically alter the ice samples during the measurements themselves. Several studies have made use of energy-dispersive x-ray microanalysis to image the surfaces of ice samples undergoing rapid sublimation within a scanning electron microscope (SEM) cold stage. Impurities are found concentrated both in mobile filaments that appear to trace the grain boundaries and at isolated spots on sublimating surfaces that were originally in the grain interiors (Cullen and Baker, 2001; Barnes et al., 2002; Baker and Cullen, 2003; Baker et al., 2003; Barnes, Wolff, Mader, et al., 2003). In a recent study using Raman spectroscopy, only micron-sized inclusions of various salts were found, and mass balance arguments suggested that these could account for something like half of the $\mathrm{SO}_{4}{ }^{2-}, \mathrm{Na}^{+}, \mathrm{Mg}^{2+}$, and $\mathrm{Ca}^{2+}$ detected by subsequent ion chromatography of the melted samples (Ohno et al., 2005). Observations such as these highlight the importance of efforts to better understand the structure of grain boundaries (see Sec. II.B.3) and the chemical interactions that occur within ice (Fisher, 1987; Landais et al., 2004).

Idealized models are useful for exploring how postdepositional changes can be expected to affect the ice core records. Because compositional diffusion through the solid ice grains themselves is extremely slow, efforts have concentrated on determining whether diffusion through vein liquid might effectively short-circuit this process. Variations in climatic conditions at the time of deposition produce large spatial variations in the bulk concentration of impurities that are eventually measured in melt-water from the core, that is, the mass of impurities divided by the total mass of polycrystalline ice. However, the intergranular concentration of impurities that are dissolved in veins, that is, the mass of impurities divided by the mass of premelted liquid, must be in equilibrium with the solid vein walls. Hence, under in situ conditions the intergranular concentration is expected to change only with gradual increases in temperature that result primarily from geothermal heating at depth. This implies that changes in the bulk concentration should correlate with changes in the total volume of premelted liquid that is present. Treating the polycrystalline ice as a chemically reacting porous medium, the effective diffusivity that describes the rate at which soluble impurities move through the liquid is equal to the product of the molecular diffusivity and the local liquid volume fraction. One predicts that transport is more (less) rapid where the bulk concentration is higher (lower), and the intergranular concentration changes only slightly over the short length scales on which large changes in bulk concentration occur. A wavelike behavior results; a net accumulation of impurities occurs at places where the transport rate varies from fast to slow and a depletion in places where the transport rate increases from slow to fast. Since the maximum rate of accumulation is exactly 
$90^{\circ}$ out of phase with the maximum rate of transport, the signature effect of diffusion is not to smooth the bulk impurity record, but rather to translate it slowly towards warmer temperatures (Rempel, Waddington, et al., 2001). The predicted behavior is similar to that observed in ice containing isolated brine pockets, which also slowly migrate towards warmer temperatures (Wettlaufer, 1998). Such anomalous diffusion can lead to an offset between an impurity record and ice with which it was originally deposited, thereby affecting interpretations for the relative timing of different climatic events. The good news for paleoclimate reconstructions is that, because intergranular concentration gradients are so shallow, these changes are expected to be significant only on relatively long time scales. For example, the predicted offset is approximately one century for the 100000 year-old ice in the cores recovered from the summit of Greenland (Rempel, Waddington, et al., 2001). Since abrupt climate change has occurred in the past on time scales much shorter than one century, this emphasizes the importance of postdepositional effects on the high-resolution interpretation of records stored in ancient ice.

Postdepositional diffusive alterations are complicated somewhat by competing effects of different impurity species that are dissolved together in the veins. Gradients in intergranular concentrations of the various species cause the bulk concentrations to adjust until the dissolved species vary in phase with each other (Rempel et al., 2002). Such behavior is similar to that which had previously been interpreted to result from the formation of insoluble salts (Pasteur and Mulvaney, 2000). Diffusive rearrangements of this kind are expected to be relatively rapid, with subsequent alterations characterized by the slow signal migration described above (Rempel et al., 2002). When a significant fraction of impurities are segregated from the vein liquid, the speed of these predicted changes is reduced (Rempel and Wettlaufer, 2003b). Prolonged signal alterations can result if the vein impurity content varies sufficiently to include regions where effects of surface energy become significant in affecting the liquid content. This has been suggested as a mechanism for broadening the acidic peaks associated with volcanic eruptions (Barnes, Wolff, Mallard, et al., 2003).

Premelted liquid is expected to affect the fate of other types of trace constituents including biota (Campen et al., 2003; Warren and Hudson, 2003) and oxygen isotopes. For example, temperature variations are recorded in the ice cores as changes in the ratios of the stable isotopes of oxygen and hydrogen. Though most of the water is contained within the solid ice grains, the vein network can nevertheless have an important influence on the rate at which isotopic records are smoothed by diffusion. Johnsen et al. (1999) reported that the isotopic signals in the Holocene ice from central Greenland suggested a diffusion coefficient an order of magnitude greater than that measured in ice single crystals. Nye (1998) formulated a model consistent with such a rate of alteration, by assuming that diffusion across the ice grains to vein boundaries controls how rapidly the overall signal can change. Johnsen et al. (2000) proposed an alternative model in which the isotopic content of the ice grains and vein liquid are assumed to be locally well mixed, but diffusion of the isotopic anomalies is limited by the small liquid fraction available. By treating the diffusion across the ice grains and the diffusion through the vein liquid together, one recovers each of these models as opposite end members (Rempel and Wettlaufer, 2003a). Under conditions typical of the Greenland cores, the presence of veins is predicted to enhance the rate of isotopic diffusion by approximately a factor of 3 . It has since been suggested (Johnsen, 2005) that another explanation for more rapid changes inferred from central Greenland might be an increased rate of vapor-phase diffusion associated with warmer local temperatures shortly after the snow was deposited.

\section{Metamorphosis of snow}

Scientific curiosity is by no means the most common motivation for making a snowball. Yet, the search for a deeper understanding of the mechanisms of sintering in snow has been at the heart of investigations into the structure and phase behavior of ice since the time of Faraday (1850) (see Sec. I.C). The microstructures of snowfields strongly influence their longevity, mechanical properties, permeability, and reflective characteristics. Studies of snow metamorphosis are driven by the importance of these changes to fields as diverse as ice core climatology (Herron and Langway, 1980), watershed resource management (Foster et al., 2005), avalanche hazard assessment (Schwizer et al., 2003), and recreational equipment design (Colbeck, 1991). Snow grains rapidly lose their depositional forms upon settling, with their edges becoming rounded to reduce the total surface energy. Subsequently, the average grain size increases and intergranular bonds evolve as material redistribution is accomplished by a variety of transport mechanisms, including vapor diffusion, surface diffusion, bulk diffusion, solid-state deformation, and grain-boundary diffusion. To determine which of these is most important in a given circumstance, a common strategy involves observing the time dependence of snowfield metamorphosis and comparing with predictions of quantitative models.

It is widely known that the characteristics of snow strongly depend on whether it is "wet" or "dry." At one extreme, the addition of rainwater to snowpacks induces rapid density increases and can trigger avalanches (Conway and Raymond, 1993; Marshall et al., 1999). Even without such additional liquid input, wet snow is at the bulk melting temperature so that macroscopic quantities of the liquid, solid, and vapor phases coexist. Under these conditions, cohesion drops rapidly as the liquid content increases to a level where there is more water than can be accommodated in pendular rings around grain contacts (Colbeck, 1979). As the temperature is decreased below the bulk melting temperature, there is a monotonic reduction in the quantity of premelted liquid that coats the grain surfaces and potentially the grain 
boundaries as well. In such dry snow, the grain shapes and strength of intergranular bonds are sensitive to the rate at which they grow. For example, when the temperature increases with depth in the snowpack, gradients in the equilibrium vapor pressure drive upwards mass transfer. If the transport rate is sufficiently low, the surface energy is minimized by maintaining rounded grain surfaces as they coarsen. For higher transport rates, attachment kinetics become rate limiting and faceted grains are formed, with much weaker intergranular bonds (Giddings and LaChapelle, 1962; Colbeck, 1982; Sokratov, 2001). Much has been learned about snow metamorphosis through applying models that are also appropriate for describing the sintering of amorphous materials, such as glass beads. Recent efforts to describe changes in the strength of intergranular bonds in dry snow have departed from this tradition by focussing attention on the role of grain-boundary diffusion and evolving grain-boundary grooves at intergranular contacts (Colbeck, 1998). A key uncertainty in these models is the appropriate value of the product of grainboundary diffusivity and grain-boundary thickness. SEM observations of slight ridges at the grain contacts had been used to argue for an extremely high mobility for the water along grain boundaries (Adams et al., 2001). However, evidence that these ridges might actually represent localized impurity concentrations in an unknown state of hydration casts doubt on whether such observations actually provide any meaningful quantitative constraint (Barnes, 2003).

\section{B. Ice on the oceans}

On a given day one of the polar oceans is freezing to form sea ice that reflects radiation and insulates the ocean below. The areal extent of ice changes seasonally over many millions of square kilometers thereby mirroring the polar night. In the northern hemisphere ice is exported into the North Atlantic Ocean where it melts and influences the stability of the water column and large-scale ocean circulation, making oceanic solidification the primary distillation process in the high latitudes (Aagaard and Carmack, 1994). Some of the oldest questions regarding the role of sea ice and climate persist unanswered; the most serious one being: Will we always have ice in the polar oceans? In a more immediate setting, we ask about the ecosystems of the polar regions and to what extent are the pollutants that infest freezing waters trapped in the ice and transported? A plethora of basic questions concerning the growth and decay of sea ice bear strongly on these and other geophysical and biological phenomena.

There is a particular question that captures the attention of the physically minded: What controls the dynamical phase behavior of the ice that covers the ocean? The fact that sea ice is much less salty than the ocean from which it has formed has been appreciated ever since the material has been walked upon. A more subtle issue deals with the fact that sea ice does have a finite fluid and hence salt fraction and this evolves in space

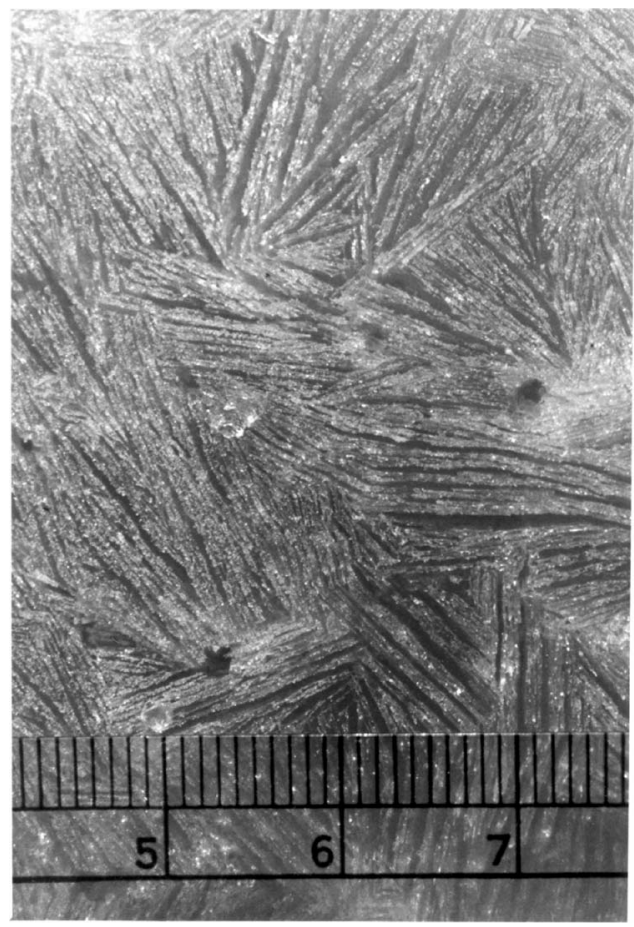

FIG. 12. The underside of the laboratory-grown sea ice showing the cellular solid/liquid structure of the interface and two clear brine-drainage channels. The $c$ axes of the ice crystals are oriented principally horizontally, parallel to the plane of the page, displaying the columnar structure and permeability of natural sea ice. The large-scale is in $\mathrm{cm}$. Adapted from Wettlaufer et al., 1997.

and time throughout the material. The bulk salinity influences the bulk thermal properties of the material and this fact was an essential ingredient in the earliest thermodynamic models of sea ice (Untersteiner, 1968). The origin of the phase behavior is essentially identical to that of any directionally solidified alloy. Because salt is rejected by the ice lattice there is a build up of a solute enriched boundary layer adjacent to a solidification front [see, e.g., Nagashima and Furukawa (1997)]. Due to the local interfacial depression of the freezing point caused by this buildup, the boundary layer becomes supercooled; the actual temperature is less than the solute determined freezing point, so-called constitutional supercooling. This allows interfacial corrugations to grow and create a cellular interface (Fig. 12). The conditions of natural growth are always such that this instability is operative and hence sea ice will grow as a two-phase, two-component material, making it among the most dynamic effective media of all terrestrial ice forms (comprehensive reviews include Weeks, 1998, and Wettlaufer, 1998). Such materials are generally referred to as mushy layers and they occur during directional solidification in many binary systems ranging from metallurgical to geological (Worster, 1997).

The lower bound on the spatial scale of the cellular substructure is dictated by interfacial energy, which controls the local freezing point at the surface through the Gibbs-Thomson effect. Moreover, the intrinsic aniso- 
tropy which characterizes the fabric of the matrix is produced by the sensitive dependence on crystallographic orientation of the attachment of water molecules (Wilen and Dash, 1995b; Dash et al., 1999). Because the substructure is grown into sea ice, both its bulk thermal and transport properties owe their origin to these microscopic effects. One such macroscopic property is the permeability, which describes the resistance to flow of brine through the porous sea ice matrix. The importance of the permeability is great; it underlies the process that determines the rate at which dense brine is delivered to the surface of the polar oceans. The essence of the process is as follows: ice grows because it is colder at its surface than at its base. In order to maintain local thermodynamic equilibrium throughout a growing layer, the salinity of the brine within the ice substructure increases toward the surface. However, because the density of salt water increases with salinity, buoyancy forces will tend to drive the fluid out of the matrix. If the crystals are sufficiently close to one another the permeability of the layer is decreased, and the matrix resists the buoyancy forces. As the layer grows the buoyancy forces and permeability evolve and ultimately the former win out and the brine trapped within the sea ice is abruptly released into the ocean below. Therefore the microstructure which controls the permeability has a direct influence on the evolution of the oceanographic mixed layer and hence ultimately the large-scale stability of the density structure of the Arctic Ocean. The principal features of this interplay are described using a mathematical model and are borne out by laboratory and field experiments (Wettlaufer et al., 1997, 2000).

Many important features of sea ice can be captured in laboratory experiments. The advantage of this approach is that one can close the mass balance, which is not possible in the field, and control the thermodynamic driving force, which is otherwise dictated by uncontrollable meteorological conditions. Ongoing challenges include developing appropriate measurement strategies. For example, how are local measurements of salinity of relevance to large-scale phenomena? New nondestructive methods (Notz et al., 2005) may ultimately be used for multiple autonomous deployments. The material is highly polycrystalline and hence within its interior the long time dynamics are influenced by impurities at the grain boundaries. On the surface, the redistribution of salt that defines the properties of "frost flowers," which control the radar properties of the ice (Nghiem et al., 1997), may be in part controlled by the interplay between grain boundaries and the dynamics of premelted films (Style and Worster, 2006). Finally, this liquid is home to cold adapted micro-organisms [recently reviewed by Mock and Thomas (2005)] and they not only tell us about polar ecosystems but also serve as a model for thinking about exobiology.

\section{Ice in the atmosphere}

Whenever we peer into the sky, day or night, the light that reaches our eyes will have been influenced by ice.
The temperature, weather, sun, wind-all are shaped by hydrometeors and their trajectories through the phases of water substance.

\section{Ozone chemistry on polar stratospheric cloud particles}

The discovery of the "Antarctic ozone hole" in 1985 (Farman, 1985), and subsequent discovery of thinning of the ozone layer in the Arctic (Barrie et al., 1988), raised world concern about the stability of the stratospheric ozone layer, which shields the biosphere from harmful ultraviolet radiation. It is now well established that polar stratospheric clouds (PSCs) play crucial roles in the destruction of the layer (Solomon, 1988; Abbatt, 1995; Molina et al., 1996; Carslaw and Peter, 1997; Ewing, 2006; Huthwelker et al., 2006). The cloud particles are the sites for halogen activation reactions, such as

$$
\begin{aligned}
& \mathrm{ClONO}_{3}+\mathrm{HCl} \rightarrow \mathrm{Cl}_{2}+\mathrm{HNO}_{3}, \\
& \mathrm{ClONO}_{2}+\mathrm{H}_{2} \mathrm{O} \rightarrow \mathrm{HOCl}+\mathrm{HNO}_{3}, \\
& \mathrm{HOCl}+\mathrm{HCl} \rightarrow \mathrm{Cl}_{2}+\mathrm{H}_{2} \mathrm{O} .
\end{aligned}
$$

These reactions convert the gases $\mathrm{ClONO}_{2}, \mathrm{HCl}$, and $\mathrm{HOCl}$ into the reactive form $\mathrm{Cl}_{2}$, which upon photolysis enters into ozone depleting catalytic cycles. The halogens primarily enter the atmosphere as components of chlorofluorocarbons and halons, from diverse applications such as refrigerants, solvents, firefighting agents, and chemicals for producing insulating foams. The gases may survive for decades in the atmosphere, so that even though the use of source chemicals has been severely restricted, ozone destruction will continue for many years.

The cloud particles are generally believed to be composed of various hydrates and concentrated solutions of sulfuric and nitric acids. The three thermodynamically stable solid phases found in PSC's; ice, nitric acid trihydrate or $\mathrm{NAT} \equiv \mathrm{HNO}_{3} \cdot 3 \mathrm{H}_{2} \mathrm{O}$, and sulfuric acid tetrahydrate or $\mathrm{SAT} \equiv \mathrm{H}_{2} \mathrm{SO}_{4} \cdot 4 \mathrm{H}_{2} \mathrm{O}$ have well-understood equilibria (Peter, 1999). However, depending on local thermodynamic conditions during cloud formation, and the presence of intermediate metastable states, the evolution of these particles can take substantially different paths. In one path a particle consists of a core of SAT and NAT surrounded by a shell of ice, whereas in the other path the core consists of ice and the shell can be either liquid or solid acid. Hence in one case further chemical uptake must occur on (doped) ice surfaces, whereas in the other the uptake may occur on a liquid. At issue is the nature of the particles' surfaces. For example, laboratory experiments have shown that under typical winter polar conditions the uptake of $\mathrm{HNO}_{3}$ on SAT substrates corresponds to approximately monolayer coverage, characteristic of reversible adsorption/ desorption processes (Zhang et al., 1996). However, uncertainties in the reactive uptake coefficients on NAT and SAT raise questions as to whether cloud particles involved are solid or liquid and make the latter more likely (Carslaw et al., 1997). A laboratory study of one of 
the reactions (the first of the three above) on two inert substrates indicated that the reaction mechanism appears to be determined by liquid water layers adsorbed on the surface (Molina et al., 1997). Such layers are able to remain unfrozen under polar conditions because of their high concentrations of the reactive chemicals; thus their liquid state is an active participant in the entire process. An outstanding question concerns whether impurity-induced surface melting can produce a sufficient amount of liquid so that, from the perspective of further chemisorption, path one is quantitatively similar to path two when acids are in the liquid phase. The question is much more than academic. Carslaw and Peter (1997) have analyzed recent experiments on the reaction $\mathrm{HCl}+\mathrm{ClONO}_{2} \rightarrow \mathrm{Cl}_{2}+\mathrm{HNO}_{3}$ and found a discrepancy in the reaction rates of up to two orders of magnitude. Therefore the most crucial mechanism driving stratospheric ozone destruction is not known to a sufficient accuracy to provide reliable input to global models that predict ozone losses and hence comparison with observations is presently out of reach. Such issues can be addressed by a careful study of the phase diagram of thin films of concentrated solutions; in essence, that of surface melting in a contaminated atmosphere, as described in Sec. II.

\section{Electrical charging in thunderstorms}

The study of thunderstorms has revealed considerable details of their electrical nature, but the principal mechanisms that electrify the clouds are still under study (MacGorman and Rust, 1998). It is possible that several mechanisms contribute to charging in particular thunderstorms; however, the strongest are those processes that involve ice and collisional charging, i.e., rebounding collisions between small ice particles and hail. The significant features of collisional charging have been obtained from laboratory studies. For example, (i) the systematic polarity is such that the particle that had been growing faster by vapor condensation becomes positively charged (Baker et al., 1987; Saunders, 1993, 1994); and (ii) mass exchange accompanies charging, and the transferred mass is fluid (Mason and Dash, 2000). Several microphysical mechanisms for collisional charging have been proposed, and are extensively reviewed by MacGorman and Rust (1998). In the following subsections we give a summary of proposed processes, and extensive review of those involving surface melting.

\section{a. Inductive charging}

One of the first charging mechanisms, originally proposed in the late 19th century, envisioned the polarization of a falling ice particle or water in the ambient field of a cloud (Elster and Geitel, 1888; Sartor, 1981; Mason, 1988). When such a particle impacts a cloud particle an amount of charge would be transferred from its bottom surface. However, Illingworth and Caranti (1985) estimated that the finite surface conductivity of particles limited the amount of charge that could be transferred during collisions. They concluded on this basis that the inductive mechanism could not account for the observed electrification of thunderstorms unless contaminants could have an unexpectedly large effect on the conductivity.

\section{b. Freezing potentials}

The discovery by Workman and Reynolds (1948) of electrical polarization during the freezing of dilute aqueous solutions, an effect caused by the differential rejection or incorporation of solute ions into the growing ice, led to the proposal that the mechanism could account for thunderstorm charging. Workman and Reynolds found electrical potentials as high as $230 \mathrm{~V}$ across the water-ice interface during the freezing process. Confirmation and elaboration of the results have been obtained in many experiments.

Gross (1965) and Bronshteyn and Chernov (1991) systematically studied the dependence of the effect on impurity species, concentration, and freezing rate. Takahashi (1969) pointed out that potential differences can be produced in the freezing of pure water, due to the difference of proton activation energy between liquid water and ice, and demonstrated that potentials as much as $100 \mathrm{~V}$ could be generated. The mechanism may participate in the charging of graupel and of melting snow. It is possible that this mechanism can be a significant contributor in some thunderstorms, although it does not produce the observed systematic polarity with respect to growth (Baker et al., 1987; Saunders, 1993, 1994).

\section{c. Thermoelectricity}

Latham and Mason (1961) observed that thermoelectricity can produce charging in thermal gradients associated with riming or vapor condensation. However, laboratory measurements of the effect demonstrate that it is too weak to be a major contributor to thunderstorm charging (Caranti et al., 1991; Avila and Caranti, 1994).

\section{d. Contact potentials}

Many investigators have ascribed collisional charging to contact potentials due to differences between the surface characters of particles (Buser and Aufdermaur, 1977; Gaskell and Illingworth, 1980; Caranti and Illingworth, 1983; Caranti et al., 1985; Keith and Saunders, 1990). Several experiments examined the dependence of charging on specific surface and environmental conditions: the relevance of surface damage, the importance of riming, and the sign of the transferred charge. These details have provided important clues for the development of microphysical models, which are reviewed in the following sections.

\section{e. Charging involving surface melting}

Turner and Stow (1984), citing evidence for premelted films on ice, proposed that collisional charging was mediated by such films. Their qualitative model assumed that the relevant differences in surface character were due to variations of local vapor pressure associated with growth. These differences would produce changes in sur- 
face ion density, sufficient for substantial charge exchange. The basic ion density was assumed to be a theoretical value estimated by Fletcher (1968) for the surface of a thin premelted ice film. However, this value corresponds to the density of molecular polarization, not free ion density; thus Fletcher's model does not actually allow any charge transfer. Regardless of the fallacy, Stow and Turner (1987) subsequently tested their idea with collisional charging experiments. Fine particles of pulverized hoar frost were dropped on an ice-covered target; charges on the projectiles were measured both before and after impacting the target. The results showed erratic charge transfer, which the authors attributed to wide variations in the local surface conditions. There was no evident dependence on the local pressure environment. A fraction of all incoming projectiles were uncharged initially, but charged after impact; for these "generative impacts" the target received a small positive charge. The results were at variance with previous studies, and it was concluded that the discrepancies were due to differences in water vapor saturation.

Baker and Dash $(1989,1994)$ developed a more detailed charging model based on premelted ice. They addressed the principal difficulty with the ion density in the work of Stow and Turner by considering dissociated water ions and ionic impurities. The concentrations of these ions would be modified in the neighborhood of the liquid-vapor interface, because of their tendency to screen electric fields due to the dipolar ordering of water molecules. Therefore there would be an excess concentration of free ions, with a polarity opposite to the molecular field, and these ions would be available for charge transfer. The charges would be carried in the liquid transferred between the melted layers, so that the charge transfer would be proportional to the net mass transfer. The mass transfer would be driven by differences of thickness and curvature, tending to drive liquid toward lower chemical potential. The model suffers from uncertainties in the strength and polarity of surface polarization, but it seemed that it might be consistent with laboratory observations of collisional charging. One of its most serious defects is that it cannot apply to temperatures appreciably colder than a few $\mathrm{K}$ below the melting point, i.e., within the rather restricted range of surface melting against pure water vapor.

\section{f. Collisional charging and surface disorder}

Mason and Dash (2000) undertook an experimental study motivated by the Baker-Dash model to test whether mass exchange accompanies charge transfer. They studied impacts between two ice-covered quartz crystals that were instrumented to record mass transfer associated with charge transfer in individual collisions. Impacts were studied as a function of mean environmental temperature, temperature difference, growth and evaporation rates, impact velocity, and time delay between events. The results showed that mass transfer accompanied charge transfer and was approximately proportional to it. The results also confirmed the observation from earlier collisional charging experi- ments that the faster growing ice particle becomes positively charged. A particularly interesting result was that statistical fluctuations of the transferred mass showed that it was fluid, even though the ambient temperature range extended well below the range of equilibrium surface melting. This finding provided an important clue for the development of a detailed model of the microphysical process.

Building on the observation of collisional melting (Mason and Dash, 2000) Dash et al. (2001) envisioned the charging process as a sequence of three stages; history before collision, contact, and separation. The microphysical basis of each stage is analyzed in quantitative detail.

Particle history before collision. The electrical nature of the ice particles stems from their growth history before collision. Well-ordered crystals can be grown from vapor if the growth rate is slow enough for the deposited molecules to diffuse on smooth surface facets to wellseparated attachment sites at steps, kinks, and ledges. However, if the deposition rate is too fast the molecules will nucleate into clusters on the surface which will thereby undergo kinetic roughening (see Sec. IV.E). The scale of roughness is set by a competition between the deposition rate and surface diffusion. The average time $\tau$ between arrivals of a vapor atom at a surface site is related to the site area $a$ and deposition rate $F$ by $\tau$ $=(a F)^{-1}$. If the surface diffusion coefficient is $D_{s}$, then during $\tau$ a freshly deposited molecule travels an average distance $\lambda=\sqrt{2 D_{s} \tau}$ (Einstein, 1956) before being buried by another molecule from the vapor. Therefore the average distance between steps on a facet is

$$
\lambda=\sqrt{2 D_{s} / a F} .
$$

In the case of ice, disordered surfaces contain ionic defects at corners and edges. The ionization leaves $\left(\mathrm{OH}^{-}\right)$ ions bound to the site by their remaining hydrogen bonds, while mobile positive ions diffuse deeper into ice, thus creating a charged double layer and a negative surface potential. The charged double layer, described by Eq. (23), in ice is similar to the phenomenon of an ionized surface in liquid water (Verwey and Overbeek, 1948; Israelachvili, 1992). The diffusion of ions into the bulk is driven by the entropy available in the larger number of sites in the interior, and is opposed by the electrostatic field between the two distributions. This competition produces a concentration profile characterized by a Debye length as defined by Eq. (24). The surface charge density $\sigma_{s}$ is proportional to the surface defect density. With Eq. (41), we have

$$
\sigma_{s}=\xi e a F / 2 D_{s},
$$

where $e$ is the elementary charge. The empirical parameter $\xi$ is the surface structural disorder factor, which can depend on growth conditions and history. Comparison with laboratory results yields the empirical value $\xi=4$ for the conditions of the Mason-Dash study.

During contact: Collisional melting. The impact between two ice particles causes temporary melting of a 
thin interfacial region. An estimate of the melt thickness $d_{m}$ is given by the simple expression

$$
d_{m}=\Delta E / A \rho_{\ell} q_{m} t,
$$

where $\Delta E$ is the inelastic energy deposit from the collision, $A$ is the area of contact, and the other quantities are as previously defined. As described in Sec. II.C, surface melting is enhanced by damage and other forms of disorder, which can cause melting at lower temperatures or thicker films than in the equilibrium case. The theory predicts that damage incurred in ice-ice collisions can cause melting of many molecular layers at temperatures well below $0{ }^{\circ} \mathrm{C}$. It provides a quantitative explanation for the measured mass transfer in ice-ice collisions (Mason and Dash, 2000). Furthermore, collisional melting between two ice particles destroys the site bonds of surface $\left(\mathrm{OH}^{-}\right)$ions, and allows them to diffuse in the liquid layer. For the relevant range of parameters, the confluence of the brief time of contact, about $0.1 \mathrm{~ms}$ (Mason and Dash, 2003), and the depth of the melt produced by the impact energy, is sufficient for complete mixing of $\mathrm{OH}^{-}$ions, but too short for most positive ions to escape from their deeper sites in the solid. The contact is also too brief to allow much refreezing of the collisionally melted liquid. Therefore collisional melting produces conditions that allow positive and negative ions to separate, and thus sets the stage for charge transfer between colliding particles.

Withdrawal. As collision partners separate they take roughly equal shares of the collisionally melted liquid. The precise division will depend on details such as local shape and surface roughness, so that sharing can only be estimated as a statistical average. Accordingly, in an average encounter particles share the liquid mass, together with its dissolved ions, so that the particle that had been growing more rapidly loses a greater number of its surface ions, and is thus charged positively. The mass transfer depends on the particles' initial temperature difference. In typical cases colliding particles have quite different histories; the more rapidly growing particle having been colder. Assuming equal sharing of the impact energy loss, the theory predicts that the colder particle suffers less collisional melting. Consequently, when the melted liquid is shared, the colder particle comes away with some of the warmer particle's mass.

Several significant results of laboratory measurements are explained by the theory. One of the strong clues that had helped the development of the theory was a conclusion drawn from earlier work, that the particle which had been undergoing more rapid vapor growth is charged positively (Baker et al., 1987; Saunders, 1993, 1994). Additional features seen in experiments by Mason and Dash (2000) showed that the charge transfer is proportional to the rate of growth at low rates, but increases more slowly and declines at increasingly high growth rate. These results are closely described by the theory as shown in Fig. 13. The agreement is obtained by fitting a single adjustable constant $\xi$ described in Eq. (42) representing the probability of ionization on growth steps. The theoretical curve shows the same trend as the

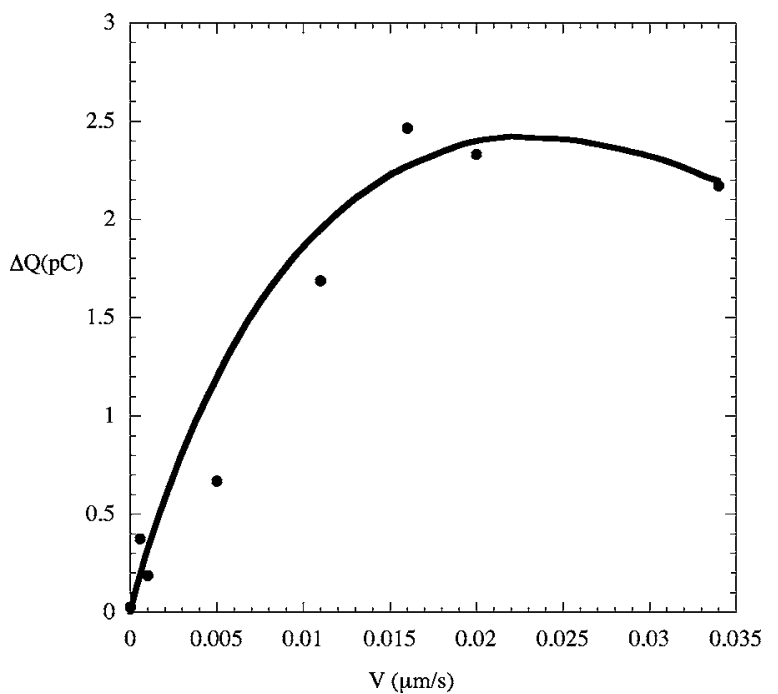

FIG. 13. Charge transfer versus growth rate; theory and experimental data are shown as a solid line and solid circles. The collisional area is $10^{-8} \mathrm{~m}^{2}$, the value of $\xi=4$, and the other experimental parameters as in Mason and Dash (2000). Adapted from Dash et al., 2001.

data over the entire range, including the falloff at high rates. The linear portion at low rates is explained by the proportionality of surface charge density on growth rate. At increasingly high growth rates the larger electric field between positive and negative charge distributions causes a contraction of the positive distribution toward the surface. Due to its shallower depth, during a collision a larger fraction of the positive charge is liberated into the melt layer, thereby neutralizing more negative charge.

\section{g. Charging involving particle shape}

Baker and Nelson (2002) proposed a collisional charging model with some features similar to the theory of Dash et al. (2001), and several in sharp contrast. The elements of the model include the following. The formation of a charged surface layer on each particle is due to electrostatic interactions among charged species just under the surface. The effective charge carriers are water ions and Bjerrum defects. The charges on Bjerrum defects are not free, but contribute to the internal field via polarization of the ice lattice, and ions move in the resulting field. The surface charge is calculated from conservation equations for charge carriers and their electrostatic interactions. Charge transfer depends on different precollision growth rates of particles, because of finite relaxation times of the ion densities. Melting occurs during collisions, because of the momentary high pressures and charges during contact. Charges are transferred within a small amount of liquid mass. The direction of mass transfer is controlled by different shapes of the contacting regions, driving material from sharper to flatter surfaces. The theory was applied to collisions between ice crystals and riming particles. The ice crystals are growing rapidly through vapor deposition, and they are assumed to have flat facets with sharp corners, while 
the riming particles have smoother surfaces. Typical collisions involve sharp corners of the ice crystals contacting flat regions of the riming particles. Dash and Wettlaufer (2003) criticized the Baker-Nelson model on several points. They disputed the assertion that the momentary high-contact pressures produce melting. Pressure melting requires that latent heat be supplied to the material, but in typical collisions the time is too brief to conduct enough heat to the contacting region. They also found that the argument for the direction of mass transfer was invalid; the normal pressure in a contact between particles with different radii of curvature is the same for both, and hence would produce no net mass transfer.

\section{h. Charging involving chemical impurities}

Jungwirth et al. (2005) have proposed a mechanism that is relevant to thunderstorms when it is clear that the atmosphere is contaminated. The authors note that the polarity of cloud-to-ground lightning tends to be negative under normal conditions, when sulfates dominate as cloud aerosols, and when smoke rich in chlorides and nitrates contaminates the atmosphere, the polarity tends to shift to positive strokes. Their proposed mechanism involves the differential incorporation of ions into freezing water, at lower average concentrations than in the normal Workman-Reynolds effect. They focus on the rejection of salts from a freezing cloud droplet, which can produce high concentrations of impurities in a surface layer. Ice crystals grow directly by deposition from the vapor, and it is assumed that they form an ordered ice surface, although the criteria for kinetic roughening (see Sec. IV.E) are not imposed. The graupel surface is formed from coalescing frozen cloud droplets, which originally nucleated on preexisting soluble aerosol particles (cloud condensation nuclei). The surface of such a frozen droplet is covered by a thin layer of a highly concentrated salt solution. The authors modeled the interfacial salt solution using molecular-dynamics simulation in a slab arrangement, but we note that they did not examine if the conditions for diffusive instabilities, as discussed in Sec. V.B, are operative. The principal result of the simulation is that aqueous ammonium cations and sulfate dianions have different interfacial concentrations; all sulfate dianions solvate deep in the aqueous bulk, but ammonium cations tend to be closer to the air interface. The distributions are apparently due to differences of size and polarizability, and are consistent with measurements of surface potentials. The authors modeled collisions between particles with such concentrations of impurities, and found substantial charging due to differences in the diffusional transport rates of the two ions. The charge polarity is within observations of the dependence of positive lightning on smoke contamination.

\section{i. Relationship to field studies}

Although vigorous debate continues to surround the causes of observed regional variations in lightning activ- ity some recent progress, using satellite data, helps to focus the search (Sherwood et al., 2006). Combining climatologies of lightning flash rate, for the period 19992003 from the Lightning Imaging Sensor and Optical Transient Detector on board the Tropical Rainfall Measuring Mission Satellite, with those of ice particle effective diameter $\left(D_{e}\right)$ obtained from the Advanced Very High Resolution Radiometer individual pixel radiance data at 3.7 and $11 \mu \mathrm{m}$, Sherwood, Phillips, and Wettlaufer (2006) showed that climatological maxima in lightning activity are associated with small $D_{e}$ of ice crystals near cumulonimbus cloud tops. Moreover, this correlation, unlike lightning's more well-known relationship with cloud top height, is consistent over land and ocean. Since multiple studies indicate that $D_{e}$ is reduced by atmospheric aerosol, this relationship strengthens previous suggestions of a role for aerosols as well as dynamics in electrification. Moreover, the angular distribution of backscattered radiance shows that modest $(\sim 10 \%) D_{e}$ decreases reflect large $(\sim 50 \%)$ increases in the number of small (less than $\simeq 30 \mu \mathrm{m}$ ) particles, a finding supported by cloud model simulations. Both relationships provide an important new test of cloud microphysics and/or electrification models and point to the importance of the role of small ice crystals, and hence relative growth rates in collisional charging. As described above, charging between unrimed crystals has recently been found experimentally (Mason and Dash, 2000) and explained (Dash et al., 2001), but laboratory experiments have long suggested that supercooled water is a critical, complicating factor in real storms (Takahashi, 1978). Therefore it is an important future research question to determine whether that indicates additional physics or the same physics operating in a more complicated environment.

\section{Extraterrestrial ice}

Water ice is one of the most common minerals in the universe. Motivated in part by the association between water and terrestrial life, the search for liquid water elsewhere within our solar system has received considerable attention. Many studies have focused on the case of Mars, where it has long been known that liquid water once flowed [for a discussion of some of the newest evidence, see Paige (2005)]. Recent findings point to an active role for aqueous liquids near the Martian surface within as short a time as the past few million years [see, e.g., Baker (2001, 2005)]. At present, the atmospheric pressure on Mars is much too low to permit liquid water to be a stable phase on the ground surface-even at low latitudes, where the temperature sometimes approaches $0{ }^{\circ} \mathrm{C}$. Nevertheless, stores of liquid water are thought to be located within the sediments. Indeed, it has been argued that conditions within the ice-bearing soils of an arid region of Antarctica known as the Dry Valleys might represent a valuable terrestrial analog for the Martian case (Dickinson and Rosen, 2003; Sletten et al., 2003). An interesting review of some of the potential 
consequences of water and ice coexisting in Martian sediments is given by Miller and Black (2003). Water ice is also present in the polar ice caps on Mars and premelted liquid can be expected to occur near the bases of these glaciers.

In our previous review (Dash et al., 1995) we noted the potentially central role of premelted liquid, from the vein/node/trijunction system to interfacially melted films, in biological viability. Since that time many corners of this rug have been upturned, from the potential habitat of deep ice core ice (Price, 2000; Campen et al., 2003; Mader et al., 2006) to the cold adapted organisms and biopolymer production in naturally occurring sea ice (Krembs et al., 2002; Junge et al., 2004; Mock and Thomas, 2005). Moreover, metabolic activity has been observed in fluid regions of ice at gigapascal pressures (Sharma et al., 2002). The implications are becoming increasingly compelling. Indeed, ice and evidence for liquid water have been identified in many other parts of the solar system, such as the moons of Jupiter, and ice is a major constituent of comets, the principal origins of which are the Kuiper belt and the Oort Cloud (Cruikshank, 2005). Moreover, the wide distribution of ice masses in the outer solar system provide essential clues for the structure of the solar nebula and initial phases of planet formation. One of the largest accumulations of extraterrestrial water ice yet discovered forms a crust of several kilometers thickness above a vast liquid ocean on Jupiter's satellite, Europa (e.g., Turtle and Pierazzo, 2001). It is interesting to speculate on the roles that premelted liquid and phase dynamics underlying sea ice evolution might play during the convection and resurfacing of this icy shell. We note that veins in terrestrial glacier ice have been shown to support active microbial communities (Campen et al., 2003), emphasizing the potential importance of these networks to astrobiology. Astronomers continue to find crystalline ice in some of the most surprising places, even beyond Neptune in the Kuiper belt, where only amorphous solid water had previously been thought to exist (Jewitt and Luu, 2004). A recent review of the physical state of ices (including the solid forms of volatile compounds other than water) associated with comets and icy satellites of our outer solar system is given by Roush (2001).

Ice is believed to have a role in the development of planetary systems. A crucial early step in their formation is the aggregation of small solid particles in gaseous protoplanetary disks such as the primordial solar nebula. Among small particles for which self-gravity is negligible, a sticking mechanism is necessary to hold the aggregate together even when relative velocities are small. Experiments show that ice grains develop bonds after short contact times, and bond strength increases sharply when temperature rises to $-5^{\circ} \mathrm{C}$ (Gubler, 1982). Further studies have found that several types of frost-covered surfaces stick together even at very low temperatures (Bridges et al., 1996). The authors speculate on the impact and surface conditions necessary to produce adhesion, but there is active debate regarding the dominant forces involved (Wang et al., 2005). It is suggested here that a possible mechanism is momentary collisional melting, as described in Secs. II.C and V.C.2.

Further discoveries will undoubtedly continue, producing new challenges and opportunities to divine the role of premelting on ice throughout the solar system and beyond.

\section{INTERFACIAL MELTING SANK AN AIRCRAFT CARRIER}

Few scientific articles open as dramatically as a report by Max Perutz (1948):

"In the autumn of 1942, when the German army had advanced almost to the gates of Alexandria and the U-boat war had reached one of its most critical phases, it was evident that some of the Allies' difficulties were due to their lack of air power in the distant battlefields."

Thus was born the idea to build an "unsinkable aircraft carrier" out of ice. The original memorandum, submitted by Geoffrey Pyke to the British Chief of Combined Operations, proposed that a natural or artificial iceberg be hollowed out to shelter aircraft and levelled to provide a takeoff and landing strip. It must have a mobility of at least a few knots to prevent drifting, and on board refrigeration to prevent melting. The construction would not take more than a minimum amount of strategic materials; most of the material would be free, and it could not be sunk. Winston Churchill ordered that the project be launched with the highest priority.

Preliminary design showed that the construction of such bergships would take enormous effort, and their size demanded more reliable data on the mechanical properties of ice than existed at that time. The minimum length of runway required for bombers at that time was $600 \mathrm{~m}$, and $60 \mathrm{~m}$ was considered the most desirable width. It must be self-propelled, with sufficient speed to prevent it drifting in the wind, and it must be difficult to sink. The final design specified a shape like a hollow square beam with tapered ends, a freeboard of $15 \mathrm{~m}$, minimum wall thickness $9 \mathrm{~m}$, and a displacement of $2.2 \times 10^{6}$ tons (six times heavier than the Queen Elizabeth). It would be cooled with compressed air circulating at $-30{ }^{\circ} \mathrm{C}$, generated by 16 refrigerant plants. The bergship would be propelled by over twenty 1100 BHP electric motors, needing turboelectric generators totalling 32000 BHP (Website).

Strength tests showed that ice was too brittle and variable to be dependable, but the outlook brightened when it was learned that the inclusion of a small amount of wood pulp might greatly improve the mechanical properties. Test results on a range of compositions yielded increasing strength up to a pulp content of $4 \%$, after which there was little increase. Tensile strength and modulus of rupture tests at -15 and $-17{ }^{\circ} \mathrm{C}$ were more than double those of pure ice. Bullet penetration depth was comparable to that of brickwork. In honor of the writer of the original memorandum, the composite was named "pykrete." However, creep tests showed that the 
material would deform plastically under moderate loads, with the creep rate rising rapidly as the melting point is approached. The composite would need to be maintained at temperatures no higher than $-15^{\circ} \mathrm{C}$, to prevent a ship from sagging under its own weight. In spite of these problems the project continued, and a 1:50 scale model was constructed on a lake in Alberta, Canada (Website). The engineers managed to keep the model frozen during the entire summer of 1943. But the cost of the project, especially the need to maintain the temperature well below the melting point, caused its abandonment. Perutz is quoted (Judson, 1979)

"Finally the Navy Department came to the conclusion that the amount of steel needed for reinforcement and to refrigerate all the ice needed to build one of these aircraft carriers, even in the winter of Newfoundland, would be greater than the amount needed to build the entire thing of steel."

"Nevertheless," Perutz (1948) writes, "the volume of first-rate data produced during a period of six months...under the pressure of war far exceeded the total volume of reliable work that had been done before on the mechanical properties of ice itself."

The article concluded with a discussion of creep tests on ice and pykrete. The results showed that creep continued even at $-25^{\circ} \mathrm{C}$, which disproved the then current idea that the plasticity of ice was due to pressure melting and regelation. It was suggested that the "quasiviscous flow" of the composite was probably similar to the mechanism of glacier flow and of polycrystalline materials in general. Today, we understand the creep of pykrete as combined effects of interfacial melting, the mobility of the melt, and recrystallization under stress. It is not clear if, with our present knowledge, we can now design a bergship with less demanding refrigeration; fortunately, there is no longer any need.

\section{SUMMARY}

In this review we have attempted to give a thorough treatment of the physics of ice premelting and a detailed development of the myriad of geophysical tendrils that are influenced by the basic phenomena. It has been some time since such a treatment was attempted from our particular perspective and a great deal of exciting work has been accomplished during that time, but doubtless, we have not covered all areas with equal thoroughness or acuity. In part, these are both cause and effect, for we have discovered that the field and its reach have been blossoming rapidly. We hope that our efforts here will provide a useful and enjoyable roadmap for future directions.

\section{ACKNOWLEDGMENTS}

We are grateful to Grae Worster for numerous important discussions and ongoing collaboration. We thank
Y. Furukawa, J. A. Neufeld, M. Spannuth, R. Style, E. S. Thomson, and L. A. Wilen for comments and feedback. This work was supported by the Bosack and Kruger Foundation, the U.S. National Science Foundation (Grant No. OPP0440841), the Department of Energy (Grant No. DE-FG02-05ER15741), University of Oregon, and Yale University. J.S.W. thanks the Institute of Theoretical Geophysics in the Department of Applied Mathematics \& Theoretical Physics, and Trinity College at the University of Cambridge where much of this paper was written.

\section{REFERENCES}

Aagaard, K., and E. Carmack, 1994, in The Polar Regions and Their Role in Shaping the Global Environment, edited by O. M. Johannessen, R. D. Muench, and J. E. Overland, Geophysical Monograph No. 85 (American Geophysical Union, Washington, D.C.), pp. 5-20.

Abbatt, J. P. D., 1995, J. Geophys. Res. 100, 14009.

Adam, J., 2005, Mon. Not. Am. Math. Soc. 52, 402.

Adams, E. E., D. A. Miller, and R. L. Brown, 2001, J. Appl. Phys. 90, 5782.

Alley, R. B., D. E. Lawson, E. B. Evenson, J. C. Strasser, and G. J. Larson, 1998, J. Glaciol. 44, 563.

Alley, R. B., D. E. Lawson, G. J. Larson, E. B. Evenson, and G. S. Baker, 2003, Nature (London) 424, 758.

Andersland, O. B., and B. Ladanyi, 2004, An Introduction to Frozen Ground Engineering (Chapman and Hall, New York), p. 363.

Asthana, R., and S. N. Tewari, 1993, J. Mater. Sci. 28, 5414.

Avila, E. E., and J. M. Caranti, 1994, J. Geophys. Res. 99, 10611.

Azouni, M. A., P. Casses, and B. Sergiani, 1997, Colloids Surf., A 122, 199.

Azouni, M. A., W. Kalita, and M. Yemmou, 1990, J. Cryst. Growth 99, 201.

Baker, B., M. B. Baker, E. R. Jayaratne, J. Latham, and C. P. R. Saunders, 1987, Q. J. R. Meteorol. Soc. 113, 1193.

Baker, I., and D. Cullen, 2003, J. Glaciol. 49, 184.

Baker, I., D. Cullen, and D. Iliescu, 2003, Can. J. Phys. 81, 1.

Baker, J. M., J. C. Dore, and P. Behrens, 1997, J. Phys. Chem. B 101, 6226.

Baker, M. B., and J. G. Dash, 1989, J. Cryst. Growth 97, 770. Baker, M. B., and J. G. Dash, 1994, J. Geophys. Res. 99, 10621. Baker, M. B., and J. Nelson, 2002, C. R. Phys. 3, 1293.

Baker, V. R., 2001, Nature (London) 412, 228.

Baker, V. R., 2005, Nature (London) 434, 280.

Balibar, S., H. Alles, and A. Ya. Parshin, 2005, Rev. Mod. Phys. 77, 317.

Balibar, S., F. Graner, and E. Rolley, 1993, Prog. Cryst. Growth Charact. Mater. 26, 5.

Bandyopadhyay, R., D. Liang, H. Yardimci, D. A. Sessoms, M. A. Borthwick, S. G. J. Mochrie, J. L. Harden, and R. L. Leheny, 2004, Phys. Rev. Lett. 93, 228302.

Barer, S. S., N. V. Churaev, B. V. Derjaguin, O. A. Kisileva, and V. D. Sobolev, 1980, J. Colloid Interface Sci. 74, 173.

Barer, S. S., V. I. Kvlividze, V. D. Sobolev, N. V. Churaev, and A. B. Kurzaev, 1977, Dokl. Akad. Nauk SSSR 235, 601.

Barnes, P. R. F., 2003, J. Appl. Phys. 93, 783.

Barnes, P. R. F., R. Mulvaney, K. Robinson, and E. W. Wolff, 2002, Ann. Glaciol. 35, 559. 
Barnes, P. R. F., E. W. Wolff, H. M. Mader, R. Udisti, E. Castellano, and R. Rothlisberger, 2003, J. Geophys. Res. 108, 4126.

Barnes, P. R. F., E. W. Wolff, D. C. Mallard, and H. M. Mader, 2003, Microsc. Res. Tech. 62, 62.

Barrie, L. A., J. W. Bottenheim, R. C. Schnell, P. J. Crutzen, and R. A. Rasmussen, 1988, Nature (London) 334, 138.

Bar-Ziv, R., and S. A. Safran, 1993, Langmuir 9, 2786.

Batchelor, G. K., 1973, An Introduction to Fluid Dynamics (Cambridge University Press, Cambridge, England), p. 615.

Beaglehole, D., 1991, J. Cryst. Growth 112, 663.

Beaglehole, D., and D. Nason, 1980, Surf. Sci. 96, 357.

Beaglehole, D., and D. Wilson, 1994, J. Phys. Chem. 98, 8096.

Beaudoin, G., P. Haljan, M. Paetkau, and J. R. Beamish, 1996, J. Low Temp. Phys. 105, 113.

Belissent-Funel, M.-C., 1993, J. Chem. Phys. 98, 4246.

Benatov, L., and J. S. Wettlaufer, 2004, Phys. Rev. E 70, 061606.

Beskow, G., 1935, in The Swedish Geological Society, C, no. 375, Year Book no. 3 (translated by J. O. Osterberg), Technological Institute, Northwestern University. Reprinted in Historical Perspectives in Frost Heave Research, edited by P. B. Black and M. J. Hardenberg, CRREL Special Report No. 91-23, p. 37.

Bindschadler, R. A., M. A. King, R. B. Alley, S. Anandakrishnan, and L. Padman, 2003, Science 301, 1087.

Bindschadler, R. A., P. L. Vornberger, M. A. King, and L. Padman, 2003, Ann. Glaciol. 36, 263.

Blachere, J. R., and J. E. Young, 1972, J. Am. Ceram. Soc. 55, 306.

Black, P. B., 1991, in Historical Perspectives in Frost Heave Research, edited by P. B. Black and M. J. Hardenberg, CRREL Special Report No. 91-23, p. 1.

Bluhm, H., T. Inoue, and M. Salmeron, 2000, Phys. Rev. B 61, 7760 .

Bluhm, H., and M. Salmeron, 1999, J. Chem. Phys. 111, 6947.

Bolling, G. F., and J. Cissé, 1971, J. Cryst. Growth 10, 56.

Borukhov, I., D. Andelman, and H. Orland, 1997, Phys. Rev. Lett. 79, 435.

Bottomley, J. T., 1872, Nature (London) 5, 185.

Bougamont, M., S. Tulaczyk, and I. Joughin, 2003, J. Geophys. Res. 108, 2223.

Boulton, G. S., E. M. Morris, A. A. Armstrong, and A. Thomas, 1979, J. Glaciol. 22, 3.

Bowden, F. P., 1953, Proc. R. Soc. London, Ser. A 217, 493.

Bowden, F. P., and T. P. Hughes, 1939, Proc. R. Soc. London, Ser. A 172, 280.

Boxe, C. S., A. J. Colussi, M. R. Hoffmann, D. Tan, J. Mastromarino, A. T. Case, S. T. Sandholm, and D. D. Davis, 2003, J. Phys. Chem. A 107, 11409.

Braun, J., A. Glebov, A. P. Graham, A. Menzel, and J. P. Toennies, 1998, Phys. Rev. Lett. 80, 2638.

Brewer, D. F., A. J. Dahm, W. S. Hutchins, and D. N. Williams, 1978, J. Phys. (Paris), Colloq. 39, C6-351.

Brewer, D. F., J. Rajendra, N. Sharma, and A. L. Thomson, 1990, Physica A 165, 569.

Bridges, F. G., D. S. Kimberley, D. N. C. Lin, R. Knight, and M. Zafra, 1996, Icarus 123, 422.

Bronshteyn, V. L., and A. A. Chernov, 1991, J. Cryst. Growth 112, 129.

Bronstein, V. L., Y. A. Itkin, and G. S. Ishkov, 1981, J. Cryst. Growth 52, 345.

Broughton, J. Q., A. Bonissent, and F. F. Abraham, 1981, J.
Chem. Phys. 74, 4029.

Broughton, J. Q., and G. H. Gilmer, 1983a, J. Chem. Phys. 79, 5095;

Broughton, J. Q., and G. H. Gilmer, 1983b, J. Chem. Phys. 79, 5105.

Broughton, J. Q., and G. H. Gilmer, 1983c, J. Chem. Phys. 79, 5114.

Broughton, J. Q., and G. H. Gilmer, 1984a, J. Chem. Phys. 84, 5741.

Broughton, J. Q., and G. H. Gilmer, 1984b, J. Chem. Phys. 84, 5759 .

Broughton, J. Q., and G. H. Gilmer, 1986, Phys. Rev. Lett. 56, 2692.

Brown, D. W., P. E. Sokol, and S. N. Ehrich, 1988, Phys. Rev. Lett. 81, 1019.

Bruschi, L., G. Torzo, and M. H. W. Chan, 1988, Europhys. Lett. 6, 541.

Buffat, P., and J. P. Borel, 1976, Phys. Rev. A 13, 2287.

Burak, Y., and D. Andelman, 2000, Phys. Rev. E 62, 5296.

Burak, Y., and D. Andelman, 2001, J. Chem. Phys. 114, 3271.

Bulau, J. R., H. S. Waff, and J. A. Tyburczy, 1979, J. Geophys. Res. 84, 6102.

Burton, W. K., N. Cabrera, and F. C. Frank, 1950, Philos. Trans. R. Soc. London, Ser. A 243, 299.

Buser, O., and A. N. Aufdermaur, 1977, in Electrical Processes in Atmospheres, edited by $\mathrm{H}$. Dolezalek and R. Reiter (Steinkopf, Darmstadt), pp. 294-301.

Cahn, J. W., 1960, Acta Metall. 8, 554.

Cahn, J. W., J. G. Dash, and H.-Y. Fu, 1992, J. Cryst. Growth 123, 101.

Cahn, J. W., W. B. Hillig, and G. W. Sears, 1964, Acta Metall. 12, 1421.

Campen, R. K., T. Sowers, and R. B. Alley, 2003, Geology 31, 231.

Caranti, J. M., E. E. Avila, and M. A. Re, 1991, J. Geophys. Res. 96, 15365.

Caranti, J. M., and A. J. Illingworth, 1983, J. Phys. Chem. 87, 4125.

Caranti, J. M., A. J. Illingworth, and S. J. Marsh, 1985, J. Geophys. Res. 90, 6041.

Carsey, F., A. Behar, A. L. Lane, V. Realmuto, and H. Engelhardt, 2002, J. Glaciol. 48, 622.

Carslaw, K. S., and T. Peter, 1997, Geophys. Res. Lett. 24, 1743.

Carslaw, K. S., T. Peter, and R. Muller, 1997, Geophys. Res. Lett. 24, 1747.

Chamberlain, E. J., and A. J. Gow, 1979, Eng. Geol. (Amsterdam) 13, 73.

Chen, J.-P., and P. J. Crutzen, 1994, J. Geophys. Res. 99, 18847. Chernov, A. A., and A. M. Mel'nikova, 1966, Sov. Phys. Crystallogr. 10, 672 .

Chernov, A. A., and L. V. Mikheev, 1989, Physica A 157, 1042. Chernov, A. A., and D. E. Temkin, 1977, in Current Topics in Materials Sci., Vol. 2, 1976, Crystal Growth and Materials, edited by E. Kaldis and H. J. Scheel (North-Holland, Amsterdam), p. 3.

Chernov, A. A., D. E. Temkin, and A. M. Mel'nikova, 1976, Sov. Phys. Crystallogr. 21, 369.

Cho, H., P. B. Shepson, L. A. Barrie, J. P. Cowin, and R. Zaveri, 2002, J. Phys. Chem. B 106, 11226.

Christoffersen, P., and S. Tulaczyk, 2003, J. Geophys. Res. 108, 2222.

Clapsaddle, C., and D. Lamb, 1989, Geophys. Res. Lett. 16, 
1173.

Cohen, D., N. R. Iverson, T. S. Hooyer, U. H. Fischer, M. Jackson, and P. L. Moore, 2005, J. Geophys. Res. 110, F02007. Colbeck, S., 1988, J. Glaciol. 34, 78.

Colbeck, S., 1991, J. Glaciol. 37, 228.

Colbeck, S., 1992, Cold Regions Research Laboratory Special Report No. 92.

Colbeck, S., 1993, Cold Regions Research Laboratory Special Report No. 93.

Colbeck, S., and G. C. Warren, 1991, J. Glaciol. 37, 228.

Colbeck, S. C., 1979, Philos. Mag. A 39, 13.

Colbeck, S. C., 1982, Rev. Geophys. Space Phys. 20, 45.

Colbeck, S. C., 1998, J. Appl. Phys. 84, 4585.

Conklin, M. H., and R. C. Bales, 1993, J. Geophys. Res. 98, 16851.

Conklin, M. H., R. A. Sommerfeld, S. K. Laird, and J. E. Villinski, 1993, Atmos. Environ., Part A 27A, 159.

Conway, H., G. Catania, C. F. Raymond, A. M. Gades, T. A. Scambos, and H. Engelhardt, 2002, Nature (London) 419, 465.

Conway, H., and C. F. Raymond, 1993, J. Glaciol. 39, 635.

Coombes, C. J., 1972, J. Phys. F: Met. Phys. 2, 441.

Corte, A. E., 1962, J. Geophys. Res. 67, 1085.

Corte, A. E., 1963, Science 142, 499.

Corte, A. E., 1969, Rev. Eng. Geol. 2, 119.

Cruikshank, D. P., 2005, Space Sci. Rev. 116, 421.

Cuffey, K. M., H. Conway, A. Gades, B. Hallet, C. F. Raymond, and S. Whitlow, 2000, J. Geophys. Res. 105, 27895.

Cuffey, K. M., H. Conway, B. Hallet, A. M. Gades, and C. F. Raymond, 1999, Geophys. Res. Lett. 26, 751.

Cullen, D., and I. Baker, 2001, Microsc. Res. Tech. 55, 198.

Curtin, W. A., and N. W. Ashcroft, 1985, Phys. Rev. A 32, 2909.

Czihak, C., 2000, Physica B 276, 286.

Dal Corso, A., and E. Tosatti, 1993, Phys. Rev. B 47, 9742.

Daniels, J., 1971, Opt. Commun. 3, 240.

Dansgaard, W., S. J. Johnsen, H. B. Clausen, D. Dahl-Jensen, N. S. Gundestrup, C. U. Hammer, C. S. Hvidberg, J. P. Steffensen, A. E. Sveinbjornsdottir, J. Jouzel, and G. Bond, 1993, Nature (London) 364, 218.

Dash, J. G., 1988, in Proceedings of the Solvay Conference on Surface Science, edited by F. W. deWette (Springer-Verlag, Berlin), p. 142.

Dash, J. G., 1989a, Contemp. Phys. 30, 89.

Dash, J. G., 1989b, Science 246, 1591.

Dash, J. G., 2002, Contemp. Phys. 43, 427.

Dash, J. G., 2003, Scr. Mater. 49, 1003.

Dash, J. G., H.-Y. Fu, and J. S. Wettlaufer, 1995, Rep. Prog. Phys. 58, 115.

Dash, J. G., V. A. Hodgkin, and J. S. Wettlaufer, 1999, J. Stat. Phys. 95, 1311.

Dash, J. G., B. L. Mason, and J. S. Wettlaufer, 2001, J. Geophys. Res. 106, 20395.

Dash, J. G., and J. S. Wettlaufer, 2003, C. R. Phys. 4, 721.

Davidson, G. P., and J. F. Nye, 1985, Cold Regions Sci. Technol. 11, 141.

de Gennes, P. J. G., 1985, Rev. Mod. Phys. 57, 827.

De Koning, J., G. De Groot, and G. J. van Ingen Schenau, 1992, J. Biomech. 25, 573.

de la Chapelle, S., O. Castelnau, V. Lipenkov, and P. Duval, 1998, J. Geophys. Res. 103, 5091.

Derjaguin, B. V., and B. V. Churaev, 1980, Kolloidn. Zh. 42, 842.

Derjaguin, B. V., and L. Landau, 1941, Acta Physicochim.
URSS 14, 633.

Dickinson, W. W., and M. R. Rosen, 2003, Geology 31, 199.

Dietrich, S., 1988, in Phase Transitions and Critical Phenomena, edited by C. Domb and J. Lebowitz (Academic, New York), Vol. 12.

Domine, F., L. Chaix, and L. Hanot, 2000, J. Colloid Interface Sci. 227, 104.

Doppenschmitt, A. H., and H. J. Butt, 2000, Langmuir 16, 6709.

Dosch, H., A. Lied, and J. Bilgram, 1995, Surf. Sci. 327, 145.

Dosch, H., A. Lied, and J. Bilgrim, 1996, Surf. Sci. 366, 43.

Duffy, J. A., N. J. Wilkinson, H. M. Fretwell, M. A. Alam, and

R. Evans, 1995, J. Phys.: Condens. Matter 7, L713.

Durham, W. B., S. H. Kirby, and L. A. Stern, 1997, J. Geophys. Res. 102, 16293.

Durham, W. B., S. H. Kirby, and L. A. Stern, 2001, J. Geophys. Res. 106, 11031.

Duval, P., M. F. Ashby, and I. Anderman, 1983, J. Phys. Chem. 87, 4066.

Duval, P., and M. Montagnat, 2002, J. Geophys. Res. 107, 2082.

Dzyaloshinskii, I. E., E. M. Lifshitz, and L. P. Pitaevskii, 1961, Adv. Phys. 10, 165.

Eastman, T., and D.-M. Zhu, 1995, J. Colloid Interface Sci. 172, 297.

Edwards, S. A., and D. R. M. Williams, 2004, Phys. Rev. Lett. 92, 248303.

Einstein, A., 1956, Investigations on the Theory of the Brownian Movement, edited with notes by R. Fürth, translated by A. D. Cowper (Dover, New York), pp. 1-119.

Elbaum, M., 1991a, Phys. Rev. Lett. 67, 2982.

Elbaum, M., S. G. Lipson, and J. G. Dash, 1993, J. Cryst. Growth 129, 491.

Elbaum, M., and M. Schick, 1991, Phys. Rev. Lett. 66, 1713.

Elbaum, M., and J. S. Wettlaufer, 1993, Phys. Rev. E 48, 3180.

Elbaum, M. E., 1991b, Ph.D thesis (University of Washington, Seattle).

Elster, J., and H. Geitel, 1888, Meteorol. Z. 5, 95.

Engemann, S., H. Reichert, H. Dosch, J. Bilgram, V. Honkimaki, and A. Snigirev, 2004, Phys. Rev. Lett. 92, 205701.

EPICA Community Members, 2004, Nature (London) 429, 623.

Evans, D. C. B., J. F. Nye, and K. J. Cheeseman, 1976, Proc. R. Soc. London, Ser. A 347, 493.

Everett, D. H., 1961, Trans. Faraday Soc. 57, 1541.

Ewing, G. E., 2004, J. Phys. Chem. B 108, 15953.

Ewing, G. E., 2006, Chem. Rev. 106, 1511.

Faraday, M., 1850, The Athenaeum 1181, 640.

Faraday, M., 1933, Faraday's Diary (Bell and Sons, London), Vol. IV, p. 79.

Farman, J. C., J. B. Gardiner, and J. D. Shanklin, 1985, Nature (London) 315, 207.

Fenzl, W., 2003, Europhys. Lett. 64, 64.

Fisher, D. A., 1987, International Association of Hydrological Sciences Publication 170 (Symposium at Vancouver 1987The Physical Basis of Ice Sheet Modelling), p. 45.

Fletcher, N. H., 1962, Philos. Mag. 7, 255.

Fletcher, N. H., 1968, Philos. Mag. 18, 1287.

Forland, K. S., T. Forland, and S. K. Ratke, 1988, Irreversible Thermodynamics (Wiley, New York), p. 235.

Foster, J. L., C. Sun, J. P. Walker, R. Kelly, A. Chang, J. Dong, and H. Powell, 2005, Remote Sens. Environ. 94, 187.

Fountain, A. G., R. W. Jacobel, R. Schlichting, and P. Jansson, 2005, Nature (London) 433, 618. 
Fowler, A. C., 1984, Geophys. Astrophys. Fluid Dyn. 28, 99.

Fowler, A. C., 1989, SIAM J. Appl. Math. 49, 991.

Fowler, A. C., and W. B. Krantz, 1994, SIAM J. Appl. Math. 54, 1650.

Fowler, A. C., and C. G. Noon, 1993, Cold Regions Sci. Technol. 21, 327.

Frank, F. C., 1968, Nature (London) 220, 350.

Frank, F. C., 1974, J. Glaciol. 13, 535.

French, R. W., 2000, J. Am. Ceram. Soc. 83, 211.

Frenkel, J., 1946, Kinetic Theory of Liquids (Clarendon, Oxford).

Frenken, J. W. M., and J. F. van der Veen, 1985, Phys. Rev. Lett. 54, 134.

Fu, H.-Y., and J. G. Dash, 1993, J. Colloid Interface Sci. 159, 343.

Fujita, S., N. Axuma, H. Motoyama, T. Kameda, H. Narita, S. Matoba, M. Igarashi, M. Kohno, Y. Fuji, and O. Watanabe, 2002, Ann. Glaciol. 35, 321.

Fukazawa, H., K. Sugiyama, S. J. Mae, H. Narita, and T. Hondoh, 1998, Geophys. Res. Lett. 25, 2845.

Furukawa, Y., 1997, Chem. Unserer Zeit 31, 58.

Furukawa, Y., and I. Ishikawa, 1993, J. Cryst. Growth 128, 1137.

Furukawa, Y., and S. Kohata, 1993, J. Cryst. Growth 129, 571. Furukawa, Y., and H. Nada, 1997, in Advances in Understanding Crystal Growth Mechanisms, edited by T. Nishinaga et al. (Elsevier B. V., North-Holland, Amsterdam), p. 559.

Furukawa, Y., M. Yamamoto, and T. Kuroda, 1987, J. Cryst. Growth 82, 665.

Gaskell, W., and A. J. Illingworth, 1980, Q. J. R. Meteorol. Soc. 106, 841.

Gay, J. M., J. Suzanne, J. G. Dash, and H.-Y. Fu, 1992, J. Cryst. Growth 125, 33.

Giddings, J. C., and E. LaChapelle, 1962, J. Geophys. Res. 67, 2377.

Gilpin, R. R., 1979, J. Colloid Interface Sci. 68, 235.

Gilpin, R. R., 1980a, J. Colloid Interface Sci. 77, 435.

Gilpin, R. R., 1980b, Water Resour. Res. 16, 918.

Gilpin, R. R., 1980c, J. Colloid Interface Sci. 74, 44.

Gladkich, N. T., R. Niedermayer, and K. Spiegel, 1966, Phys. Status Solidi 15, 181.

Glen, J. W., 1955, Proc. R. Soc. London, Ser. A 228, 519.

Goldsby, D. L., 2006, in Glaciology and the Earth's Changing Environment, edited by P. Knight (Blackwell, Oxford, in press), Chap. 60.

Goldsby, D. L., and D. L. Kohlstedt, 1997, Scr. Mater. 37, 1399.

Goldsby, D. L., and D. L. Kohlstedt, 2001, J. Geophys. Res. 106, 11017.

Goldsby, D. L., and D. L. Kohlstedt, 2002, J. Geophys. Res. 107, 2313.

Golecki, I., and C. Jaccard, 1978, J. Phys. C 11, 4229.

Gross, G. W., 1965, J. Geophys. Res. 70, 2291.

Gubler, H., 1982, J. Glaciol. 28, 457.

Hales, T. C., and J. R. Roering, 2005, Geology 33, 701.

Hallet, B., 1981, Ann. Glaciol. 2, 23.

Hallet, B., 1990, Can. J. Phys. 68, 842.

Hallet, B., J. S. Walder, and C. W. Stubbs, 1991, Permafrost Periglacial Process. 2, 283.

Harrison, W. D., 1972, J. Glaciol. 11, 15.

Haymet, A. D. J., and D. W. Oxtoby, 1974, J. Chem. Phys. 74, 2559.

Henry, K. S., 2000, in Cold Regions Research and Engineering Laboratory Technical Report No. ERDC/CRREL TR-00-16.
Herring, C., 1951a, Phys. Rev. 82, 87.

Herring, C., 1951b, in The Physics of Powder Metallurgy, edited by W. E. Kingston (McGraw-Hill, New York), p. 143.

Herron, M. M., and C. C. Langway, 1980, J. Glaciol. 25, 373.

Heyraud, J. C., and J. J. Mètois, 1986, Surf. Sci. 177, 213.

Heyraud, J. C., and J. J. Mètois, 1987, J. Cryst. Growth 82, 269. Hills, B. P., 1994, Mol. Phys. 82, 751.

Hiroi, M., T. Mizusaki, T. Tsuneto, A. Hirai, and K. Eguchi, 1989, Phys. Rev. B 40, 6581.

Hobbs, P. V., 1974, Ice Physics (Clarendon, Oxford), p. 837.

Hoekstra, P., and A. Delaney, 1974, J. Geophys. Res. 79, 1699.

Hoekstra, P., and R. D. Miller, 1967, J. Colloid Interface Sci. 25, 166.

Hosler, C. L., D. C. Jensen, and L. Goldshlak, 1957, J. Meteorol. 14, 415 .

Hsieh, T. E., and R. W. Balluffi, 1989, Acta Metall. 37, 1637.

Hutchinson, J. W., 1977, Metall. Trans. A 8A, 1465.

Huthwelker, T., M. Ammann, and T. Peter, 2006, Chem. Rev. 106, 1375 .

Huthwelker, T., D. Lamb, M. Baker, B. Swanson, and T. Peter, 2001, J. Colloid Interface Sci. 238, 147.

Huthwelker, T., M. E. Malmstrom, F. Helleis, G. K. Moortgat, and T. Peter, 2004, J. Phys. Chem. 108, 6302.

Ikeda-Fukazawa, T., and K. Kawamura, 2004, J. Chem. Phys. 120, 1395.

Illingworth, A. J., and J. M. Caranti, 1985, J. Geophys. Res. 95, 6033.

Inglis, D. R., and A. E. Corte, 1965, Science 148, 1616.

Ishizaki, T., M. Maruyama, Y. Furukawa, and J. G. Dash, 1996, J. Cryst. Growth 163, 455.

Israelachvili, J. N., 1992, Intermolecular and Surface Forces (Academic, New York).

Iverson, N. R., 1993, Geology 21, 559.

Iverson, N. R., 2000, Earth Surf. Processes Landforms 25, 881.

Iverson, N. R., D. Cohen, T. S. Hooyer, U. H. Fischer, M. Jackson, P. L. Moore, G. Lappegard, and J. Kohler, 2003, Science 301, 81.

Iverson, N. R., and D. J. Semmens, 1995, J. Geophys. Res. 100, 10219.

Jackson, C. L., and G. B. McKenna, 1990, J. Chem. Phys. 93, 9002.

Jackson, K. A., 1958, in Growth and Perfection of Crystals, edited by R. H. Doremus, B. W. Roberts, and D. Turnbull (Wiley, New York), p. 319.

Jellinek, H. H. G., 1967, J. Colloid Interface Sci. 25, 192.

Jewitt, D. C., and J. Luu, 2004, Nature (London) 432, 731.

Jiang, Q., 2001, J. Phys.: Condens. Matter 13, L397.

Johnsen, S., 2005, private communication.

Johnsen, S. J., H. B. Clausen, K. M. Cuffey, G. Hoffmann, J. Schwander, and T. Creyts, 2000, in Physics of Ice Core Records, edited by T. Hondoh (Hokkaido University Press, Sapporo), p. 121.

Johnsen, S. J., H. B. Clausen, J. Jouzel, J. Schwander, Á. E. Sveinbjörnsdóttir, and J. White, 1999, in Ice Physics and the Natural Environment, edited by J. S. Wettlaufer, J. G. Dash, and N. Untersteiner, NATO Advanced Studies Institute, No. 56, Series I: Global Environmental Change (Springer-Verlag, Berlin), p. 89.

Johnston, G. H., Ed., 1981, Permafrost Engineering Design and Construction (Wiley, Toronto), p. 540.

Joly, J., 1887, Proc. R. Soc. Dublin 5, 453.

Joughin, I., W. Abdalati, and M. Fahnestock, 2004, Nature (London) 432, 608. 
Journal of Glaciology (Int. Glaciological Soc., Cambridge, England).

Judson, H. F., 1979, The Eighth Day of Creation (Simon and Schuster, New York), p. 544.

Junge, K., H. Eicken, and J. W. Deming, 2004, Appl. Environ. Microbiol. 70, 550.

Jungwirth, P., D. Rosenfeld, and V. Buch, 2005, Atmos. Res. 76, 190.

Kamb, B., 1970, Rev. Geophys. Space Phys. 8, 683.

Kamb, B., 1991, J. Geophys. Res. 96, 16585.

Karim, O. A., and A. D. J. Haymet, 1987, J. Chem. Phys. 89, 6889.

Keith, W. D., and C. P. R. Saunders, 1990, Atmos. Res. 25, 445.

Kessler, M. A., and B. T. Werner, 2003, Science 299, 380.

Ketcham, W. M., and P. V. Hobbs, 1969, Philos. Mag. 19, 1161.

Khvorostyanov, V. I., and J. A. Curry, 2004, J. Phys. Chem. A 108, 11073.

Kikuchi, R., and J. W. Cahn, 1980, Phys. Rev. B 21, 1893.

Knight, C. A., 1971, Philos. Mag. 23, 153.

Kondo, Y., Y. Kodama, Y. Hirayoshi, T. Misuzaki, A. Hirai, and K. Eguchi, 1987, J. Appl. Phys. 26, 303.

Konrad, J.-M., and C. Duquennoi, 1993, Water Resour. Res. 29, 3109.

Konrad, J. M., and N. R. Morgenstern, 1981, Can. Geotech. J. 18, 428.

Koo, K., R. Anath, and W. N. Gill, 1991, Phys. Rev. A 44, 3782.

Kouchi, A., Y. Furukawa, and T. Kuroda, 1987, J. Phys. (Paris), Colloq. 48, C1-675.

Krembs, C., K. Junge, H. Eicken, and J. W. Deming, 2002 , Deep-Sea Res., Part I 49, 2163.

Kristensen, J. K., and R. M. Cotterill, 1977, Philos. Mag. 36, 437.

Kroes, G.-J., 1992, Surf. Sci. 275, 365.

Kuroda, T., and R. Lacmann, 1982, J. Cryst. Growth 56, 189.

Kvlividze, V. I., V. F. Kisilev, A. B. Kurzaev, and L. A. Ushakova, 1974, Surf. Sci. 44, 60.

Landais, A., J. P. Steffensen, N. Caillon, J. Jouzel, V. MassonDelmotte, and J. Schwander, 2004, J. Geophys. Res. 109, D06103.

Latham, J., and B. J. Mason, 1961, Proc. R. Soc. London, Ser. A 260, 523 .

Leshansky, A. M., and J. F. Brady, 2005, J. Fluid Mech. 527, 141.

Li, J.-C., 1993, Phys. Rev. B 48, 6716.

Liang, H., J. M. Martin, and T. L. Mogne, 2003, Acta Mater. 51, 2639.

Liang, H., J. M. Martin, and T. L. Mogne, 2005, J. Appl. Phys. 97, 043525.

Libbrecht, K. G., 2005, Rep. Prog. Phys. 68, 855.

Liezhao, C., D. F. Brewer, C. Girit, E. N. Smith, and J. D. Reppy, 1986, Phys. Rev. B 33, 106.

Lipowsky, R., 1986, Phys. Rev. Lett. 57, 2876.

Lipowsky, R., and W. Speth, 1983, Phys. Rev. B 28, 3983.

Lipp, G., and C. Körber, 1993, J. Cryst. Growth 130, 475.

Lipson, S. G., and E. Polturak, 1987, Prog. Low Temp. Phys. 11, 128.

Lliboutry, L., 1971, J. Glaciol. 10, 15.

Lobkovsky, A. E., and J. A. Warren, 2002, Physica D 164, 202.

Løwen, H., 1994, Phys. Rep. 237, 249.

Luo, J., and Y.-M. Chiang, 2000, Acta Mater. 48, 4501.

MacGorman, D. R., and W. D. Rust, 1998, The Electrical Nature of Storms (Oxford University Press, New York).

MacMillan, D., 1925, Four Years in the White North (The
Medici Society of America, Boston/New York).

Mader, H. M., 1992a, J. Glaciol. 38, 359.

Mader, H. M., 1992b, J. Glaciol. 38, 333.

Mader, H. M., M. E. Pettitt, J. L. Wadham, E. W. Wolff, and R. J. Parkes, 2006, Geology 34, 169.

Mahanty, J., and B. W. Ninham, 1976, Dispersion Forces (Academic, London).

Marshall, H. P., H. Conway, and L. A. Rasmussen, 1999, Cold Regions Sci. Technol. 30, 35.

Marshall, H. P., J. T. Harper, W. T. Pfeffer, and N. F. Humphrey, 2002, Geophys. Res. Lett. 29, 2146.

Maruyama, M., 1988, J. Cryst. Growth 89, 415.

Maruyama, M., J. G. Dash, and H.-Y. Fu, 1992, J. Cryst. Growth 123, 101.

Maruyama, M., Y. Kishimoto, and T. Sawada, 1997, J. Cryst. Growth 172, 521.

Maruyama, M., T. Satoi, S. Taniguchi, M. Kawamura, S. Kodera, Y. Kishimoto, and Y. Furukawa, 2000, Jpn. J. Appl. Phys., Part 1 39, 6696.

Mason, B. J., 1988, Proc. R. Soc. London, Ser. A 327, 303.

Mason, B. L., and J. G. Dash, 2000, J. Geophys. Res. 105, 10185.

Matsuoka, N., 2001, Earth Surf. Processes Landforms 26, 601. Miller, R. D., 1978, Proceedings of the Third International Conference on Permafrost (NRC Canada, Ottawa), p. 707.

Miller, R. D., and P. B. Black, 2003, J. Geophys. Res. 108, 8041.

Mitra, S. K., S. Barth, and H. R. Pruppacher, 1990, Atmos.

Environ., Part A 24A, 2307.

Mizuno, Y., and N. Hanafusa, 1987, J. Phys. (Paris), Colloq. 48, C1-511.

Mizusaki, T., and M. Hiroi, 1995, Physica B 210, 403.

Mock, T., and D. N. Thomas, 2005, Environ. Microbiol. 7, 605. Molina, M., L. T. Molina, and C. E. Kolb, 1996, Annu. Rev. Phys. Chem. 47, 327.

Molina, M., L. T. Molina, R. Zheng, R. F. Meads, and D. D. Spencer, 1997, Geophys. Res. Lett. 24, 1619.

Mori, A., M. Maruyama, and Y. Furukawa, 1996, J. Phys. Soc. Jpn. 65, 4001.

Morishige, K., 1997, J. Chem. Phys. 107, 6965.

Morishige, K., 2005, J. Chem. Phys. 122, 44711.

Mulvaney, R., E. W. Wolff, and K. Oates, 1988, Nature (London) 331, 247.

Mutou, Y., K. Watanabe, T. Ishizaki, and M. Mizoguchi, 1998, in Proceedings of the Seventh International Conference on Permafrost, 1998, Yellowknife, Canada, edited by A. G. Lewkowicz and M. Allard (Université Laval, Montreal), p. 283.

Nada, H., and Y. Furukawa, 1997, Appl. Surf. Sci. 121, 445.

Nagashima, K., and Y. Furukawa, 1997, J. Phys. Chem. B 101, 6174.

Nakano, Y., 1990, Cold Regions Sci. Technol. 17, 207.

Nakaya, U., 1954, Snow Crystals (Harvard University Press, New York).

Nansen, F., 1994, Ski Over Greenland (Aventura, Oslo).

Nenow, D., and A. Trayanov, 1989, Surf. Sci. 213, 488.

Netz, R. R., 2001a, Eur. Phys. J. E 5, 557.

Netz, R. R., 2001b, Eur. Phys. J. E 5, 189.

Netz, R. R., and D. Andelman, 1997, Phys. Rev. E 55, 687.

Ng, F., and H. Conway, 2004, Geology 32, 481.

Nghiem, S. V., S. Martin, D. K. Perovich, R. Kwok, R. Drucker, and A. J. Gow, 1997, J. Geophys. Res. 102, 3357.

Notz, D., J. S. Wettlaufer, and M. G. Worster, 2005, J. Glaciol. 51, 159. 
Nunn, K. R., and D. N. Rowell, 1967, Philos. Mag. 16, 1281. Nye, J. F., 1969, Proc. R. Soc. London, Ser. A 311, 445.

Nye, J. F., 1970, Proc. R. Soc. London, Ser. A 315, 381.

Nye, J. F., 1989, J. Glaciol. 35, 17.

Nye, J. F., 1991a, J. Glaciol. 37, 401.

Nye, J. F., 1991b, J. Cryst. Growth 113, 465.

Nye, J. F., 1992, in Physics and Chemistry of Ice, edited by N. Meano and T. Hondo (Hokkaido University Press, Sapporo), p. 200.

Nye, J. F., 1998, J. Glaciol. 44, 467.

Nye, J. F., and F. C. Frank, 1973, in International Assoc. of Sci. Hydrology Pub. 95 (Symposium at Cambridge 1969Hydrology of Glaciers), p. 157.

Nye, J. F., and S. Mae, 1972, J. Glaciol. 11, 81.

Ocampo, J., and J. Klinger, 1983, J. Phys. Chem. 87, 4167.

Ohno, H., M. Igarashi, and T. Hondoh, 2005, Earth Planet. Sci. Lett. 232, 171.

Omenyi, S. N., and A. W. Neumann, 1976, J. Appl. Phys. 47, 3956.

O’Neill, K., 1983, Cold Regions Sci. Technol. 6, 275.

O’Neill, K., and R. D. Miller, 1985, Water Resour. Res. 21, 281.

Ostroumov, V., R. Hoover, N. Ostroumov, and B. V. Vliet-

Lanoë, 2001, Cold Regions Sci. Technol. 32, 175.

Oxtoby, D. W., 1999, in Ice Physics and the Natural Environment, edited by J. S. Wettlaufer, J. G. Dash, and N. Untersteiner, NATO Advanced Studies Institute No. 56, Series I: Global Environmental Change (Springer-Verlag, Heidelberg), p. 39.

Paige, D. A., 2005, Science 307, 1575.

Pandit, R., M. Schick, and M. Wortis, 1982, Phys. Rev. B 26, 5112.

Parsegian, V. A., 1975, in Physical Chemistry: Enriching Topics from Colloid and Surface Science, edited by H. van Olphen and K. J. Mysels (Theorex, La Jolla), p. 25.

Pasteur, E. C., and R. Mulvaney, 2000, J. Geophys. Res. 105, 11525.

Paterson, W. S. B., 1994, The Physics of Glaciers, 3rd ed. (Elsevier, London).

Pengra, D. B., and J. G. Dash, 1992, J. Phys.: Condens. Matter 4, 7317.

Pengra, D. B., D.-M. Zhu, and J. G. Dash, 1991, Surf. Sci. 245, 125.

Peppiatt, S. J., and J. R. Sambles, 1975, Proc. R. Soc. London, Ser. A 345, 387.

Peppin, S. S. L., J. A. W. Elliott, and M. G. Worster, 2006, J. Fluid Mech. 554, 147.

Perutz, M. F., 1948, J. Glaciol. 1, 95.

Peter, T., 1999, in Ice Physics and the Natural Environment, J. S. Wettlaufer, J. G. Dash, and N. Untersteiner, NATO Advanced Studies Institute, No. 56, Series I: Global Environmental Change (Springer-Verlag, Heidelberg), pp. 143-68.

Peterson, R. A., and W. B. Krantz, 2003, J. Glaciol. 48, 69.

Petit, J. R., J. Jouzel, D. Raynaud, N. I. Barkov, J.-M. Barnola, I. Basile, M. Bender, J. Chappellaz, M. Davis, G. Delaygue, M. Delmotte, V. M. Kotlyakov, M. Legrand, V. Y. Lipenkov, C. Lorius, L. Pépin, C. Ritz, E. Saltzman, and M. Stievenard, 1999, Nature (London) 399, 429.

Phillips, J. M., 1989, Langmuir 5, 571.

Pittenger, B., D. J. Cook, C. R. Slaughterbeck, and S. C. Fain, Jr., 1998, J. Vac. Sci. Technol. A 16, 1832.

Pittenger, B., S. C. Fain, Jr., M. J. Cochran, J. M. K. Donev, B. E. Robertson, A. Szuchmacher, and R. M. Overney, 2001, Phys. Rev. B 63, 134102.
Plug, L. J., and B. T. Werner, 2001, J. Geophys. Res. 106, 8599. Plug, L. J., and B. T. Werner, 2002, Nature (London) 417, 929. Pluis, B., and A. W. denier van der Gon, 1988, in Chemistry and Physics of Solid Surfaces VII, edited by R. Vanselow and R. F. Howe (Springer-Verlag, Berlin).

Pluis, B., A. W. denier van der Gon, J. W. M. Frenken, and J. F. van der Veen, 1987, Phys. Rev. Lett. 59, 2678.

Pötschke, J., and V. Rogge, 1989, J. Cryst. Growth 94, 726.

Price, P. B., 2000, Proc. Natl. Acad. Sci. U.S.A. 97, 1247.

Radd, F. J., and D. H. Oertle, 1973, in Proceedings of the Second International Conference on Permafrost, Yakutsk, p. 377.

Rall, M., J. P. Brison, and N. S. Sullivan, 1991, Phys. Rev. B 44, 9639.

Rault, J., 2003, Eur. Phys. J. B 36, 627.

Raymond, C. F., 2000, J. Glaciol. 46, 665.

Raymond, C. F., and W. D. Harrison, 1975, J. Glaciol. 14, 213.

Rempel, A. W., 2000, Ph.D. thesis (Cambridge University, Cambridge, UK).

Rempel, A. W., 2005, Ann. Glaciol. 40, 191.

Rempel, A. W., E. D. Waddington, J. S. Wettlaufer, and M. G. Worster, 2001, Nature (London) 411, 568.

Rempel, A. W., and J. S. Wettlaufer, 2003a, J. Glaciol. 49, 397.

Rempel, A. W., and J. S. Wettlaufer, 2003b, Can. J. Phys. 81, 89.

Rempel, A. W., J. S. Wettlaufer, and E. D. Waddington, 2002, J. Geophys. Res. 107, 2330.

Rempel, A. W., J. S. Wettlaufer, and M. G. Worster, 2001, Phys. Rev. Lett. 87, 088501.

Rempel, A. W., J. S. Wettlaufer, and M. G. Worster, 2004, J. Fluid Mech. 498, 227.

Rempel, A. W., and M. G. Worster, 1999, J. Cryst. Growth 205, 427.

Rempel, A. W., and M. G. Worster, 2001, J. Cryst. Growth 223, 420.

Rice, J. M., 2006, J. Geophys. Res. 111, B05311.

Röthlisberger, H., 1972, J. Glaciol. 11, 205.

Roush, T. L., 2001, J. Geophys. Res. 106, 33315.

Sadtchenko, V., M. Brindza, M. Chonde, B. Palmore, and R. Eom, 2004, J. Chem. Phys. 121, 11980.

Sadtchenko, V., and G. Ewing, 2002, J. Chem. Phys. 116, 4686.

Sartor, J. D., 1981, J. Atmos. Sci. 38, 218.

Saunders, C. P. R., 1993, J. Appl. Meteorol. 32, 642.

Saunders, C. P. R., 1994, J. Geophys. Res. 99, 10773.

Scherer, G. W., 1993, J. Non-Cryst. Solids 155, 1.

Scherer, G. W., 1999, Cem. Concr. Res. 29, 1347.

Schick, M., 1990, in Introduction to Wetting Phenomena in Liquids at Interfaces, edited by J. Charvolin, J. F. Joanny, and J. Zinn-Justin, Les Houches Lectures Session XLVIII (Elsevier, New York).

Schick, M., and W. Shih, 1987, Phys. Rev. B 35, 5030.

Schoof, C., 2005, Proc. R. Soc. London, Ser. A 461, 609.

Schweizer, J., J. B. Jamieson, and M. Schneebeli, 2003, Rev. Geophys. 41, 2.

Seki, M., K. Kobayashi, and J. Nakahara, 1981, J. Phys. Soc. Jpn. 50, 2643.

Sen, S., B. K. Dhindaw, D. M. Stefanescu, A. Catalina, and P. A. Curreri, 1997, J. Cryst. Growth 173, 574.

Severinghaus, J. P., T. Sowers, E. J. Brook, R. B. Alley, and M. L. Bender, 1998, Nature (London) 391, 141.

Shangguan, D., S. Ahuja, and D. M. Stefanescu, 1992, Metall. Trans. A 23A, 669.

Shankland, T. J., R. J. O'Connell, and H. S. Waff, 1981, Rev. Geophys. Space Phys. 19, 394. 
Sharma, A., J. H. Scott, G. D. Cody, M. L. Fogel, R. H. Hazen, R. J. Hemley, and W. T. Huntress, 2002, Science 295, 1514.

Sherwood, S. C., V. T. J. Phillips, and J. S. Wettlaufer, 2006, Geophys. Res. Lett. 33, L05804.

Shih, W. H., Z. Q. Wang, X. C. Zeng, and D. Stroud, 1987, Phys. Rev. A 35, 2611.

Shimoda, M., T. Misuzaki, M. Hiroi, A. Hirai, and K. Eguchi, 1986, J. Low Temp. Phys. 64, 285.

Shreve, R. L., 1972, J. Glaciol. 11, 205.

Shreve, R. L., 1984, J. Glaciol. 30, 341.

Sletten, R. S., B. Hallet, and R. C. Fletcher, 2003, J. Geophys. Res. 108, 8044.

Slovak, J., 2001, Physica A 292, 87.

Smith, C. S., 1948, Trans. Am. Inst. Min., Metall. Pet. Eng. 175, 15.

Smith, M. W., and A. R. Tice, 1988, USA Cold Regions Research and Engineering Laboratory CRREL Report No. 8818.

Sokratov, S. A., 2001, Cold Regions Sci. Technol. 33, 263.

Solomon, S., 1988, Rev. Geophys. 26, 131.

Stern, L. A., W. B. Durham, and S. H. Kirby, 1997, J. Geophys. Res. 102, 5313.

Stranski, I. N., 1942, Naturwiss. 28, 30.

Stock, K. D., 1980, Surf. Sci. 91, 655.

Stow, C. D., and G. J. Turner, 1987, Philos. Mag. A 56, 783.

Style, R., and M. G. Worster, 2005, Phys. Rev. Lett. 95, 176102.

Sullivan, D. E., and M. M. Telo da Gama, 1988, in Fluid Interfacial Phenomena, edited by C. A. Croxton (Wiley, New York).

Sutton, A. P., and R. W. Balluffi, 1995, Interfaces in Crystalline Materials (Oxford University, New York).

Suzanne, J., and J. M. Gay, 1996, in Handbook of Surface Science, edited by W. N. Unertl (Elsevier, Amsterdam), Vol. 1.

Swainson, I. P., and E. M. Schulson, 2001, Cem. Concr. Res. 31, 1821.

Swainson, I. P., and E. M. Schulson, 2004, J. Geophys. Res. 109, B12205.

Tabazadeh, A., O. B. Toon, and E. J. Jensen, 1999, Geophys.

Res. Lett. 26, 221.

Taber, S., 1929, J. Geol. 37, 428.

Taber, S., 1930, J. Geol. 38, 303.

Takagi, M., 1954, J. Phys. Soc. Jpn. 9, 359.

Takahashi, T., 1969, J. Atmos. Sci. 26, 1253.

Takahashi, T., 1978, J. Atmos. Sci. 35, 1536.

Takashi, T., T. Ohrai, H. Yamamoto, and J. Okamoto, 1981, Eng. Geol. (Amsterdam) 18, 245.

Talamucci, F., 2003, Math. Comput. Modell. 37, 595.

Tammann, G., 1909, Z. Phys. Chem., Stoechiom. Verwandtschaftsl. 68, 257.

Tartaglino, U., T. Zykova-Timan, F. Ercolessi, and E. Tosatti, 2005, Phys. Rep. 411, 291.

Telford, J. W., and J. S. Turner, 1963, Philos. Mag. 8, 527.

Tell, J. L., and H. J. Maris, 1983, Phys. Rev. B 28, 5122.

Thompson, J. R., H. J. Kim, C. Cantoni, D. K. Christen, R. Feenstra, and D. T. Verebelyi, 2004, Phys. Rev. B 69, 104509. Thomson, E. S., J. S. Wettlaufer, and L. A. Wilen, 2005, Bull. Am. Phys. Soc. 50, 127.

Thomson, J., 1849, Trans. - R. Soc. Edinbrgh 16, 575.

Thomson, W., 1861, Proc. R. Soc. London 11, 198.

Thomson, W., 1871, Philos. Mag. 42, 448.

Thorsteinsson, Th., J. Kipfstuhl, and H. Miller, 1997, J. Geophys. Res. 102, 26583.

Townsend, D. W., and R. P. Vickery, 1967, Philos. Mag. 16,
1275 .

Tsionsky, V., D. Zagidulin, and E. Gileadi, 2002, J. Phys. Chem. 106, 13089.

Tulaczyk, S., W. B. Kamb, and H. F. Engelhardt, 2000, J. Geophys. Res. 105, 483.

Turner, G. J., and C. D. Stow, 1984, Philos. Mag. A 49, L25.

Turtle, E. P., and E. Pierazzo, 2001, Science 294, 1326.

Tyndall, J., 1856, Proc. R. Soc. London 9, 76.

Uhlmann, D. R., B. Chalmers, and K. A. Jackson, 1964, J. Appl. Phys. 35, 2986.

Untersteiner, N., 1968, J. Geophys. Res. 73, 1251.

Valdez, M. P., G. A. Dawson, and R. C. Bales, 1989, J. Geophys. Res. 94, 1095.

Valiullin, R., and I. Furo, 2002, J. Chem. Phys. 117, 2307.

van der Eerden, J. P., 1993, J. Cryst. Growth 128, 62.

van der Veen, J. F., B. Pluis, and A. W. Denier van der Gon, 1988, Chemistry and Physics of Solid Surfaces (SpringerVerlag, Berlin), Vol. VII, p. 455.

van Miltenburg, J. C., and J. P. van der Eerden, 1993, J. Cryst. Growth 128, 1143.

Verwey, E. J. W., and J. Th. G. Overbeek, 1948, Theory of Stability of Lyophobic Colloids (Elsevier, Amsterdam).

Viasnoff, V., F. Lequeux, and D. J. Pine, 2002, Rev. Sci. Instrum. 73, 2336.

Viklander, P., and E. Eigenbrod, 2000, Cold Regions Sci. Technol. 31, 151.

Vold, C. L., and M. E. Glicksman, 1972, in The Nature and Behavior of Grain Boundaries, edited by $\mathrm{H}$. Hu (Plenum, New York), p. 171.

Walder, J., and B. Hallet, 1985, Geol. Soc. Am. Bull. 96, 336.

Walder, J., and B. Hallet, 1986, Arct. Alp. Res. 18, 27.

Walford, M. E. R., and J. F. Nye, 1991, J. Glaciol. 37, 107.

Wang, H., R. C. Bell, M. J. Iedema, A. A. Tsekouras, and J. P. Cowin, 2005, Astrophys. J. 620, 1027.

Warren, S. G., and S. R. Hudson, 2003, Appl. Environ. Microbiol. 69, 6340.

Watanabe, K., and M. Mizoguchi, 2000, J. Cryst. Growth 213, 135.

Watanabe, K., and M. Mizoguchi, 2001, Cold Regions Sci. Technol. 34, 103.

Watanabe, K., Y. Muto, and M. Mizoguchi, 2001, Cryst. Growth Des. 1, 207.

Weber, T. A., and F. H. Stillinger, 1983, J. Phys. Chem. 87, 4277.

Website: www.mysteries of canada.com/Alberta/habbakuk.htm Weeks, W. F., 1998, in Physics of Ice-Covered Seas, edited by M. Leppäranta (University of Helsinki Press, Helsinki), pp. 25-104.

Weeks, J. D., and G. H. Gilmer, 1979, Adv. Chem. Phys. 40, 157.

Weertman, J., 1957, J. Glaciol. 3, 33.

Weertman, J., 1983, Annu. Rev. Earth Planet Sci. 11, 215.

Wei, X., P. B. Miranda, C. Zhang, and Y. R. Shen, 2002, Phys. Rev. B 66, 085401.

Wettlaufer, J. S., 1998, in Physics of Ice-Covered Seas, edited by M. Leppäranta (University of Helsinki Press, Helsinki), pp. 105-194.

Wettlaufer, J. S., 1999a, Phys. Rev. Lett. 82, 2516.

Wettlaufer, J. S., 1999b, Philos. Trans. R. Soc. London, Ser. A 357, 3403.

Wettlaufer, J. S., 2001, Interface Sci. 9, 115.

Wettlaufer, J. S., M. Jackson, and M. Elbaum, 1994, J. Phys. A 27, 5957. 
Wettlaufer, J. S., and M. G. Worster, 1995, Phys. Rev. E 51, 4679 .

Wettlaufer, J. S., and M. G. Worster, 2006, Annu. Rev. Fluid Mech. 38, 427.

Wettlaufer, J. S., M. G. Worster, and H. E. Huppert, 1997, J. Fluid Mech. 344, 291.

Wettlaufer, J. S., M. G. Worster, and H. E. Huppert, 2000, J. Geophys. Res. 105, 1123.

Wettlaufer, J. S., M. G. Worster, L. A. Wilen, and J. G. Dash, 1996, Phys. Rev. Lett. 76, 3602.

Wilen, L. A., and J. G. Dash, 1995a, Phys. Rev. Lett. 74, 5076.

Wilen, L. A., and J. G. Dash, 1995b, Science 270, 184.

Wilen, L. A., J. S. Wettlaufer, M. Elbaum, and M. Schick, 1995, Phys. Rev. B 52, 12426.

Williams, E. R., R. Zhang, and R. Rydock, 1991, J. Atmos. Sci. 48, 2195.

Wolff, E. W., 1996, in Chemical Exchange between the Atmosphere and Polar Ice, edited by E. W. Wolff and R. C. Bales, NATO Advanced Studies Institute No. 53, Series I: Global Environmental Change (Springer, Berlin), p. 541.
Wolff, E. W., and R. Mulvaney, 1988, Ann. Glaciol. 11, 194. Wolff, E. W., and J. G. Paren, 1984, J. Geophys. Res. 89, 9433. Workman, E. J., and S. E. Reynolds, 1948, Phys. Rev. 74, 709. Worster, M. G., 1997, Annu. Rev. Fluid Mech. 29, 91.

Worster, M. G., and J. S. Wettlaufer, 1999, in Fluid Dynamics at Interfaces, edited by W. Shyy and R. Narayanan (Cambridge University Press, Cambridge, England), p. 339.

Wronski, C. R. M., 1967, Br. J. Appl. Phys. 18, 1931.

Wulff, G., 1901, Z. Kristallogr. 34, 449.

Zhang, T., J. A. Heginbottom, R. G. Barry, and J. Brown, 2000, Polar Geogr. 24, 126.

Zhang, R., M.-T. Leu, and M. Molina, 1996, Geophys. Res. Lett. 23, 1669.

Zhu, D.-M., 2000, Phys. Rev. E 63, 012502.

Zhu, D.-M., and J. G. Dash, 1986, Phys. Rev. Lett. 57, 2959.

Zhu, D.-M., and J. G. Dash, 1988, Phys. Rev. Lett. 60, 432.

Zhu, D.-M., O. E. Vilches, J. G. Dash, B. Sing, and J. S. Wettlaufer, 2000, Phys. Rev. Lett. 85, 4908.

Zwally, H. J., W. Abdalati, T. Herring, K. Larson, J. Saba, and K. Steffan, 2002, Science 297, 218. 